University of Rhode Island

DigitalCommons@URI

Open Access Dissertations

2021

\title{
MULTI-VIEW ROBUST REPRESENTATION LEARNING
}

Lusi Li

University of Rhode Island, lusi_li@uri.edu

Follow this and additional works at: https://digitalcommons.uri.edu/oa_diss

\section{Recommended Citation}

Li, Lusi, "MULTI-VIEW ROBUST REPRESENTATION LEARNING" (2021). Open Access Dissertations. Paper 1249.

https://digitalcommons.uri.edu/oa_diss/1249

This Dissertation is brought to you for free and open access by DigitalCommons@URI. It has been accepted for inclusion in Open Access Dissertations by an authorized administrator of DigitalCommons@URI. For more information, please contact digitalcommons-group@uri.edu. 
MULTI-VIEW ROBUST REPRESENTATION LEARNING

BY

LUSI LI

A DISSERTATION SUBMITTED IN PARTIAL FULFILLMENT OF THE REQUIREMENTS FOR THE DEGREE OF

DOCTOR OF PHILOSOPHY

IN

ELECTRICAL ENGINEERING

UNIVERSITY OF RHODE ISLAND

2021 
DOCTOR OF PHILOSOPHY DISSERTATION

$\mathrm{OF}$

LUSI LI

APPROVED:

Dissertation Committee:

$\begin{array}{ll}\text { Major Professor } & \text { Haibo He } \\ & \text { Yan (Lindsay) Sun } \\ & \text { Lisa DiPippo } \\ & \text { Brenton DeBoef } \\ & \text { DEAN OF THE GRADUATE SCHOOL }\end{array}$

UNIVERSITY OF RHODE ISLAND

2021 


\begin{abstract}
With the widespread deployment of sensors and the Internet-of-Things, multiview data have become more common and publicly available. For example, a self-driving car uses radar, lidar, and camera sensors to collect real-time 3D information to drive safely on the road; disease diagnosis models utilize multiple modalities of neuroimage data, clinical scores, and genetics measurements for disease prediction; object detection techniques prefer object images from different views for high-fidelity recognition. The presence of multiple information sources provides an opportunity of learning better representations to improve performance by analyzing multiple views simultaneously and also poses great challenges for the existing data representation algorithms. First, different views tend to be treated as different domains from different distributions due to the view discrepancy. Second, they often require large-scale labeled data to sufficiently learn such representations, which significantly hinders their adaption into unsupervised learning tasks, and limits their applications into critical domains where obtaining massive labeled data is prohibitively expensive. To enable learning on those domains, this dissertation focuses on robust representation learning-based algorithms to alleviate the view discrepancy of the multi-view data in an unsupervised manner.

Specially, we explore two scenarios upon data association for robust representation learning of multi-view data: First, the samples across different views have a sample-wise association in multi-view data, falling in the multi-view clustering scenario; Second, the samples across different views have a class-wise association, falling in the unsupervised domain adaption scenario, where the discriminant knowledge (representations) of views with labeled data samples are transferred to the views with unlabeled data samples.
\end{abstract}




\section{ACKNOWLEDGMENTS}

I would like to first express my deepest appreciation to my advisor, Prof. Haibo He, for supporting me during the past four years. Prof. He is someone you will never forget once you meet him. He has a great research vision and always steered me in the right direction whenever I needed it. He patiently instructed and encouraged me to think big and not to give up when all seemed not to be working out. My current achievements would not have been possible without his persistent help and dedicated involvement at every stage.

Besides my advisor, I would also like to show gratitude to the rest of my committee members: Prof. Yan (Lindsay) Sun, Prof. Lisa DiPippo, and Prof. Marco Alvarez, for their encouragement and insightful comments. I am grateful for the invaluable support and assistance that they all provided throughout the preparation of my research plan, proposal, dissertation, and defense.

My sincere thanks also go to Prof. Richard J. Vaccaro, Prof. Zhen Ni, Prof. Xiangnan Zhong, Prof. Jun Yan, Prof. Bo Tang, Prof. Yufei Tang, Prof. Fanrong Wei, Prof. Zhengming Ding, Dr. Zhiqiang Wan, and Dr. He Jiang for sharing expertise and valuable guidance on my faculty job searching.

I thank my fellow labmates in the Computational Intelligence and SelfAdaptive System (CISA) Laboratory: Yazhou Zhang, Zhenhua Wang, and Hepeng Li for all the unconditional support and the fun we have had. I am grateful to all visiting scholars and friends for all your help and for the great moments that we had here. I deeply thank my parents and brother for their timely encouragement.

I would like to extend my sincere thanks to the support for my Ph.D. research from the National Science Foundation (NSF) under grants CNS 1439011, ECCS 1917275, and ECCS 1731672. Their support provided me the opportunity to explore the important research topics during my Ph.D study. 


\section{TABLE OF CONTENTS}

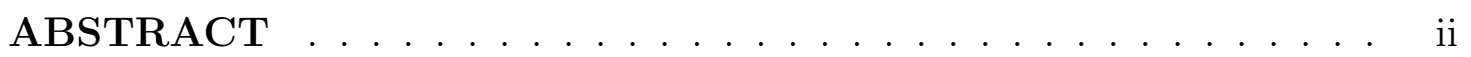

ACKNOWLEDGMENTS ................... . . iii

TABLE OF CONTENTS $\ldots \ldots \ldots \ldots \ldots \ldots$ iv

LIST OF FIGURES $\ldots \ldots \ldots \ldots \ldots \ldots \ldots$ viii

LIST OF TABLES . . . . . . . . . . . . . . . . xi

\section{CHAPTER}

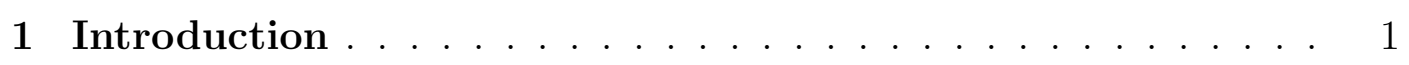

1.1 Research Motivations and Challenges ............ 1

1.2 Multi-view Clustering . . . . . . . . . . . . . . . . . 2

1.3 Unsupervised Domain Adaptation . . . . . . . . . . . . . . . 4

1.4 Dissertation Outline . . . . . . . . . . . . . . . . . 5

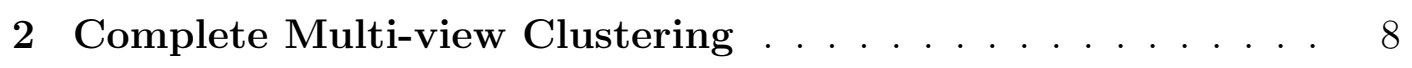

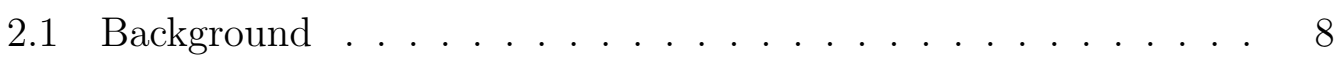

2.2 Related Work . . . . . . . . . . . . . . . . . . . . . 11

2.3 Proposed Method . . . . . . . . . . . . . . . . . . . . 12

2.3 .1 View Graph Learning . . . . . . . . . . . . . . . . . 12

2.3.2 Unified Graph Learning . . . . . . . . . . . . . . 13

2.3 .3 Consensus Anchor Learning . . . . . . . . . . . . 15

2.3.4 Optimal Bipartite Graph Learning . . . . . . . . . 16

2.4 Optimization Strategy $\ldots \ldots \ldots \ldots$ 


\section{Page}

2.4.1 Fix $\left\{\delta_{v}\right\}_{v=1}^{m}, U, F$, and $\left\{A_{v}\right\}_{v=1}^{m}$, Update $\{S\}_{v=1}^{m} \ldots \ldots 18$

2.4.2 Fix $\{S\}_{v=1}^{m}, U, F$, and $\left\{A_{v}\right\}_{v=1}^{m}$, Update $\left\{\delta_{v}\right\}_{v=1}^{m} \ldots \ldots$

2.4.3 Fix $\{S\}_{v=1}^{m},\left\{\delta_{v}\right\}_{v=1}^{m}, F$, and $\left\{A_{v}\right\}_{v=1}^{m}$, Update $U \ldots 20$

2.4.4 Fix $\{S\}_{v=1}^{m},\left\{\delta_{v}\right\}_{v=1}^{m}, U$, and $\left\{A_{v}\right\}_{v=1}^{m}$, Update $F \ldots 22$

2.4.5 Fix $\{S\}_{v=1}^{m},\left\{\delta_{v}\right\}_{v=1}^{m}, U$, and $F$, Update $\left\{A_{v}\right\}_{v=1}^{m} \ldots .22$

2.5 Complexity and Convergence Analysis . . . . . . . . . . 23

2.5 .1 Complexity Analysis . . . . . . . . . . . . . 23

2.5.2 Convergence Analysis . . . . . . . . . . . . . . . . 24

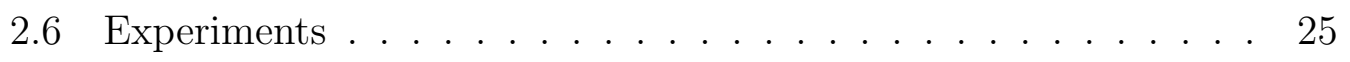

2.6.1 Experiments on Synthetic Datasets . . . . . . . . 26

2.6.2 Experiments on Real-world Datasets . . . . . . . . . 28

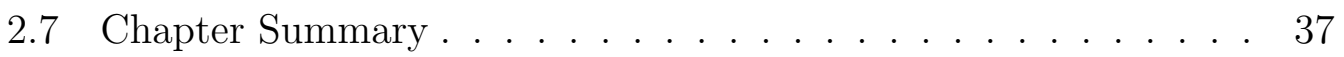

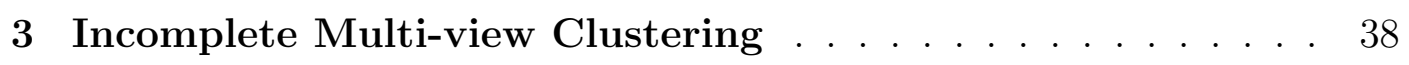

3.1 Background $\ldots \ldots \ldots \ldots \ldots \ldots \ldots$

3.2 Related Work . . . . . . . . . . . . . . . . . . . . 41

3.3 Proposed Method . . . . . . . . . . . . . . . . . . . . . 43

3.3.1 Adaptive Base Partition Learning . . . . . . . . . . . . 43

3.3.2 Multi-View Partition Fusion . . . . . . . . . . . . . 46

3.3.3 Consensus Graph Construction . . . . . . . . . . 47

3.4 Optimization Strategy $\ldots \ldots \ldots$. . . . . . . . . . . . 49

3.4.1 Fix $\left\{F^{v}\right\}_{v=1}^{m}, H,\left\{w_{v}\right\}_{v=1}^{m}, S$, and $Q$, Update $\left\{A^{v}\right\}_{v=1}^{m} \ldots 49$

3.4.2 Fix $\left\{A^{v}\right\}_{v=1}^{m}, H,\left\{w_{v}\right\}_{v=1}^{m}, S$, and $Q$, Update $\left\{F^{v}\right\}_{v=1}^{m} \ldots 50$

3.4.3 Fix $\left\{A^{v}\right\}_{v=1}^{m},\left\{F^{v}\right\}_{v=1}^{m},\left\{w_{v}\right\}_{v=1}^{m}, S$, and $Q$, Update $H \ldots 51$ 


\section{Page}

3.4.4 Fix $\left\{A^{v}\right\}_{v=1}^{m},\left\{F^{v}\right\}_{v=1}^{m}, H, S$, and $Q$, Update $\left\{w_{v}\right\}_{v=1}^{m} \ldots 52$

3.4.5 Fix $\left\{A^{v}\right\}_{v=1}^{m},\left\{F^{v}\right\}_{v=1}^{m}, H,\left\{w_{v}\right\}_{v=1}^{m}$, and $Q$, Update $S \ldots 52$

3.4.6 Fix $\left\{A^{v}\right\}_{v=1}^{m},\left\{F^{v}\right\}_{v=1}^{m}, H,\left\{w_{v}\right\}_{v=1}^{m}$, and $S$, Update $Q \ldots 53$

3.4.7 Determine the Values of Parameters Adaptively . . . . . 54

3.5 Experiments ........................ 56

3.5.1 Experimental Settings . . . . . . . . . . . . . 56

3.5.2 Results and Analysis . . . . . . . . . . . . . . 58

3.6 Chapter Summary . . . . . . . . . . . . . . . . . . . . . . . 64

4 Unsupervised Domain Adaptation . . . . . . . . . . . . 65

4.1 Background . . . . . . . . . . . . . . . 65

4.2 Related Work . . . . . . . . . . . . . . . . . . . 67

4.2 .1 Domain Adaptation . . . . . . . . . . . . . . . . 67

4.2 .2 Discrepancy Metric . . . . . . . . . . . . . . . . . . 68

4.3 Proposed Method . . . . . . . . . . . . . . . . . . . . . . 69

4.3 .1 Feature Learning . . . . . . . . . . . . . . . . 70

4.3.2 Category-Invariant Representation Learning . . . . . . 70

4.3 .3 Overall Objective Function . . . . . . . . . . . . . . 74

4.4 Experiments . . . . . . . . . . . . . . . . 75

4.4.1 Experimental Settings _. . . . . . . . . . . 75

4.4 .2 Results and Analysis . . . . . . . . . . . . . . 77

4.5 Chapter Summary . . . . . . . . . . . . . . . . . . . . . 80

5 Unsupervised Partial Domain Adaptation . . . . . . . . . . 81

5.1 Background . . . . . . . . . . . . . . 81 


\section{Page}

5.2 Related Work . . . . . . . . . . . . . . . . . . 84

5.2 .1 Partial Domain Adaptation . . . . . . . . . . . 84

5.2 .2 Discrepancy Metric . . . . . . . . . . . . . . . . . 87

5.3 Proposed Method . . . . . . . . . . . . . . . . . . . . 87

$5.3 .1 \quad$ Feature Extractor . . . . . . . . . . . . . . . . . . 89

5.3 .2 Reweighting Network . . . . . . . . . . . . . . . . . . 89

5.3 .3 Dual Alignment Network . . . . . . . . . . . . . . . . 92

5.3.4 Overall Objective Function . . . . . . . . . . . . . . . 95

5.4 Experiments . . . . . . . . . . . . . . . . . 96

5.4 .1 Experimental Settings _. . . . . . . . . . . . . . 96

5.4 .2 Results and Analysis . . . . . . . . . . . . . . . 100

5.5 Chapter Summary . . . . . . . . . . . . . . . . . . . . . 110

6 Conclusion and Future Work . . . . . . . . . . . . . 112

6.1 Conclusion . . . . . . . . . . . . . . . . . . . . 112

6.2 Future Work . . . . . . . . . . . . . . . . . . . . . . . 113

LIST OF REFERENCES . . . . . . . . . . . . . . . . . . . . 115

BIBLIOGRAPHY . . . . . . . . . . . . . . . . . . . . . . 124 


\section{LIST OF FIGURES}

Figure

Page

1 An overview of the dissertation organization. . . . . . . . . 6

2 The framework of our proposed BIGMC method. . . . . . . . . 9

3 Clustering results on Two-Moon data set. The upper row includes the original first view data, the learned graph with the learned $S^{1}$, and the learned graph with the learned $U$. The lower row contains the original second view data, the learned graph with the learned $S^{2}$, and the learned graph with the learned $U$. The red dots are cluster 1 , and the blue dots are cluster 2 . The pink squares are the learned anchor points, and the green lines are the learned connections between data points and anchor points. .................... 27

4 Clustering results on Three-Circle data set. The upper row includes the original first view data, the learned graph with the learned $S^{1}$, and the learned graph with the learned $U$. The lower row contains the original second view data, the learned graph with the learned $S^{2}$, and the learned graph with the learned $U$. The red, blue, and black dots are cluster 1 , cluster 2 , and cluster 3 . The pink squares are the learned anchor points, and the green lines are the learned connections between data points and anchor points. . . . . . . . . . . . . . . . . . . . . 28

5 The learning results of anchors by BIGMC on 3 real-world datasets compared with the ground truth and the predicted number of samples in each class. (a) 3sources; (b) Caltech-7;

(c) WebKB. . . . . . . . . . . . . . . . . . . 34

Clustering performance comparison of BIGMC and its 4 variants on 7 real-world datasets in terms of metrics (ACC, NMI, and F-M). . . . . . . . . . . . . . . . . 35

$7 \quad$ Convergence curves over different datasets. (a) 100leaves. (b) Caltech-20. (c) WebKB. (d) YaleB. . . . . . . . . . . . . . 36

8 The framework of our proposed JPG method. . . . . . . . . . . 40 
$9 \quad$ Clustering performance results with different PER settings in the second case: (a)-(c) on 100Leaves dataset; (d)-(f) on ORL dataset. . . . . . . . . . . . . . 6 60

10 Clustering performance results with different PER settings in the second case: (a)-(c) on Caltech101-7 dataset; (d)-(f) on Caltech101-20 dataset. . . . . . . . . . . . . . . . 61

11 Average clustering performance results with different PER settings in the second case: on (a) 100Leaves dataset; (b) on ORL dataset; (c) on Caltech101-7 dataset; (d) on Caltech101-20 dataset. . . . . . . . . . . . . . . 62

12 Convergence curves with 60\% PER settings on (a) 100Leaves data set; (b) 3Sources data set; (c) ORL data set. . . . . . . . . 64

13 The framework of our proposed ADACT method. . . . . . . . . 66

14 The t-SNE visualization of learned features for $\mathrm{A} \rightarrow \mathrm{W}$ task on Office-Caltech dataset by MMD, DANN, CORAL, WDGRL, and ADACT. The top row shows domain comparisons, where blue triangle and orange square points denote source and target domains, respectively. The bottom row shows category discrimination, where triangle and square points represent source and target domains, and points are colored w.r.t. their classes. . . . 79

15 The framework of our proposed DAPDA method. . . . . . . . . 81

16 Illustration of several image examples for (a) Office-31 dataset, (b) Office-Caltech dataset, and (c) Office-Home dataset. . . . . 97

17 The t-SNE visualization of learned features of compared and proposed methods for A10 $\rightarrow$ C5 task on Office-Caltech dataset. The blue, green, and orange dots represent the source shared samples, source outlier samples, and target samples. The orange dots are expected to be aligned with the blue dots. . . . . . . . 104

18 The t-SNE visualization of learned features of compared and proposed methods for A10 $\rightarrow$ C5 task on Office-Caltech dataset. Each color represents a class. The dots with the same color are expected to be aligned. . . . . . . . . . . . . . . . 105

19 Parameter sensitivity on the task W31 $\rightarrow$ A10, A10 $\rightarrow$ C5, Ar65 $\rightarrow$ Pr25, and Pr65 $\rightarrow$ Cl25. (a) parameter $\alpha ;(b)$ parameter $\beta$. . 106 
20 For the task A10 $\rightarrow$ C5, (a) Loss and accuracy values w.r.t. number of iterations (x10); (b) Loss values w.r.t. number of iterations $(\mathrm{x} 10) \ldots \ldots \ldots$. . . . . . . . . . . . 107

21 For the task A31 $\rightarrow$ W10, histograms of class weights learned by (a) ResNet-50; (b) DANN; (c) PADA; (d) DAPDA. . . . . . 108

22 The accuracy curve of varying the number of target classes for $\mathrm{A} 31 \rightarrow \mathrm{W} 10$ task using AlexNet as the baseline. . . . . . . . . . . 108

23 The t-SNE visualization of learned features of compared and proposed methods for $\mathrm{A} \rightarrow \mathrm{C}$ task on Office-Caltech dataset. The blue and orange dots represent source and target samples, respectively. The orange dots are expected to be aligned with the blue dots. . . . . . . . . . . . . . . . . . . . . . . 109

24 The t-SNE visualization of learned features of compared and proposed methods for $\mathrm{A} \rightarrow \mathrm{C}$ task on Office-Caltech dataset. Each color represents a class. The dots with the same color are expected to be aligned. . . . . . . . . . . . . . . . . . 110 


\section{LIST OF TABLES}

Table

Page

1 Statistics of experimental data sets . . . . . . . . . . . . 29

$2 \quad$ Clustering results (mean \pm standard-deviation) with metrics (ACC, NMI, ARI, F-M, PRE, and REC) by different methods on 7 real-world datasets . . . . . . . . . . . . . 32

$3 \quad$ Averaged running time by different methods on 7 real-world datasets (in second) . . . . . . . . . . . . . 37

$4 \quad$ Statistics of experimental data sets . . . . . . . . . . . 57

$5 \quad$ Clustering performance results on three natural incomplete multi-view datasets in the first case . . . . . . . . . . . . . . 59

$6 \quad$ Clustering performance results on three incomplete multi-view datasets in the third case . . . . . . . . . . . 62

$7 \quad$ Average accuracy (\%) obtained on digits datasets . . . . . . . 77

8 Best accuracy (\%) obtained on Office-Caltech dataset with $\mathrm{DeCAF}_{7}$ features . . . . . . . . . . . . 78

$9 \quad$ Main notations and definitions in the proposed DAPDA method 88

10 Average accuracy (\%) of partial domain adaptation tasks on the Office-31 dataset (AlexNet) . . . . . . . . . . . . . 101

11 Average accuracy (\%) of partial domain adaptation tasks on Office-Caltech dataset (AlexNet) . . . . . . . . . . . 101

12 Average accuracy (\%) of partial domain adaptation tasks on Office-31 dataset (ResNet-50) . . . . . . . . . . . 102

13 Average accuracy (\%) of partial domain adaptation tasks on Office-Home dataset (ResNet-50) . . . . . . . . . . . . . . 103

14 Average accuracy (\%) of full domain adaptation tasks on OfficeCaltech dataset (AlexNet) . . . . . . . . . . . . . . 109 


\section{CHAPTER 1}

\section{Introduction}

\section{$1.1 \quad$ Research Motivations and Challenges}

In the information age, the amount of data is exploding exponentially. Multiview data are extensively accessible due to multiple information sources, which

facilitates learning better representations in many real-world applications [1]. In multi-view data, samples can be described by multi-modal measurements of an underlying signal (e.g., text+image, video+audio, audio+articulation, and text in different languages), or synthetic views of the uni-modal measurements (e.g., word + context words, different time stamps of a time sequence, and web text+text of inbound hyperlinks) $[2,3]$.

Multi-view representation learning algorithms exploit this point to learn more information than those of single-view data analysis methods. Recent breakthroughs of them have a wide spectrum of application domains, such as pattern recognition, natural language processing, medical diagnosis, and wireless communications. This demonstrates the power of representation learning in extracting valuable information, especially from heterogeneous multi-view data. However, different views tend to be regarded as different domains from different distributions due to the large view discrepancy and heterogeneity. Therefore, the first challenge is how to reduce the view discrepancy by learning robust representations, especially when facing specific problems by either fusing the representations across multiple views or adapting the representations from some views to others.

The algorithms of learning representations from multi-view data have achieved some successes in the supervised manner. However, they often require large-scale labeled data to successfully learn such representations, which significantly hinders their adaption into unsupervised learning tasks, and limits their applications into 
critical domains where obtaining massive labeled data is prohibitively expensive. The second challenge is how to enable learning on those domains in the unsupervised manner.

In this dissertation, we focus on two scenarios upon data association for learning robust representations from multi-view data: First, the samples across different views have sample-wise association in multi-view data, falling in the multiview clustering scenario; Second, the samples across different views have classwise association, falling in the unsupervised domain adaption scenario, where the discriminant knowledge (representations) of views with labeled data samples are transferred to the views with unlabeled data samples. From the representation perspective, the two scenarios can also be treated as representation fusion and representation transfer, respectively. Multi-view clustering aims to learn representation from each view to boost clustering performance, and unsupervised domain adaption tends to transfer the representation from labeled source views to unlabeled target views. Hence, this dissertation presents robust representation learning-based solutions for these two scenarios. Multi-view clustering and unsupervised domain adaptation are introduced next.

\subsection{Multi-view Clustering}

Clustering has long been serving as a critical unsupervised technique in pattern recognition, data mining, and machine learning. The aim of clustering is to group data objects into clusters such that data objects in the same cluster are more similar than those in different clusters. However, most existing clustering methods are concerned about single-view learning [2]. As Internet and communication technologies develop rapidly, many real-world data can be extracted from multiple

sources $[4,5]$, which makes it possible to produce multi-view data. In multi-view data, each object is associated with much richer information [6]. How to make full 
use of the information contained in multiple views to improve clustering results is referred to as be multi-view clustering [7].

Obviously, each view has its biases. If the multi-view clustering algorithms cannot explore valuable information and cope appropriately with multiple views, the clustering performance may be poorer than that by single-view clustering methods [8]. Thus, compared with single-view clustering, multi-view clustering is expected to achieve more robust and precise clustering results via exploiting the complementary information in multiple views [9]. Three main challenges need to overcome. The first one is how to extract valuable information from multiple views [10]. The second one is how to integrate the extracted information effectively $[11,12]$. The third one is how to learn the importance of each view for the clustering task [13]. Note that these three issues should be figured out simultaneously for multi-view clustering.

Numerous multi-view clustering approaches have been proposed, and can be roughly grouped into four classes based on different learning strategies: co-training learning [14], kernel learning [10, 15], subspace learning [16, 17], and spectral learning $[18,19,20]$. Thereinto, the co-training learning tries to produce a learner for each view by using the learned knowledge from one another view and thus the partitions of different views can be learned. Kernel learning aims to use one kernel or combine multiple kernels in a linearly or non-linearly manner to perform clustering, where a base kernel is pre-given for each view. The subspace learning attempts to find a common latent representation for all views for constructing a similarity matrix and then perform a spectral clustering strategy to obtain final results. While spectral learning $[13,21]$ aims to fuse low-dimensional embedding representations of different views into a unified representation, and then perform $K$-means method on the unified representation to generate the final clusters. Gen- 
erally, co-training-based approaches depend on the conditional independence of multiple views. The differences among multiple views are ignored. Subspacebased algorithms are sensitive to the quality of original feature representations. That is to say, they cannot find the underlying representation of the data with outliers. Additionally, multi-kernel-based methods are sensitive to the selection of base kernels. Most spectral-based methods need an additional clustering step to generate the final clusters.

In addition, a more challenging problem is incomplete multi-view clustering. The assumption of complete multi-view clustering is that each view of a sample exists. It does not always hold since some views of a sample are missing due to machine down or sensor fault. We call such data as incomplete multi-view data. Complete multi-view clustering methods cannot provide encouraging performance on incomplete multi-view data. Thus, incomplete multi-view clustering needs not only to make full use of the complementary information of all views, but also to eliminate the negative impacts of missing samples. To deal with incomplete views, incomplete multi-view clustering methods employ an imputation technique to fill the incomplete views or directly learn a common representation or subspace without imputation.

\subsection{Unsupervised Domain Adaptation}

Domain adaptation (DA) attempts to transfer knowledge from labeled source domains to facilitate the learning burden in the target domains with sparsely or no labeled samples. According to the availability of labeled target data, there are two categories of DA: semi-supervised DA and unsupervised DA. In the target domain, the former requires a number of labeled training samples, while the latter only needs unlabeled samples. Similarly, both of them call for a mass of unlabeled target domain data. Unsupervised domain adaptation (UDA) is our focus due to 
the challenge and practicality.

Many UDA methods attempt to train a classifier utilizing source samples to help target samples learning. However, the source domain and target domain are related but separated by different data distributions, resulting in weak generalization ability of models on target data. One effective strategy is to estimate important weights of source samples related to target samples such that the cross-domain discrepancy can be minimized [22]. Another successful strategy bridges different domains by learning domain-invariant features to reduce data distribution difference $[23,24]$. Recent studies have shown that more transferable representative features can be learned by deep neural networks [25, 26]. Extracting domain-invariant representations by embedding UDA in the pipeline of deep representation learning has achieved certain latest advances [23, 27, 28, 29].

Partial domain adaptation (PDA), as a more practical and challenging problem, assumes the target label space is subsumed into the source label space. In an unsupervised scenario, the target domain only has non-labeled data and the shared label space across domains is unknown. Thus PDA has another technical challenge: how to alleviate the negative transfer caused by the outlier source classes. Recently, there are four related methods including the importance weighted adversarial nets (IWAN) [30], selective adversarial network (SAN) [31], partial adversarial domain adaptation (PADA) [32], and Example Transfer Network (ETN) [33]. They have some successes in addressing the PDA by weighing each sample in the domainadversarial networks and matching either marginal or conditional distributions to align the source domain as well as the target domain.

\subsection{Dissertation Outline}

Given the challenges residing in multi-view data and inspired by the recent successes achieved by representation learning models, this dissertation focuses on 
multi-view robust representation learning including the following objectives: complete multi-view clustering, incomplete multi-view clustering, unsupervised domain adaptation, and unsupervised partial domain adaptation. The general organization of the rest parts of this dissertation is shown in Figure 1.

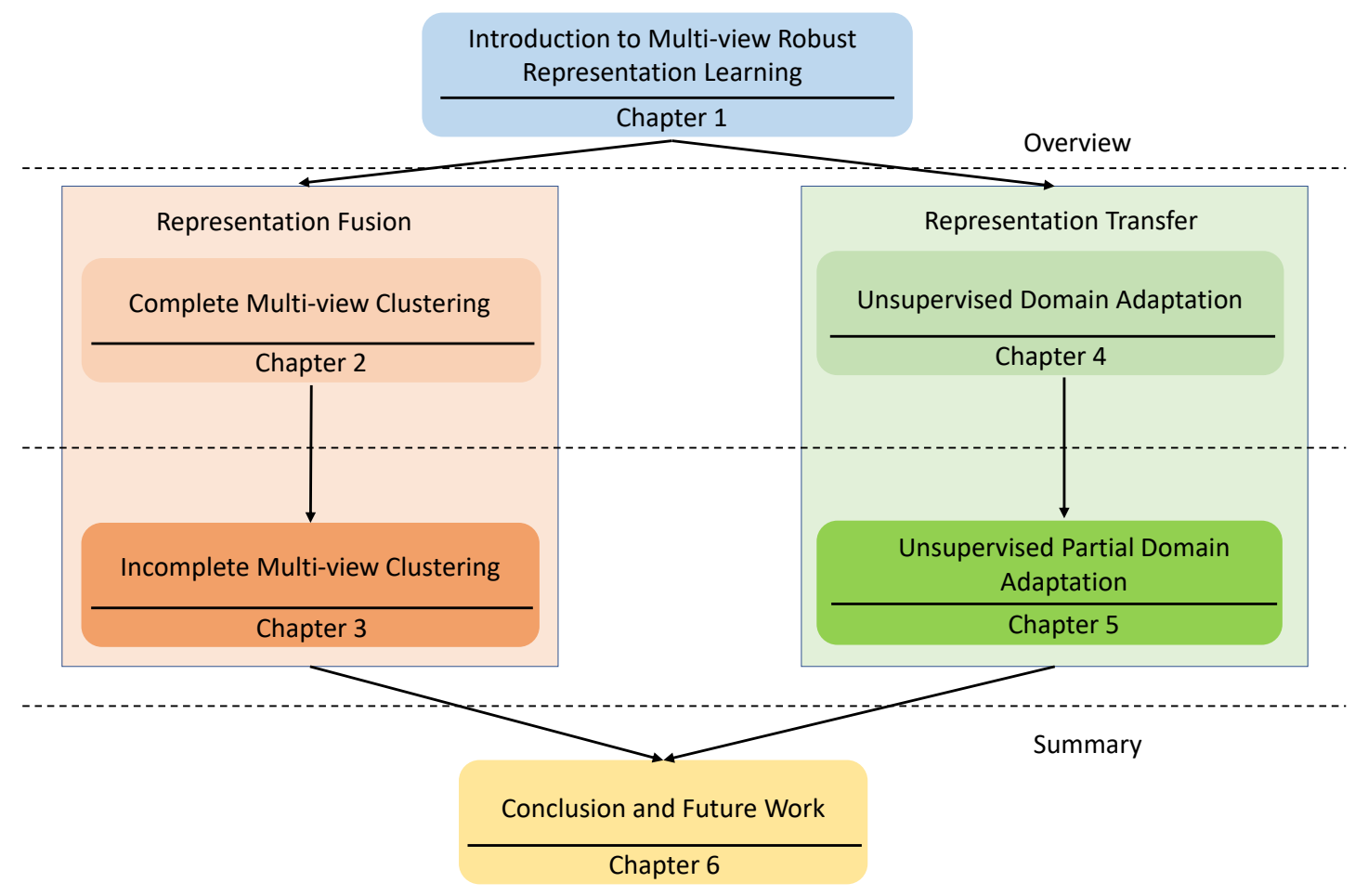

Figure 1. An overview of the dissertation organization.

Specifically, Chapter 2 presents a multi-view clustering framework based on bipartite graphs to capture the consensus representations. This framework can construct bipartite graphs between data points and a small number of representative uniform anchor points for different views. Moreover, this framework fuses those bipartite graphs to produce a unified graph including the required number of clusters.

Chapter 3 studies a joint graph learning model for incomplete multi-view clustering. This model can jointly construct local incomplete graphs, generate 
incomplete base partitions, stretch them to produce a unified partition, and employ it to learn a consensus graph. Specifically, this model can address both complete and incomplete multi-view clustering problems.

Chapter 4 develops an unsupervised domain adaptation approach by category transfer. This approach can estimate the domain discrepancies between source and target feature representations of the same category. Moreover, this approach should minimize those discrepancies to learn category-invariant feature representations and obtain optimal predicted target labels.

Chapter 5 develops a dual alignment network for partial domain adaptation. This network can exact source and target feature representations and reweight them by exploring the role of each sample. Moreover, this network can match the marginal distributions together with the conditional distributions, which can better determine the outlier source classes and align source and target domains. Chapter 6 summarizes the dissertation and discusses future work. 


\section{CHAPTER 2}

\section{Complete Multi-view Clustering}

\subsection{Background}

Among existing studies of multi-view clustering methods, graph-based methods are representative $[34,35]$. The structures of graphs consist of sets of vertexes and weighted edges among them. The similarity between any two vertexes is represented by the weight associated with the edge that connects them. Hence, graphs can effectively express the relationships among various types of data objects [36]. In graphs, each vertex corresponds to one data object and each weighted edge represents the similarity between two objects it connects.

In practice, the similarity relationships are expressed differently in different views [37]. Graph-based multi-view clustering methods aim to encode the similarity relationships among the data objects in the form of a unified graph matrix by combining the graph matrices of all views [38]. For the unified graph matrix, each non-zero element indicates the complementary similarity between two data objects. The final clusters are formed by employing an additional clustering method on the unified graph matrix. The clustering performance depends on the quality of each view graph and the fusion strategy. Although they have achieved some successes, there still exist several limitations. First, the consensus information of different views is not considered when learning each view graph matrix. Most existing methods learn pairwise similarities between objects for each view independently. This often leads to that the involved biases affect the quality of each view graph matrix. Motivated by [39], our method captures the consensus information by learning a small number of representative uniform anchor points for different views. Each anchor point is the centroid of the corresponding sub-cluster. That is to say, each view has an anchor set and these anchor points in different views preserve the 
information within the same sub-clusters. Second, they keep both the pre-given anchor set and the learned view graph matrix fixed in the fusion process (e.g., [39]). In this case, they are sensitive to the initialization and easy to trap in the local optimum. Our method learns each view bipartite graph matrix, the unified graph matrix, and uniform anchor points jointly in a mutually reinforcing way. Each of them can help the learning of the others. Third, most of them cannot adaptively learn the weight of each view without an additive hyper-parameter. The optimal value of the additive hyper-parameter needs to search in a large range [40]. Our method can determine an optimal weight for each view adaptively based on the corresponding learned view bipartite graph and the unified graph matrix.

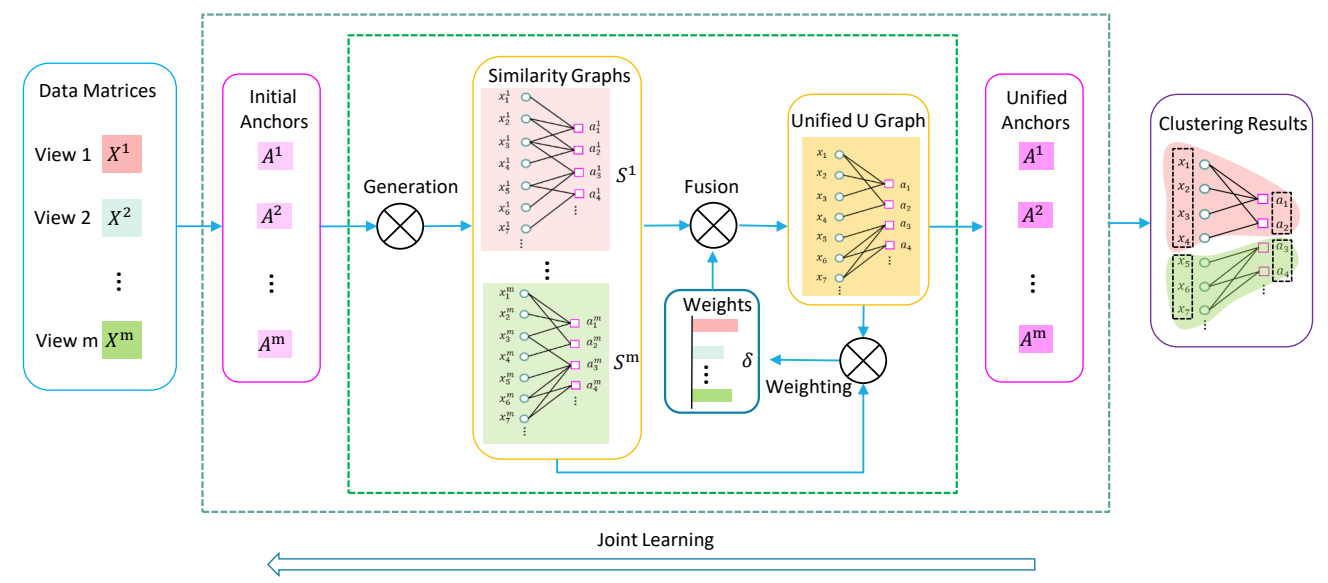

Figure 2. The framework of our proposed BIGMC method.

To address these limitations simultaneously, we propose a novel multi-view clustering approach, denoted by BIpartite Graph-based Multi-view Clustering (BIGMC) [41]. The overall framework of BIGMC is shown in Figure 2. To be specific, from the input of multi-view data matrices, we create $t$ initial uniform anchor points for different views denoted as $A$. Then the graph of each view is generated based on the similarity between data points and the anchor points, which is referred to as "data-to-anchor" similarity graph and denoted as S. Afterward, 
all $S$ s from multiple views are employed to learn a unified graph matrix $U$ in the fusion procedure. In the meantime, a weight for each view $(\delta)$ is added adaptively based on $S$ s and $U$ indicating its importance. A low-rank constraint is imposed on the Laplacian matrix of a unified bipartite graph associated with $U$, which aims to constrain that the bipartite graph has a $c$ number of connected components corresponding to the required number of clusters. Next, the obtained unified matrix $U$ would go back to improve the $S$ s and $\delta$ of each view until convergence. According to the converged unified graph matrix $U$, we can get the unified anchor points $A$ for each view. If they are different from the initial anchor points, we would improve all $A$ s to in turn update the $S$ s, the unified graph matrix $U$, and the weight $\delta$ until they are identical. The final clusters are formed directly based on $U$.

Hence, the main contributions can be summarized as follows.

- We propose a novel bipartite graph based multi-view clustering (BIGMC) approach. BIGMC can learn and make good use of the consensus information represented by a small number of uniform anchor points, which alleviates the influence of biases contained in multiple views.

- BIGMC jointly learns the similarity bipartite graph for each view, the unified bipartite graph, and the consensus anchors in a mutually reinforcing way. It can also determine the weight for each bipartite graph automatically without introducing an additive hyper-parameter. The final clusters are generated directly based on the unified bipartite graph when the anchors are identical in different views.

- BIGMC employs an efficient alternating iterative optimization strategy to solve the variable optimization problem step by step, where each sub-problem has an optimal solution. 
- Experimental results on both synthetic and real-world data sets demonstrate the effectiveness of the proposed BIGMC and the superiority over the stateof-the-art baselines.

The rest of this chapter is organized as follows. Section 2.2 gives a brief introduction of related multi-view clustering methods. Section 2.3 presents the proposed bipartite graph based multi-view clustering approach. The optimization strategy of this problem is given in Section 2.4. Extensive experiments are shown in Section 2.5. At last, Section 2.6 concludes this chapter.

\subsection{Related Work}

The existing graph-based multi-view clustering methods are related to the above-mentioned multi-view spectral clustering methods $[18,19]$. The difference is that the former method forms clusters on the unified graph of multiple views not on the embedding representation [42]. For most graph-based multi-view clustering methods, they still cannot simultaneously address the limitations mentioned in the introduction. For example, the authors in [19] utilize a two-state learning strategy, where they first construct the initial graph of each view and then optimize as well as integrate them into a global graph. Both [43] and [18] propose to learn a common graph directly without considering the discriminative information contained in different views. A graph-based multi-view clustering method [38] is proposed to jointly learn multiple view graphs and a fusion graph. It does not take the consensus information into account and also has high computational complexity. To this end, two multi-view spectral clustering methods via bipartite graph are presented $[39,15]$. While [39] keeps the selected salient points fixed and thus is sensitive to the initialization. Both [39] and [15] construct the Laplacian matrix for each view and keep them fixed during fusion. Additionally, K-means is required to obtain the final clusters. Our proposed BIGMC can alleviate all 
these limitations. In the experiment section, some representative methods will be compared to our method.

\subsection{Proposed Method}

Before presenting our proposed BIGMC method, we first introduce some notations. Throughout the paper, for a matrix $X \in \mathbb{R}^{d \times n}$, let $\mathrm{x}_{j}$ be the $j$-th column vector, $x_{i j}$ be the $(i, j)$-th entry, $\operatorname{Tr}(X)$ be the trace, and $\|X\|_{F}$ be the Frobenius norm respectively. For a vector $\mathrm{x} \in \mathbb{R}^{d \times 1}$, we denote $x_{j}$ as $j$-th entry, $\mathrm{x}^{T}$ as the transpose, and $\|\mathrm{x}\|_{p}=\left(\sum_{i=1}^{d}\left|x_{i}\right|^{p}\right)^{1 / p}$ as $l_{p^{-n o r m}}$. The identity matrix can be denoted by $\mathbf{I}$, and a vector with all entries of one can be denoted by $\mathbf{1}$.

The bipartite graph can be learned based on the similarities between data points and their corresponding neighbor anchor points [39]. For a multi-view data set with $m$ views, we denote $X^{1}, \ldots, X^{m}$ as the data matrices and $X^{v}=$ $\left[\mathrm{x}_{1}^{v}, \ldots, \mathrm{x}_{n}^{v}\right] \in \mathbb{R}^{d_{v} \times n}$ as the $v$-th view data with $d_{v}$ dimensions as well as $n$ data

points. For $X^{v}$, let $\mathrm{x}_{j}^{v}$ be the $j$-th column vector and $x_{i j}^{v}$ be the $(i, j)$-th entry. Let $A^{1}, \ldots, A^{m}$ be the uniform anchor matrices and $A^{v}=\left[\mathrm{a}_{1}^{v}, \ldots, \mathrm{a}_{t}^{v}\right] \in \mathbb{R}^{d_{v} \times t}$ as the anchor matrix of $X^{v}$ with $d_{v}$ dimensions as well as $t$ anchor points. $c$ is the required number of clusters. It is noteworthy that all view data have $t$ consensus anchor points, where each anchor point is the centroid of the corresponding subcluster. When $t=c$, each cluster only has one anchor point. When $c<t<n$, each cluster can be represented by several sub-clusters and thus has several anchor points. The specific number of the anchor points for each cluster can be learned by our proposed BIGMC method.

\subsubsection{View Graph Learning}

The similarity matrices between data and anchors can be denoted as $S^{1}, \ldots, S^{m}$, where $S^{v} \in \mathbb{R}^{n \times t}$. For the $i$-th data point $\mathrm{x}_{i}^{v}$ of $X^{v}$, we can connect the 
$j$-th anchor $a_{j}^{v}$ to it as a neighboring anchor with the probability $s_{i j}^{v}$. In general, closer $\mathrm{x}_{i}^{v}$ and $\mathrm{a}_{j}^{v}$ are likely to have larger connection probability $s_{i j}^{v}$ [38]. Thus $s_{i j}^{v}$ is inversely proportional to the distance between them, e.g. $\left\|x_{i}^{v}-a_{j}^{v}\right\|$. Therefore, when $\left\{A^{v}\right\}_{v=1}^{m}$ are fixed, the graphs for all views can be learned as follow:

$$
\min _{\left\{S^{v}\right\}_{v=1}^{m}} \sum_{v=1}^{m} \sum_{i=1}^{n} \sum_{j=1}^{t}\left\|\mathrm{x}_{i}^{v}-\mathrm{a}_{j}^{v}\right\|_{2}^{2} s_{i j}^{v}+\alpha \sum_{v=1}^{m}\left\|S^{v}\right\|_{F}^{2} \text { s.t. } \forall v, s_{i j}^{v} \geq 0, \mathbf{1}^{T} \mathrm{~s}_{i}^{v}=1 .
$$

where the second term is a regularization term, and the parameter $\alpha$ is employed to control the connection sparsity between data points and multiple anchors. If $\alpha=0$, there is a trivial solution for problem (1), i.e., $s_{i j}^{v}=1$ indicating that only its nearest anchor $\mathrm{a}_{j}^{v}$ can be connected to $\mathrm{x}_{i}^{v}$. This is called hard partition. If $\alpha$ is large enough, the connections from all $t$ anchors $\left\{\mathrm{a}_{j}^{v}\right\}_{j=1}^{t}$ to $\mathrm{x}_{i}^{v}$ can be built with the same probability $1 / t$. The value of $\alpha$ can be determined adaptively as shown in Section 2.4.1. The normalization $\mathbf{1}^{T} \mathrm{~S}_{i}^{v}=1$ can be considered as the sparse constraint on $S^{v}$.

Here, we learn the view graphs independently via constructing a similarity matrix for each view when fixing the anchor set. The reason is that each graph is only related to the other by the anchor set. Then, we produce a unified bipartite graph matrix and use it to update $\left\{A^{v}\right\}_{v=1}^{m}$ adaptively until convergence.

\subsubsection{Unified Graph Learning}

As mentioned above, our proposed BIGMC can jointly learn the graphs of all views, construct a unified bipartite graph, and automatically determine the importance of each view. To be specific, the unified bipartite graph can be obtained through a unified matrix $U \in \mathbb{R}^{n \times t}$ from $\left\{S^{v}\right\}_{v=1}^{m}$. Thus we have the following problem:

$$
\min _{U} \sum_{v=1}^{m}\left\|U-S^{v}\right\|_{F}^{2} \delta_{v} \text { s.t. } \forall i, u_{i j} \geq 0, \mathbf{1}^{T} \mathrm{u}_{i}=1
$$


where $\delta_{v}$ represents the weight of $v$-th view, $\mathrm{u}_{i} \in \mathbb{R}^{t \times 1}$ is a column vector of $U$, and $\mathrm{u}_{i j}$ is the $j$-th entry of $\mathrm{u}_{i}$. The values of the weights $\delta=\left\{\delta_{1}, \ldots, \delta_{m}\right\}$ can be determined automatically according to Theorem 1 [38] as follows:

Theorem 1. The weight $\delta_{v}$ can be determined by $\delta_{v}=\frac{1}{2 \sqrt{\left\|U-S^{v}\right\|_{F}^{2}}}$.

Proof. An auxiliary function is defined as follows:

$$
\min _{U} \sum_{v=1}^{m}\left\|U-S^{v}\right\|_{F} \text { s.t. } \forall i, u_{i j} \geq 0, \mathbf{1}^{T} \mathrm{u}_{i}=1
$$

The Lagrange function of Problem (3) can be written as:

$$
\sum_{v=1}^{m}\left\|U-S^{v}\right\|_{F}+\Theta(\Lambda, U)
$$

where $\Theta(\Lambda, U)$ is the formalized term derived from the constraints in problem (3), and $\Lambda$ is the Lagrange multiplier. Then we take the derivative of Problem (4) with respect to $U$ and set it to zero.

$$
\sum_{v=1}^{m}\left(\widehat{\delta_{v}}\right) \frac{\partial\left\|U-S^{v}\right\|_{F}^{2}}{\partial U}+\frac{\partial \Theta(\Lambda, U)}{\partial U}=0
$$

where $\widehat{\delta_{v}}$ is given by

$$
\widehat{\delta_{v}}=\frac{1}{2 \sqrt{\left\|U-S^{v}\right\|_{F}^{2}}} .
$$

Then we obtain the Lagrange function of Eq. (2) as follows:

$$
\sum_{v=1}^{m}\left(\delta_{v}\right) \frac{\partial\left\|U-S^{v}\right\|_{F}^{2}}{\partial U}+\frac{\partial \Theta(\Lambda, U)}{\partial U}=0
$$

From Eq. (5) and (7), we can get the same solution to Eq. (3) and Eq. (2) if $\delta_{v}=\widehat{\delta_{v}}$. In this case, the solution to the weight $\delta_{v}$ is

$$
\delta_{v}=\frac{1}{2 \sqrt{\left\|U-S^{v}\right\|_{F}^{2}}} .
$$

Eq. (5) cannot be solved directly since $\delta_{v}$ depends on the target variable $U$ when $S^{v}$ is given. However, if $\delta_{v}$ is set stationary, Eq. (5) can be regarded as the solution 
to Eq. (2). In this case, the calculated $U$ from Eq. (5) (it is in fact Eq. (22) shown below) will be further employed to update $\delta_{v}$ via Eq. (8). This strategy inspires us to solve the Problem (3) through an iterative way. Moreover, if the iterative optimization strategy converges (shown in Section 2.4), the converged values of $U$ and $S^{v}$ are optimal. Similarly, the weight $\delta_{v}$ is correspondingly tuned to an optimal value by Eq. (8). Hence, Problem (2) can be transformed into problem (3) when the weights $\delta$ are determined by Eq. (8), where the values of $U$ and $S^{v}$ are obtained in the last iteration.

Problem (1) and Problem (2) can be combined to learn $\left\{S^{v}\right\}_{v=1}^{m}$ and $U$ jointly as follows:

$$
\begin{aligned}
& \min _{\left\{S^{v}\right\}_{v=1}^{m}, U} \sum_{v=1}^{m} \sum_{i=1}^{n} \sum_{j=1}^{t}\left\|\mathrm{x}_{i}^{v}-\mathrm{a}_{j}^{v}\right\|_{2}^{2} s_{i j}^{v}+\alpha \sum_{v=1}^{m}\left\|S^{v}\right\|_{F}^{2}+\sum_{v=1}^{m}\left\|U-S^{v}\right\|_{F}^{2} \delta_{v} \\
& \text { s.t. } \forall v, i, s_{i j}^{v} \geq 0, \mathbf{1}^{T} \mathrm{~s}_{i}^{v}=1, u_{i j} \geq 0, \mathbf{1}^{T} \mathrm{u}_{i}=1 .
\end{aligned}
$$

We notice that the matrices $\left\{S^{v}\right\}_{v=1}^{m}$ and $U$ can be learned jointly in a problem when $\left\{A^{v}\right\}_{v=1}^{m}$ are fixed. In the next subsection, we can adaptively find the consensus anchor points of all views.

\subsubsection{Consensus Anchor Learning}

When the unified matrix $U$ is updated, we can explore the consensus anchors and reposition them in all views. For $j$-th subcluster of $v$-th view data, its anchor $\mathrm{a}_{j}^{v}$ can be obtained based on the mean of all data points connected to it by

$$
\mathrm{a}_{j}^{v}=\frac{\sum_{i=1}^{n} u_{i j} \mathrm{x}_{i}^{v}}{\sum_{i=1}^{n} u_{i j}}
$$

where $\mathrm{a}_{j}^{v} \in \mathbb{R}^{d_{v} \times 1}$ and $j=1, \ldots, t$. Then the anchor matrices $\left\{A^{v}\right\}_{v=1}^{m}$ can be updated. At last, we combine Eq. (10) with problem (9) and learn the matrices $\left\{S^{v}\right\}_{v=1}^{m}, U$, and $\left\{A^{v}\right\}_{v=1}^{m}$ jointly such that they can assist each other in the iteration 
process.

$$
\begin{aligned}
& \min _{\left\{S^{v}\right\}_{v=1}^{m}, U,\left\{A^{v}\right\}_{v=1}^{m}} \sum_{v=1}^{m} \sum_{i=1}^{n} \sum_{j=1}^{t}\left\|\mathrm{x}_{i}^{v}-\mathrm{a}_{j}^{v}\right\|_{2}^{2} s_{i j}^{v}+\alpha \sum_{v=1}^{m}\left\|S^{v}\right\|_{F}^{2}+\sum_{v=1}^{m}\left\|U-S^{v}\right\|_{F}^{2} \delta_{v} \\
& \text { s.t. } \forall v, i, s_{i j}^{v} \geq 0, \mathbf{1}^{T} \mathrm{~s}_{i}^{v}=1, u_{i j} \geq 0, \mathbf{1}^{T} \mathrm{u}_{i}=1 .
\end{aligned}
$$

\subsubsection{Optimal Bipartite Graph Learning}

As mentioned above, the edge weights of the bipartite graph can be represented by $U \in \mathbb{R}^{n \times t}$, where each element, $u_{i j}$, is a weight of the edge that connects $\mathrm{x}_{i}$ and the corresponding $\mathrm{a}_{j}$ of all views [44]. In this case, the weighted adjacency matrix, $Z \in \mathbb{R}^{(n+t) \times(n+t)}$, and the degree matrix, $D_{U}$, can have the following block structures:

$$
Z=\left[\begin{array}{cc}
0 & U \\
U^{T} & 0
\end{array}\right], D_{U}=\left[\begin{array}{cc}
D_{1} & 0 \\
0 & D_{2}
\end{array}\right]
$$

where $D_{1} \in \mathbb{R}^{n \times n} ; i$-th vector of $D_{1}$ is $\mathrm{d}_{i}^{1}=\sum_{j=1}^{t} u_{i j} ; D_{2} \in \mathbb{R}^{t \times t} ; j$-th vector of $D_{2}$ is $\mathrm{d}_{j}^{2}=\sum_{i=1}^{n} u_{i j}$. Hence, the normalized Laplacian matrix is given by

$$
L_{U}=\mathbf{I}-\left(D_{U}\right)^{-1 / 2} Z\left(D_{U}\right)^{-1 / 2}
$$

The neighbor anchor assignment is optimal for each data point in all views when there exist exactly $c$ connected components in the bipartite graph. It can be achieved by imposing a rank constraint on $L_{U}$ of the bipartite graph $Z$ associated with $U$. As pointed out by [45], first, the eigenvalues of $L_{U}$ are in a normalized form that enables the spectra to relate better to graph invariants for general graphs to some extent than a standard form. Second, there is an important property of $D_{U}$ if $U$ is non-negative:

Theorem 2. The multiplicity $c$ of the eigenvalue 0 of the normalized Laplacian matrix $L_{U}$ equals the number of connected components in the bipartite graph associated with $U$. 
The proof of Theorem 2 has been shown in [45]. The Theorem 1 says that the $n$ data points and $t$ anchors can be partitioned into $c$ clusters based on $Z$ related to $U$ if $\operatorname{rank}\left(L_{U}\right)=(n+t)-c$. Hence, the final subclusters and clusters can be generated without need to perform an additional clustering method. The optimal bipartite graph can be learned by solving the following problem:

$$
\begin{aligned}
& \min _{\left\{S^{v}\right\}_{v=1}^{m}, U,\left\{A^{v}\right\}_{v=1}^{m}} \sum_{v=1}^{m} \sum_{i=1}^{n} \sum_{j=1}^{t}\left\|\mathrm{x}_{i}^{v}-\mathrm{a}_{j}^{v}\right\|_{2}^{2} s_{i j}^{v}+\alpha \sum_{v=1}^{m}\left\|S^{v}\right\|_{F}^{2}+\sum_{v=1}^{m}\left\|U-S^{v}\right\|_{F}^{2} \delta_{v} \\
& \text { s.t. } \forall v, i, s_{i j}^{v} \geq 0, \mathbf{1}^{T} \mathrm{~s}_{i}^{v}=1, u_{i j} \geq 0, \mathbf{1}^{T} \mathrm{u}_{i}=1, \operatorname{rank}\left(L_{U}\right)=(n+t)-c .
\end{aligned}
$$

It can be noticed that the constraint $\operatorname{rank}\left(L_{U}\right)=(n+t)-c$ is nonlinear and hard to solve. To relax this constraint, we introduce $c$-smallest eigenvalues of $L_{U}$, denoted by $\left\{\eta_{q}\left(L_{U}\right)\right\}_{q=1}^{c}$, in which $\eta_{q}\left(L_{U}\right) \geq 0$ since $L_{U}$ is positive semi-definite. Thus let $\sum_{q=1}^{c} \eta_{q}\left(L_{U}\right)=0$ so that the rank constraint can be achieved. From Ky Fan's Theorem [46], this problem can be turned into

$$
\sum_{q=1}^{c} \eta_{q}\left(L_{U}\right)=\min _{F \in \mathbb{R}^{(n+t) \times c, F^{T}} F=\mathbf{I}} \operatorname{Tr}\left(F^{T} L_{U} F\right) .
$$

Therefore, we can obtain the objective function by plugging problem (14) into problem (13).

$$
\begin{aligned}
& \min _{\left\{S^{v}\right\}_{v=1}^{m}, U,\left\{A^{v}\right\}_{v=1}^{m}, F} \sum_{v=1}^{m} \sum_{i=1}^{n} \sum_{j=1}^{t}\left\|\mathrm{x}_{i}^{v}-\mathrm{a}_{j}^{v}\right\|_{2}^{2} s_{i j}^{v}+\alpha \sum_{v=1}^{m}\left\|S^{v}\right\|_{F}^{2} \\
& +\sum_{v=1}^{m}\left\|U-S^{v}\right\|_{F}^{2} \delta_{v}+\beta \operatorname{Tr}\left(F^{T} L_{U} F\right) \\
& \text { s.t. } \forall v, i, s_{i j}^{v} \geq 0, \mathbf{1}^{T} \mathrm{~s}_{i}^{v}=1, u_{i j} \geq 0, \mathbf{1}^{T} \mathrm{u}_{i}=1, F^{T} F=\mathbf{I} .
\end{aligned}
$$

When the parameter $\beta$ is large enough, the optimal $U$ obtained by solving problem (15) can make $\sum_{q=1}^{c} \eta_{q}\left(L_{U}\right)=0$ achieved. Note that we can use $\beta$ to control the number of connected components in the bipartite graph, denoted by $\gamma$. $\beta$ will be increased when $\gamma<c$ and decreased when $\gamma>c$ in each iteration. Hence, the resulting bipartite graph matrix $Z$ has exact $c$ connected components, and groups 
$n$ data points as well as $t$ anchors into $c$ clusters. We can solve the problem (15) by an alternating optimization strategy.

\subsection{Optimization Strategy}

It has been a challenging issue that each variable in the Problem (15) can have an optimized solution since they are coupled together. An alternating iterative strategy [39] can effectively transform a constrained optimization problem into a series of unconstrained sub-problems by plugging some penalty terms into the objective function. In this chapter, we have variables $\{S\}_{v=1}^{m},\left\{\delta_{v}\right\}_{v=1}^{m}, U, F$, and $\left\{A_{v}\right\}_{v=1}^{m}$ to be optimized. The strategy is that one of them is updated when the others are fixed. Specifically, the updated rules are presented in the subsections.

\subsubsection{Fix $\left\{\delta_{v}\right\}_{v=1}^{m}, U, F$, and $\left\{A_{v}\right\}_{v=1}^{m}$, Update $\{S\}_{v=1}^{m}$}

When we fix $\left\{\delta_{v}\right\}_{v=1}^{m}, U, F$, and $\left\{A_{v}\right\}_{v=1}^{m}$ of the problem (15), which makes the last term a constant. In this case, the problem becomes:

$$
\begin{aligned}
& \min _{\left\{S^{v}\right\}_{v=1}^{m}} \sum_{v=1}^{m} \sum_{i=1}^{n} \sum_{j=1}^{t}\left\|\mathrm{x}_{i}^{v}-\mathrm{a}_{j}^{v}\right\|_{2}^{2} s_{i j}^{v}+\alpha \sum_{v=1}^{m}\left\|S^{v}\right\|_{F}^{2}+\sum_{v=1}^{m}\left\|U-S^{v}\right\|_{F}^{2} \delta_{v} \\
& \text { s.t. } \forall v, i, s_{i j}^{v} \geq 0, \mathbf{1}^{T} \mathrm{~s}_{i}^{v}=1 .
\end{aligned}
$$

It is easy to be noticed that the updates of $\{S\}_{v=1}^{m}$ are independent for all views and not coupled together. Thus, $S^{v}$ can be updated individually by the following problem:

$$
\begin{aligned}
& \min _{S^{v}} \sum_{i=1}^{n} \sum_{j=1}^{t}\left\|\mathrm{x}_{i}^{v}-\mathrm{a}_{j}^{v}\right\|_{2}^{2} s_{i j}^{v}+\alpha\left\|S^{v}\right\|_{F}^{2}+\left\|U-S^{v}\right\|_{F}^{2} \delta_{v} \\
& \text { s.t. } \forall i, s_{i j}^{v} \geq 0, \mathbf{1}^{T} \mathrm{~s}_{i}^{v}=1 .
\end{aligned}
$$

Besides, we can also find that updating $\mathrm{s}_{i}^{v}$ for each vector is independent and 
get $\mathrm{s}_{i}^{v}$ by optimizing the following function:

$$
\begin{aligned}
& \min _{\mathrm{s}_{i}^{v}} \sum_{j=1}^{t}\left\|\mathrm{x}_{i}^{v}-\mathrm{a}_{j}^{v}\right\|_{2}^{2} s_{i j}^{v}+\alpha\left\|\mathrm{s}_{i}^{v}\right\|_{2}^{2}+\left\|\mathrm{u}_{i}-\mathrm{s}_{i}^{v}\right\|_{2}^{2} \delta_{v} \\
& \text { s.t. } \forall i, \quad s_{i j}^{v} \geq 0, \mathbf{1}^{T} \mathrm{~s}_{i}^{v}=1 .
\end{aligned}
$$

For the convenience of calculation, let $\theta_{i}$ as a vector with $j$-th element $\theta_{i j}=$ $\left\|\mathrm{x}_{i}^{v}-\mathrm{a}_{j}^{v}\right\|$. Then the problem (18) can be rewritten as

$$
\begin{aligned}
& \min _{\mathrm{s}_{i}^{v}} \frac{1}{2}\left\|\mathrm{~s}_{i}^{v}+\frac{\theta_{i}}{2 \alpha}\right\|_{2}^{2}+\frac{1}{2 \alpha}\left\|\mathrm{u}_{i}-\mathrm{s}_{i}^{v}\right\|_{2}^{2} \delta_{v} \\
& \text { s.t. } \forall i, s_{i j}^{v} \geq 0, \mathbf{1}^{T} \mathrm{~s}_{i}^{v}=1 .
\end{aligned}
$$

The problem (19) can be tackled with a closed-form if we constrain $\mathrm{s}_{i}^{v}$ having $k$ nonzero elements. That is to say, only $k$-nearest anchors for each data point $\mathrm{x}_{i}^{v}$ are taken into account instead of $k$-nearest data points. This assignment of multiple neighboring anchors contributes to preserving both the invariant and discriminative local structures since each object in different views has not only invariances but also discrepancies. From [38], we have

$$
\alpha=\frac{1}{2}\left(k \theta_{i, k+1}-\sum_{a=1}^{k} \theta_{i a}-2 k \delta_{v} u_{i, k+1}-2 \delta_{v}\right)
$$

and the final optimized solution of $\mathrm{s}_{i j}^{v}$

$$
\mathrm{s}_{i j}^{v}= \begin{cases}\frac{\theta_{i, k+1}-\theta_{i j}+2 \delta_{v}\left(u_{i j}-u_{i, k+1}\right)}{k \theta_{i, k+1}-\sum_{a=1}^{k} \theta_{i a}-2 k \delta_{v} u_{i, k+1}+2 \sum_{a=1}^{k} \delta_{v} u_{i a}} & j \leq k \\ 0 & j>k\end{cases}
$$

\subsubsection{Fix $\{S\}_{v=1}^{m}, U, F$, and $\left\{A_{v}\right\}_{v=1}^{m}$, Update $\left\{\delta_{v}\right\}_{v=1}^{m}$}

When we fix $\{S\}_{v=1}^{m}, U, F$, and $\left\{A_{v}\right\}_{v=1}^{m}$, solving problem (15) to update $\left\{\delta_{v}\right\}_{v=1}^{m}$ can be turned into solving problem (2). As mentioned above, the final solution of each $\delta_{v}$ in $\left\{\delta_{v}\right\}_{v=1}^{m}$ can be obtained according to Eq. (8). 


\subsubsection{Fix $\{S\}_{v=1}^{m},\left\{\delta_{v}\right\}_{v=1}^{m}, F$, and $\left\{A_{v}\right\}_{v=1}^{m}$, Update $U$}

When we fix $\{S\}_{v=1}^{m},\left\{\delta_{v}\right\}_{v=1}^{m}, F$, and $\left\{A_{v}\right\}_{v=1}^{m}$, the problem (15) can be transformed into

$$
\begin{aligned}
& \min _{U} \sum_{v=1}^{m}\left\|U-S^{v}\right\|_{F}^{2} \delta_{v}+\beta \operatorname{Tr}\left(F^{T} L_{U} F\right) \\
& \text { s.t. } \forall i, u_{i j} \geq 0, \mathbf{1}^{T} \mathrm{u}_{i}=1 .
\end{aligned}
$$

where all $L_{U}, D_{U}$, and $Z$ depend on $U$. Specifically, the last term reveals the mutual relations as follows:

$$
\operatorname{Tr}\left(F^{T} L_{U} F\right)=\frac{1}{2} \sum_{i=1}^{n+t} \sum_{j=1}^{n+t} z_{i j}\left\|\frac{\mathrm{f}_{i}}{\mathrm{~d}_{i}^{1}}-\frac{\mathrm{f}_{j}}{\mathrm{~d}_{j}^{2}}\right\|_{2}^{2}=\sum_{i=1}^{n} \sum_{j=1}^{t} u_{i j} \mu_{i j}
$$

where $\mu_{i j}=\left\|\frac{\mathrm{f}_{i}}{\mathrm{~d}_{i}^{1}}-\frac{\mathrm{f}_{j}}{\mathrm{~d}_{j}^{2}}\right\|_{2}^{2}$. Then, the problem (22) can be rewritten as:

$$
\begin{aligned}
& \min _{U} \sum_{v=1}^{m}\left\|U-S^{v}\right\|_{F}^{2} \delta_{v}+\beta \sum_{i=1}^{n} \sum_{j=1}^{t} u_{i j} \mu_{i j} \\
& \text { s.t. } \forall i, u_{i j} \geq 0, \mathbf{1}^{T} \mathrm{u}_{i}=1 .
\end{aligned}
$$

Similarly, updating $\mathrm{u}_{i}$ for each row in $U$ is independent. We can have

$$
\begin{aligned}
& \min _{\mathrm{u}_{i}} \sum_{v=1}^{m}\left\|\mathrm{u}_{i}-\mathrm{s}_{i}^{v}\right\|_{2}^{2} \delta_{v}+\beta \mu_{i}^{T} \mathrm{u}_{i} \\
& \text { s.t. } \forall i, u_{i j} \geq 0, \mathbf{1}^{T} \mathrm{u}_{i}=1 .
\end{aligned}
$$

We denote $\phi$ and $\varphi$ as the Lagrange multipliers for the two constraints. Thus we can have the Lagrange function of problem (25):

$$
\mathcal{L}\left(\mathrm{u}_{i}, \phi, \varphi\right)=\sum_{v=1}^{m}\left\|\mathrm{u}_{i}-\mathrm{s}_{i}^{v}\right\|_{2}^{2} \delta_{v}+\beta \mu_{i}^{T} \mathrm{u}_{i}-\phi\left(\mathbf{1}^{T} \mathrm{u}_{i}-1\right)-\varphi^{T} \mathrm{u}_{i} .
$$

Then, we take the derivative of $\mathcal{L}$ with respect to $\mathrm{u}_{i}$, set it to zero, and obtain the following equation:

$$
2 \mathrm{u}_{i} \sum_{v=1}^{m} \delta_{v}-2 \sum_{v=1}^{m} \mathrm{~s}_{i}^{v} \delta_{v}+\beta \mu_{i}-\phi \mathbf{1}-\varphi=\mathbf{0}
$$


From [47], only the optimal $u_{i}^{*}, \phi^{*}$, and $\varphi^{*}$ can satisfy the Eq. (27). Additionally, $\beta$ can be adaptively determined and thus be treated as a known parameter. Let $a=2 \sum_{v=1}^{m} \delta_{v}$ and $\mathrm{p}_{i}=2 \sum_{v=1}^{m} \mathrm{~s}_{i}^{v} \delta_{v}-\beta \mu_{i}$ for the constants. On the basis of the Karush-Kuhn-Tucker (KKT) conditions, we can have

$$
\mathrm{u}_{i}^{*} a-\mathrm{p}_{i}-\phi^{*} \mathbf{1}-\varphi^{*}=\mathbf{0}
$$

where $\forall j, \mathrm{u}_{i j}^{*} \geq 0, \varphi_{j}^{*} \geq 0, \mathrm{u}_{i j}^{*} \varphi_{j}^{*}=0$. According to the constraint $\mathbf{1}^{T} \mathrm{u}_{i}=1$, i.e., $\mathbf{1}^{T} \mathrm{u}_{i}^{*}=1$ we can obtain

$$
\phi^{*}=\frac{a-\mathbf{1}^{T} \mathrm{p}_{i}-\mathbf{1}^{T} \varphi^{*}}{t}
$$

Plugging $\phi^{*}$ into Eq. (28), we can have

$$
\mathrm{u}_{i}^{*}=\frac{\mathrm{p}_{i}}{a}+\frac{\mathbf{1}}{t}-\frac{\mathbf{1}^{T} \mathrm{p}_{i} \mathbf{1}}{a t}-\frac{\mathbf{1}^{T} \varphi^{*} \mathbf{1}}{a t}+\frac{\varphi^{*}}{a}=\left(\mathrm{w}_{i}-\sigma^{*} \mathbf{1}\right)_{+}
$$

where $\mathrm{w}_{i}=\frac{\mathrm{p}_{i}}{a}+\frac{\mathbf{1}}{t}-\frac{\mathbf{1}^{T} \mathrm{p}_{i} \mathbf{1}}{a t}, \sigma^{*}=\frac{\mathbf{1}^{T} \varphi^{*}}{a t}, \frac{\varphi^{*}}{a} \geq 0,(\cdot)_{+}=\max (\cdot, 0)$, and $u_{i j}^{*}=$ $\left(w_{i j}-\sigma^{*}\right)_{+}$. We can also derive $\varphi_{j}^{*}=a\left(\sigma^{*}-w_{i j}+u_{i j}^{*}\right)=a\left(\sigma^{*}-w_{i j}\right)_{+}$. Thus $\sigma^{*}=\frac{\sum_{j=1}^{t}\left(\sigma^{*}-w_{i j}\right)_{+}}{t}$. The solution $\sigma^{*}$ can be obtained by finding the root of problem as

$$
f(\sigma)=\sigma-\frac{\sum_{j=1}^{t}\left(\sigma-w_{i j}\right)_{+}}{t}
$$

where $\sigma \geq 0$ and $f^{\prime}(\sigma) \geq 0$. It can be noted that $f(\sigma)$ is a linear and convex function. The Newton-Raphson method as a root-finding algorithm can generate a successively approximation to the root of a real-valued function. Hence, a sufficiently precise value of $\sigma$ (i.e., $\sigma^{*}$ ) is reached by iterating computing a better approximation, $\sigma_{\tau+1}$, to the root. Solving for $\sigma_{\tau+1}$ gives

$$
\sigma_{\tau+1}=\sigma_{\tau}-\frac{f(\sigma)}{f^{\prime}(\sigma)}
$$

Therefore, $\mathrm{u}_{i}^{*}$ can be obtained by Eq. (30) for each row of $U$. 


\subsubsection{Fix $\{S\}_{v=1}^{m},\left\{\delta_{v}\right\}_{v=1}^{m}, U$, and $\left\{A_{v}\right\}_{v=1}^{m}$, Update $F$}

When we fix $\{S\}_{v=1}^{m},\left\{\delta_{v}\right\}_{v=1}^{m}, U$, and $\left\{A_{v}\right\}_{v=1}^{m}, F$ can be updated by handling the following problem:

$$
\min _{F} \operatorname{Tr}\left(F^{T} L_{U} F\right) \text { s.t. } F^{T} F=\mathbf{I}
$$

We can rewrite $F$ as the block matrix

$$
F=\left[\begin{array}{l}
F_{1} \\
F_{2}
\end{array}\right]
$$

where $F_{1} \in \mathbb{R}^{n \times c}$ and $F_{2} \in \mathbb{R}^{t \times c}$. According to Eq. (12), the problem (33) can be rewritten as

$$
\max _{F^{T} F=\mathbf{I}} \operatorname{Tr}\left(F^{T}\left(D_{U}\right)^{-1 / 2} Z\left(D_{U}\right)^{-1 / 2} F\right) \Rightarrow \max _{F_{1}^{T} F_{1}+F_{2}^{T} F_{2}=\mathbf{I}} \operatorname{Tr}\left(F_{1}^{T}\left(D_{1}\right)^{-1 / 2} U\left(D_{2}\right)^{-1 / 2} F_{2}\right)
$$

The problem (34) can be solved by Lemma $1[48,44]$. In Lemma $1, B=$ $\left(D_{1}\right)^{-1 / 2} U\left(D_{2}\right)^{-1 / 2}$.

Lemma 1. Given $F_{1} \in \mathbb{R}^{n \times c}, B \in \mathbb{R}^{n \times t}$, and $F_{2} \in \mathbb{R}^{t \times c}$. The optimal solutions to the problem

$$
\max _{F_{1}^{T} F_{1}+F_{2}^{T} F_{2}=I} \operatorname{Tr}\left(F_{1}^{T} B F_{2}\right)
$$

are $F_{1}=\frac{\sqrt{2}}{2} B_{1}$ and $F_{2}=\frac{\sqrt{2}}{2} B_{2}$, in which $B_{1}$ are the leading c left singular vectors of $B$ and $B_{2}$ are the leading c right singular vectors of $B$.

The optimal $F$ is composed of the optimal $F_{1}$ and $F_{2}$.

\subsubsection{Fix $\{S\}_{v=1}^{m},\left\{\delta_{v}\right\}_{v=1}^{m}, U$, and $F$, Update $\left\{A_{v}\right\}_{v=1}^{m}$}

When we fix $\{S\}_{v=1}^{m},\left\{\delta_{v}\right\}_{v=1}^{m}, U$, and $F$, each $a_{j}^{v}$ can be updated by Eq. (10). The details are shown in Algorithm 1. 


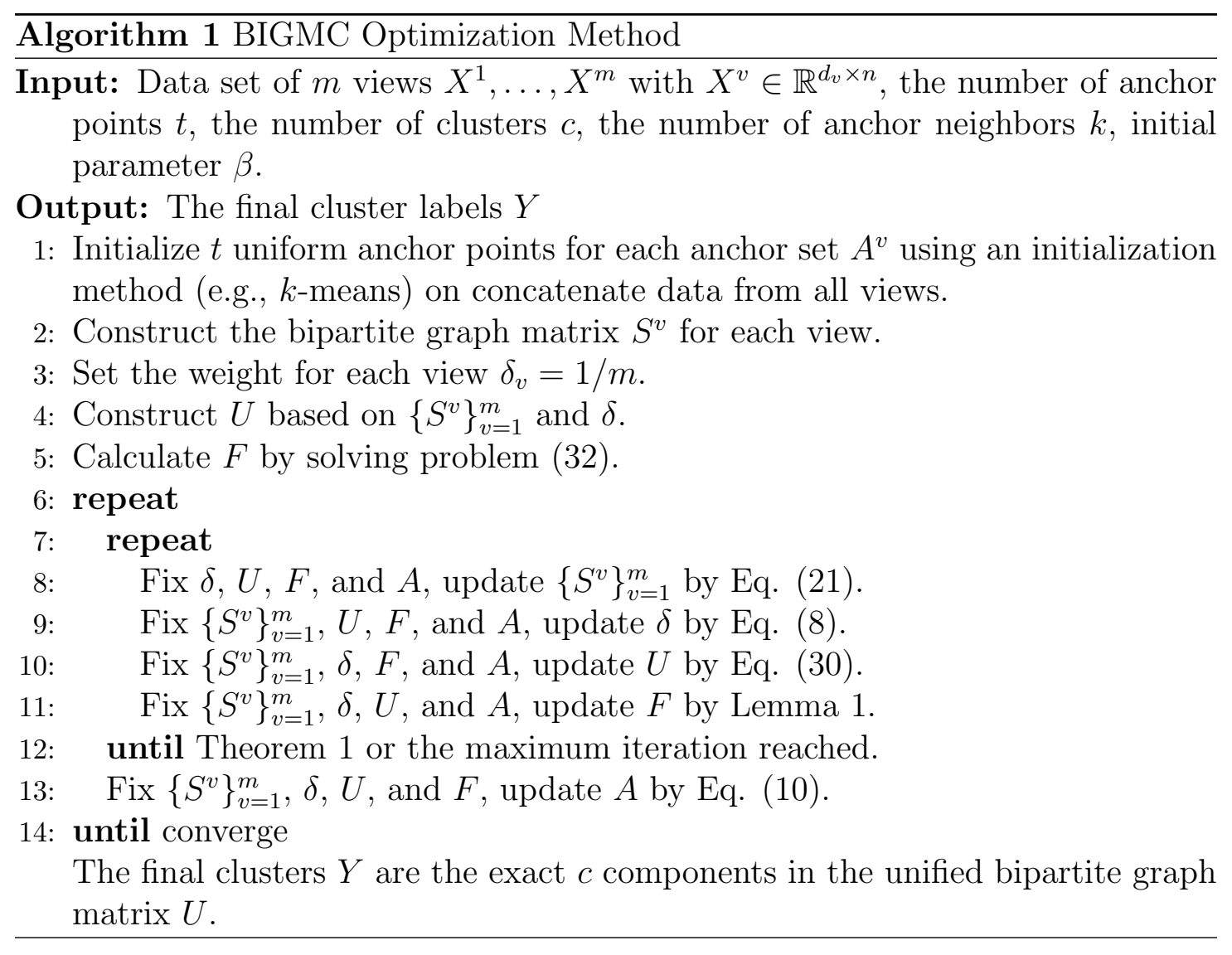

\subsection{Complexity and Convergence Analysis 2.5.1 Complexity Analysis}

From Algorithm 1, the computational complexity of our proposed BIGMC method consists mainly of six parts, which correspond the initialization and updates of our variables respectively. To be more specific, the update of $\left\{S^{v}\right\}_{v=1}^{m}$ takes $O(m n t)$, where $m$ is the number of views; $n$ is the number of data objects; $t$ is the number of anchor points and $c \leq t \ll n$; $c$ is the number of required number of clusters. The update of weights of all views $\delta$ has the computational complexity of $O(m n t)$. The update of the unified graph matrix $U$ is achieved by solving Eq. (28) taking $O(c n)$. The learning of $F$ takes $O(c n t)$. Hence, this sub-iteration procedure is $O\left((2 m t+c+c t) n \zeta_{1}\right)$, where $\zeta_{1}$ is the number of iterations. Updating the anchor points $A$ needs to cost $O(m n t d)$, where $d=\max \left(d^{1}, \ldots, d^{m}\right)$. Moreover, we initialize the anchors $\left\{A^{v}\right\}_{v=1}^{m}$ by taking $O(n d t)$ with Var-Part method. The 
initialization of $\left\{S^{v}\right\}_{v=1}^{m}$ takes $O(m n d t)$.

Overall, the computational complexity of BIGMC takes $O\left(\left((2 m t+c+c t) \zeta_{1}+\right.\right.$ $\left.m t d) n \zeta_{2}+n d t(m+1)\right)$, in which $\zeta_{2}$ is the number of iterations.

\subsubsection{Convergence Analysis}

The overall objective function Eq. (15) is not a joint convex optimization problem of variables. Acquiring a globally optimal solution is still an open problem. The problem (15) is solved using the optimization strategy proposed in Section 4. After alternating optimizing variables, the corresponding each sub-problem is convex and the optimal solution of it is given. Specifically, the convergences of all sub-problems can be shown as follows.

For the update of $\{S\}_{v=1}^{m}$, the objective function of problem (19) is a convex function. The reason for this conclusion is that its second order derivative with respect to $\mathrm{s}_{i}^{v}$ is equal to 1 . Therefore, it is monotonic decreasing using the optimization strategy.

For the update of weights $\delta$, the objective function of problem (2) is a linear convex problem. A closed-form solution of $\delta$ is given in Eq. (8).

For the update of $U$, we can denote $\widehat{U}$ as the updated $U$ in the augmented Lagrangian iteration process and $\Gamma(U)=\beta \operatorname{Tr}\left(F^{T} L_{U} F\right)$. Thus the following inequality can be derived from problem (23) and (8) with the decrease of function error

$$
\sum_{v=1}^{m} \frac{\left\|\widehat{U}-S^{v}\right\|_{F}^{2}}{2\left\|\widehat{U}-S^{v}\right\|_{F}}+\Gamma(\widehat{U}) \leq \sum_{v=1}^{m} \frac{\left\|U-S^{v}\right\|_{F}^{2}}{2\left\|U-S^{v}\right\|_{F}}+\Gamma(U) .
$$

According to a lemma from [49], the convergence of problem (22) can be obtained.

Lemma 2. For any non-zero matrix $A^{\prime} \in \mathbb{R}^{n \times t}$ and $B^{\prime} \in \mathbb{R}^{n \times t}$, the following 
inequality holds:

$$
\left\|A^{\prime}\right\|_{F}-\frac{\left\|A^{\prime}\right\|_{F}^{2}}{2\left\|B^{\prime}\right\|_{F}^{2}} \leq\left\|B^{\prime}\right\|_{F}-\frac{\left\|B^{\prime}\right\|_{F}^{2}}{2\left\|B^{\prime}\right\|_{F}^{2}}
$$

Let $A^{\prime}=\sum_{v=1}^{m}\left(\widehat{U}-S^{v}\right)$ and $B^{\prime}=\sum_{v=1}^{m}\left(U-S^{v}\right)$, and we can have

$$
\begin{aligned}
& \left\|\sum_{v=1}^{m}\left(\widehat{U}-S^{v}\right)\right\|_{F}-\frac{\left\|\sum_{v=1}^{m}\left(\widehat{U}-S^{v}\right)\right\|_{F}^{2}}{2\left\|\sum_{v=1}^{m}\left(U-S^{v}\right)\right\|_{F}^{2}} \leq \\
& \left\|\sum_{v=1}^{m}\left(U-S^{v}\right)\right\|_{F}-\frac{\left\|\sum_{v=1}^{m}\left(U-S^{v}\right)\right\|_{F}^{2}}{2\left\|\sum_{v=1}^{m}\left(U-S^{v}\right)\right\|_{F}^{2}}
\end{aligned}
$$

Therefore,

$$
\begin{aligned}
& \sum_{v=1}^{m}\left\|\widehat{U}-S^{v}\right\|_{F}-\sum_{v=1}^{m} \frac{\left\|\widehat{U}-S^{v}\right\|_{F}^{2}}{2\left\|U-S^{v}\right\|_{F}^{2}} \leq \\
& \sum_{v=1}^{m}\left\|U-S^{v}\right\|_{F}-\sum_{v=1}^{m} \frac{\left\|U-S^{v}\right\|_{F}^{2}}{2\left\|U-S^{v}\right\|_{F}^{2}}
\end{aligned}
$$

We sum Eq. (35) and (38) over both sides and get

$$
\sum_{v=1}^{m}\left\|\widehat{U}-S^{v}\right\|_{F}+\Gamma(\widehat{U}) \leq \sum_{v=1}^{m}\left\|U-S^{v}\right\|_{F}+\Gamma(U)
$$

As a result, the convergence of (22) is proved.

For the update of $F$, the objective function of problem (33) $F$ is updated by Lemma 1 through SVD of $B$.

For the update of $\{A\}_{v=1}^{m}$, the problem converges when the connections between data points and anchor points no longer change.

\subsection{Experiments}

The experiments are conducted on Matlab development environment to compare with the baseline methods. In this section, we investigate the performance of our proposed BIGMC method on both synthetic and real-world data sets. Thus, we present two groups of experiments. The first group is to show the effectiveness of BIGMC by observing the visual illustration of its capability on the synthetic data sets. The learned connections within each view will be shown to prove the 
capability of similarity learning, where a large line weight indicates a strong connection between the data point and its neighbor anchor point. The second group can be divided into five sub-groups. The first sub-group is to determine an efficient initialization method for BIGMC to initialize the anchor points such that the sensitivity to the initialization can be alleviated. The second sub-group contains the clustering results on real-world datasets to demonstrate the superiority of BIGMC compared with the baselines. Moreover, the learning results of anchors by BIGMC on 3 real-world datasets are presented to show the adaptive ability to learn anchors. The third sub-group is to further evaluate BIGMC by generating 4 variants of BIGMC. The fourth sub-group is to show the convergence results of BIGMC. The fifth sub-group contains the results of running time by BIGMC and baselines. In this chapter, we assume all views are complete.

\subsubsection{Experiments on Synthetic Datasets}

Data sets. We follow $[19,38]$ to conduct experiments on two synthetic datasets to evaluate the performance of our proposed BIGMC. The first one contains two views, which are shown in Figure 3 (a) and (d), called "Two-Moon data set". Each view has two clusters, i.e., one moon pattern with red dots and the other moon pattern with blue dots. Each cluster has 100 data points and adds 0.12 percentage of random Gaussian noises. The second also involves two views, which are shown in Figure $4(\mathrm{a})$ and $(\mathrm{d})$, called "Three-Circle data set". Each view has three clusters respectively represented by 30 red dots, 90 blue dots, and 180 black dots in a circle pattern. The same percentage of random Gaussian noises are added.

Results. For the Two-Moon data set, we set the number of anchor neighbors $k=3$ and the number of anchor points $t=20$. Figure $3(\mathrm{~b})$ and (e) show the learned graphs for the two views, in which the pink squares are the learned anchor 


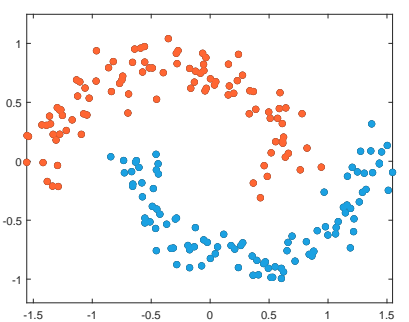

(a)

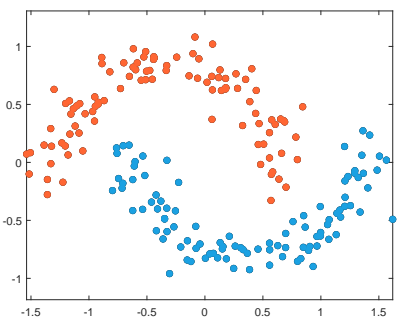

(d)

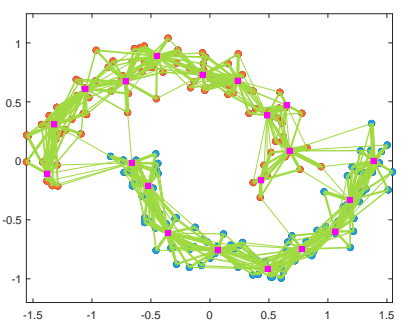

(b)

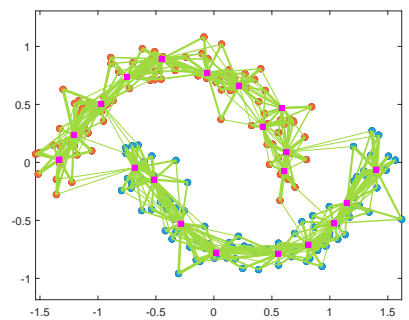

(e)

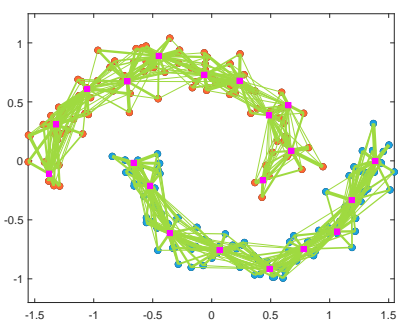

(c)

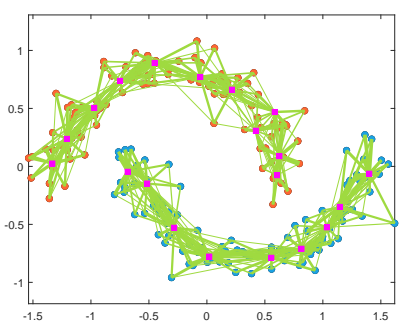

(f)

Figure 3. Clustering results on Two-Moon data set. The upper row includes the original first view data, the learned graph with the learned $S^{1}$, and the learned graph with the learned $U$. The lower row contains the original second view data, the learned graph with the learned $S^{2}$, and the learned graph with the learned $U$. The red dots are cluster 1 , and the blue dots are cluster 2 . The pink squares are the learned anchor points, and the green lines are the learned connections between data points and anchor points.

points, and lines are generated from the learned $S^{1}$ and $S^{2}$, respectively. The line weight indicates how much similar each data point and its neighbor anchor point. It can be seen that the two clusters are weakly connected together in both views since there is no low-rank constraint on the view graph matrix. Figure 3 (c) and (f) show the learned graphs for the two views, where the lines are produced from the learned unified matrix $U$. The final clusters are separated well. The weak connections are cut and the strong connections are strengthened by fusing the complementary information contained in two views. For the Three-Circle data set, we set the number of anchor points $k=3$ and the number of anchor neighbors $t=40$. Similar to Figure 3, the learned graphs for the two views are shown in 


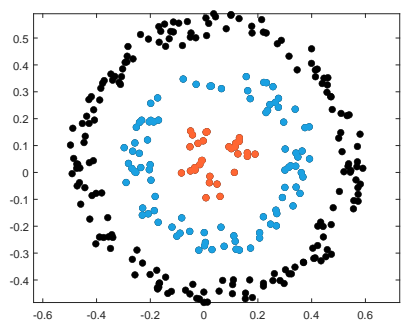

(a)

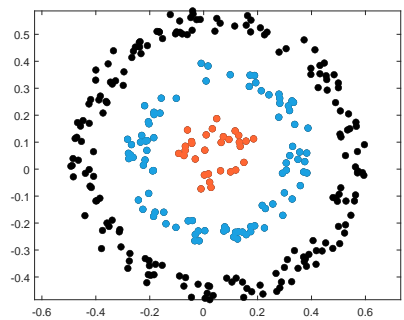

(d)

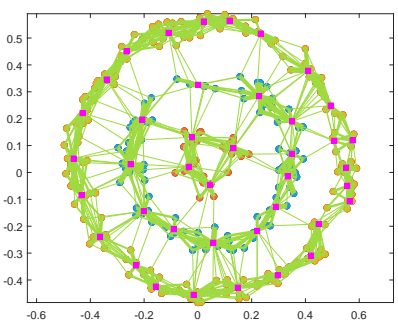

(b)

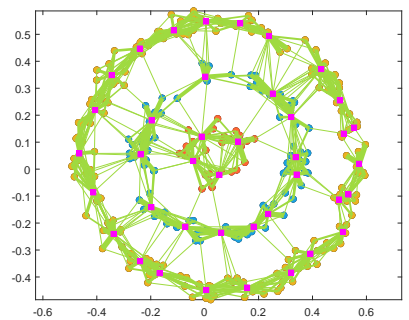

(e)

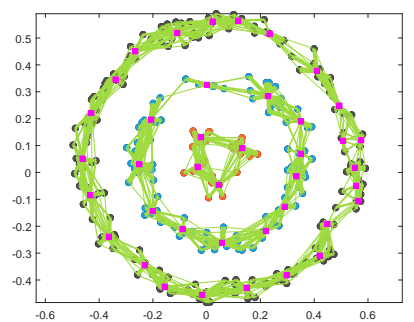

(c)

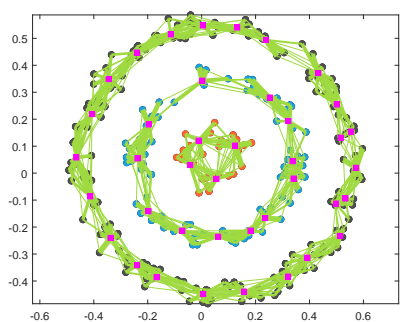

(f)

Figure 4. Clustering results on Three-Circle data set. The upper row includes the original first view data, the learned graph with the learned $S^{1}$, and the learned graph with the learned $U$. The lower row contains the original second view data, the learned graph with the learned $S^{2}$, and the learned graph with the learned $U$. The red, blue, and black dots are cluster 1 , cluster 2, and cluster 3 . The pink squares are the learned anchor points, and the green lines are the learned connections between data points and anchor points.

Figure 4 (b) and (e) with the lines from the learned $S^{1}$ and $S^{2}$. The three clusters are connected together, where the connections are closer than that in the TwoMoon data set. It is harder to separate the clusters correctly for the Three-Circle data set. From the results in Figure 4 (c) and (f), they are separated well based on the unified matrix $U$ by considering the information in two views. Additionally, it is noticed that the number of anchor points in each cluster can be learned adaptively without being specified, and the learned locations of them are well distributed.

\subsubsection{Experiments on Real-world Datasets}

To further assess the effectiveness of our proposed BIGMC, we compare BIGMC with several baselines on real-world datasets. 
Table 1. Statistics of experimental data sets

\begin{tabular}{cccccccccc}
\hline Datasets & $\mathrm{n}$ & $\mathrm{m}$ & $\mathrm{c}$ & $d^{1}$ & $d^{2}$ & $d^{3}$ & $d^{4}$ & $d^{5}$ & $d^{6}$ \\
\hline 3sources & 169 & 3 & 6 & 3560 & 3631 & 3068 & - & - & - \\
100leaves & 1600 & 3 & 100 & 64 & 64 & 64 & - & - & - \\
Caltech-7 & 1474 & 6 & 7 & 48 & 40 & 254 & 1984 & 512 & 928 \\
Caltech-20 & 2386 & 6 & 20 & 48 & 40 & 254 & 1984 & 512 & 928 \\
Mfeat & 2000 & 6 & 10 & 216 & 76 & 64 & 6 & 240 & 47 \\
WebKB & 203 & 3 & 4 & 1703 & 230 & 230 & - & - & - \\
YaleB & 650 & 3 & 10 & 2500 & 3304 & 6750 & - & - & - \\
\hline
\end{tabular}

Data sets. The following data sets are widely used in the literature.

1) 3sources ${ }^{1}$ : There are 3 views from BBC, Reuters, and Guardian. Each view has 169 news, which can be grouped into 6 clusters.

2) 100leaves $^{2}$ : There are 3 views, where each view has 1600 data points from each of 100 plant species leaves. Each object can be described by shape descriptor, fine-scale margin, and texture histogram in the 3 views, respectively.

3) Caltech-73: It is a subset of Caltech-101 data set, consisting of 6 views. Each view has 1474 images, which can be grouped into 7 clusters, i.e., faces, motorbikes, dollar bill, Garfield, stop sign, and windsor chair.

4) Caltech-204: It is also a subset of Caltech-101 data set, consisting of the same 6 views with Caltech- 7 . Each view has 2386 images, which can be partitioned into 20 classes.

5) $M f e a t^{5}$ : It is the Mfeat handwritten digit data set including handwritten digits (0-9) from the UCI repository. There are 6 views. Each view has 2000 samples and each sample can be represented by 6 types of features.

\footnotetext{
${ }^{1} \mathrm{http}: / / \mathrm{mlg}$. ucd.ie/datasets/3sources.html

${ }^{2}$ https://archive.ics.uci.edu/ml/datasets/One-hundred+plant+ species+leaves+data+set

${ }^{3}$ http://www.vision.caltech.edu/Image_Datasets/Caltech101

${ }^{4}$ http://www.vision.caltech.edu/Image_Datasets/Caltech101

${ }^{5}$ http://archive.ics.uci.edu/ml/datasets/Multiple+Features
} 
6) $W e b K B^{6}$ : There are 3 views, in which each view has 203 web-pages and 4 classes. Each web-page can be described by the anchor text of the hyper-like, the content of the page, and the title.

7) Yale $B^{7}$ : It is a subset of the extended Yale-B data set, i.e., the first 10 classes data. There are 3 views, where each view has 650 face images.

The statistical information of all these data sets is shown in Table $1 . n$ is the number of data objects. $m$ is the number of views. $c$ is the required number of clusters. $d^{v}$ indicates the dimension of features in $v$-th view.

Baselines. The following baseline methods are compared with our proposed BIGMC methods.

1) Multi-View Clustering via Concept factorization (MVCC) [50]: Incorporating the local manifold regularization into concept factorization to drive a common representation for multiple views.

2) Pairwise Multi-view Low-Rank Sparse Subspace Clustering (P-MVLRSSC) [51]:

Performing multi-view clustering based on low-rank representation and sparse subspace learning between affinity matrices of the pairs of views.

3) Centroid Multi-view Low-Rank Sparse Subspace Clustering (C-MVLRSSC) [51]: Performing multi-view clustering based on low-rank representation and sparse subspace learning between affinity matrices towards a common centroid.

4) Graph-based Multi-view Clustering (GMC) [38]: Constructing the graph of each view based on the pairwise similarity between any two data samples and fusing them to produce a unified matrix. The final clusters can be obtained from the unified matrix.

5) Multi-View Graph Learning (MVGL) [19]: Learning the initial graph of each view, optimizing it with a rank constraint on the Laplacian matrix, and integrating

\footnotetext{
${ }^{6}$ https://linqs.soe.ucsc.edu/data

${ }^{7}$ http://vision.ucsd.edu/ leekc/ExtYaleDatabase/ExtYaleB.html
} 
the optimized graphs into a global graph.

6) Multi-view Spectral Clustering (MVSC) [39]: Learning a bipartite graph for each view, combining them using a local manifold fusion method, and running spectral clustering on the fused graph.

7) Multi-view Learning with Adaptive Neighbours (MLAN) [18]: Performing clustering and local structure learning simultaneously and obtaining an optimal graph without fusion.

In the baselines, the state-of-the-art comparisons, MVCC, P-MVLRSSC, and C-MVLRSSC methods are based on subspace learning. GMC, MVGL, and MLAN methods are graph-based multi-view clustering. MVSC method is a bipartite graph-based multi-view clustering.

Experiment Settings. For the comparisons, we downloaded the source codes from the authors' websites and followed the experimental setting as well as the parameter tuning steps of their papers. All the baselines and our proposed method are implemented in the Matlab development environment. For BIGMC, we empirically set $t=n / 5$ and $k=5$. The initial value of parameter $\beta$ is set to 1 . Its value is adaptively tuned in the optimization procedure of the objective function for each data set. Six common metrics are utilized to evaluate the clustering performance: the accuracy (ACC), the normalized mutual information (NMI), the adjusted rand index (ARI), the F-measure (F-M), the precision (PRE), and the recall (REC). To randomize the experiments, each method is run 5 times $[52,53]$ and the means, as well as standard deviations of the metrics, are reported.

Results. Table 2 shows the clustering results with the six metrics by different methods on the seven real-world datasets. We highlight the best results in bold. From the table, it can be noticed that our proposed BIGMC approach acquires better performance than the baselines. 
Table 2. Clustering results (mean \pm standard-deviation) with metrics (ACC, NMI, ARI, F-M, PRE, and REC) by different methods on 7 real-world datasets

\begin{tabular}{|c|c|c|c|c|c|c|c|c|c|}
\hline Metrics & Methods & 3sources & 100leaves & Caltech-7 & Caltech-20 & Mfeat & WebKB & YaleB & Ave \\
\hline \multirow{5}{*}{$\mathrm{ACC}$} & P-MLRSSC & $0.682 \pm 0.05$ & $0.030 \pm 0.00$ & $0.609 \pm 0.08$ & $0.434 \pm 0.02$ & $0.592 \pm 0.04$ & $0.425 \pm 0.03$ & $0.481 \pm 0.08$ & $0.465 \pm 0.04$ \\
\hline & GMC & $0.692 \pm 0.00$ & $0.824 \pm 0.00$ & $0.692 \pm 0.00$ & $0.456 \pm 0.00$ & $0.882 \pm 0.00$ & $0.769 \pm 0.00$ & $0.434 \pm 0.00$ & $0.678 \pm 0.00$ \\
\hline & MVGL & $0.302 \pm 0.00$ & $0.766 \pm 0.00$ & $0.579 \pm 0.00$ & $0.578 \pm 0.00$ & $0.856 \pm 0.00$ & $0.581 \pm 0.00$ & $0.300 \pm 0.00$ & $0.566 \pm 0.00$ \\
\hline & MVSC & $0.531 \pm 0.00$ & $0.717 \pm 0.00$ & $0.621 \pm 0.00$ & $0.575 \pm 0.00$ & $0.703 \pm 0.00$ & $0.567 \pm 0.00$ & $0.468 \pm 0.00$ & $0.597 \pm 0.00$ \\
\hline & BIGMC & $0.797 \pm 0.00$ & $0.921 \pm 0.00$ & $0.785 \pm 0.00$ & $0.611 \pm 0.00$ & $0.932 \pm 0.01$ & $0.795 \pm 0.00$ & $0.575 \pm 0.01$ & $0.774 \pm 0.00$ \\
\hline \multirow[t]{5}{*}{ NMI } & MVCC & $0.698 \pm 0.01$ & $0.552 \pm 0.00$ & $0.464 \pm 0.00$ & $0.564 \pm 0.00$ & $0.422 \pm 0.00$ & $0.418 \pm 0.00$ & $0.088 \pm 0.00$ & $0.458 \pm 0.00$ \\
\hline & P-MLRSSC & $0.594 \pm 0.03$ & $0.442 \pm 0.01$ & $0.500 \pm 0.02$ & $0.487 \pm 0.01$ & $0.700 \pm 0.02$ & $0.355 \pm 0.03$ & $0.378 \pm 0.04$ & $0.493 \pm 0.02$ \\
\hline & C-MLRSSC & $0.595 \pm 0.03$ & $0.440 \pm 0.01$ & $0.497 \pm 0.03$ & $0.477 \pm 0.01$ & $0.703 \pm 0.00$ & $0.376 \pm 0.03$ & $0.399 \pm 0.02$ & $0.498 \pm 0.02$ \\
\hline & GMC & $0.622 \pm 0.00$ & $0.929 \pm 0.00$ & $0.660 \pm 0.00$ & $0.481 \pm 0.00$ & $0.905 \pm 0.00$ & $0.435 \pm 0.00$ & $0.449 \pm 0.00$ & $0.640 \pm 0.00$ \\
\hline & BIGMC & $0.705 \pm 0.00$ & $0.960 \pm 0.01$ & $0.697 \pm 0.00$ & $0.624 \pm 0.00$ & $0.910 \pm 0.00$ & $0.540 \pm 0.00$ & $0.525 \pm 0.01$ & $0.709 \pm 0.00$ \\
\hline \multirow[t]{8}{*}{ ARI } & MVCC & $0.631 \pm 0.00$ & $0.121 \pm 0.00$ & $0.298 \pm 0.00$ & $0.487 \pm 0.00$ & $0.255 \pm 0.00$ & $0.468 \pm 0.00$ & $0.028 \pm 0.00$ & $0.329 \pm 0.00$ \\
\hline & P-MLRSSC & $0.565 \pm 0.06$ & $0.060 \pm 0.00$ & $0.324 \pm 0.02$ & $0.349 \pm 0.05$ & $0.548 \pm 0.03$ & $0.246 \pm 0.03$ & $0.200 \pm 0.02$ & $0.327 \pm 0.00$ \\
\hline & C-MLRSSC & $0.557 \pm 0.08$ & $0.059 \pm 0.00$ & $0.334 \pm 0.03$ & $0.343 \pm 0.06$ & $0.559 \pm 0.00$ & $0.266 \pm 0.03$ & $0.222 \pm 0.01$ & $0.334 \pm 0.03$ \\
\hline & GMC & $0.443 \pm 0.00$ & $0.497 \pm 0.00$ & $0.594 \pm 0.00$ & $0.128 \pm 0.00$ & $0.850 \pm 0.00$ & $0.440 \pm 0.00$ & $0.157 \pm 0.00$ & $0.444 \pm 0.00$ \\
\hline & MVGL & $-0.036 \pm 0.00$ & $0.506 \pm 0.00$ & $0.395 \pm 0.00$ & $0.263 \pm 0.00$ & $0.832 \pm 0.00$ & $0.083 \pm 0.00$ & $0.093 \pm 0.00$ & $0.305 \pm 0.00$ \\
\hline & MVSC & $0.426 \pm 0.00$ & $0.318 \pm 0.00$ & $0.436 \pm 0.00$ & $0.260 \pm 0.00$ & $0.694 \pm 0.00$ & $0.068 \pm 0.00$ & $0.147 \pm 0.00$ & $0.336 \pm 0.00$ \\
\hline & MLAN & $0.571 \pm 0.00$ & $0.818 \pm 0.01$ & $0.572 \pm 0.00$ & $0.197 \pm 0.01$ & $0.940 \pm 0.00$ & $0.373 \pm 0.00$ & $0.090 \pm 0.00$ & $0.509 \pm 0.00$ \\
\hline & BIGMC & $0.661 \pm 0.00$ & $0.883 \pm 0.01$ & $0.690 \pm 0.00$ & $0.498 \pm 0.01$ & $0.940 \pm 0.01$ & $0.546 \pm 0.00$ & $0.244 \pm 0.02$ & $0.615 \pm 0.01$ \\
\hline \multirow[t]{2}{*}{ F-M } & & & $0.136 \pm 0.00$ & & & $0.332 \pm 0.00$ & $0.664 \pm 0.00$ & $0.148 \pm 0.00$ & $0.436 \pm 0.00$ \\
\hline & BIGMC & $0.751 \pm 0.00$ & $0.882 \pm 0.01$ & $0.797 \pm 0.00$ & $0.557 \pm 0.00$ & $0.956 \pm 0.00$ & $0.753 \pm 0.00$ & $0.350 \pm 0.02$ & $0.704 \pm 0.00$ \\
\hline \multirow[t]{8}{*}{ PRE } & MVCC & $0.613 \pm 0.00$ & $0.076 \pm 0.00$ & $0.759 \pm 0.00$ & $0.561 \pm 0.00$ & $0.322 \pm 0.00$ & $0.708 \pm 0.00$ & $0.118 \pm 0.00$ & $0.461 \pm 0.00$ \\
\hline & P-MLRSSC & $0.707 \pm 0.05$ & $0.040 \pm 0.00$ & $0.697 \pm 0.04$ & $0.426 \pm 0.05$ & $0.473 \pm 0.03$ & $0.663 \pm 0.04$ & $0.234 \pm 0.02$ & $0.463 \pm 0.03$ \\
\hline & C-MLRSSC & $0.696 \pm 0.05$ & $0.040 \pm 0.00$ & $0.711 \pm 0.05$ & $0.425 \pm 0.05$ & $0.484 \pm 0.00$ & $0.682 \pm 0.04$ & $0.249 \pm 0.01$ & $0.470 \pm 0.03$ \\
\hline & GMC & $0.484 \pm 0.00$ & $0.352 \pm 0.00$ & $0.886 \pm 0.00$ & $0.228 \pm 0.00$ & $0.826 \pm 0.00$ & $0.592 \pm 0.00$ & $0.204 \pm 0.00$ & $0.510 \pm 0.00$ \\
\hline & MVGL & $0.218 \pm 0.00$ & $0.380 \pm 0.00$ & $0.762 \pm 0.00$ & $0.327 \pm 0.00$ & $0.789 \pm 0.00$ & $0.423 \pm 0.00$ & $0.164 \pm 0.00$ & $0.437 \pm 0.00$ \\
\hline & MVSC & $0.529 \pm 0.00$ & $0.205 \pm 0.00$ & $0.667 \pm 0.00$ & $0.325 \pm 0.00$ & $0.651 \pm 0.00$ & $0.417 \pm 0.00$ & $0.193 \pm 0.00$ & $0.427 \pm 0.00$ \\
\hline & MLAN & $0.609 \pm 0.00$ & $0.775 \pm 0.01$ & $0.739 \pm 0.00$ & $0.279 \pm 0.00$ & $0.945 \pm 0.00$ & $0.559 \pm 0.00$ & $0.157 \pm 0.00$ & $0.580 \pm 0.00$ \\
\hline & BIGMC & $0.718 \pm 0.00$ & $0.870 \pm 0.02$ & $0.904 \pm 0.00$ & $0.576 \pm 0.01$ & $0.953 \pm 0.00$ & $0.742 \pm 0.01$ & $0.268 \pm 0.01$ & $0.688 \pm 0.01$ \\
\hline \multirow[t]{8}{*}{ REC } & MVCC & $0.823 \pm 0.00$ & $0.653 \pm 0.00$ & $0.334 \pm 0.00$ & $0.530 \pm 0.00$ & $0.342 \pm 0.00$ & $0.626 \pm 0.00$ & $0.197 \pm 0.00$ & $0.515 \pm 0.00$ \\
\hline & P-MLRSSC & $0.619 \pm 0.06$ & $0.771 \pm 0.01$ & $0.414 \pm 0.03$ & $0.512 \pm 0.04$ & $0.843 \pm 0.02$ & $0.337 \pm 0.03$ & $0.430 \pm 0.02$ & $0.561 \pm 0.03$ \\
\hline & C-MLRSSC & $0.619 \pm 0.07$ & $0.770 \pm 0.02$ & $0.416 \pm 0.02$ & $0.503 \pm 0.05$ & $0.843 \pm 0.00$ & $0.350 \pm 0.03$ & $0.455 \pm 0.02$ & $0.565 \pm 0.03$ \\
\hline & GMC & $0.805 \pm 0.00$ & $0.887 \pm 0.00$ & $0.609 \pm 0.00$ & $0.673 \pm 0.00$ & $0.909 \pm 0.00$ & $0.858 \pm 0.00$ & $0.378 \pm 0.00$ & $0.731 \pm 0.00$ \\
\hline & MVGL & $0.768 \pm 0.00$ & $0.789 \pm 0.00$ & $0.455 \pm 0.00$ & $0.567 \pm 0.00$ & $0.920 \pm 0.00$ & $0.858 \pm 0.00$ & $0.270 \pm 0.00$ & $0.647 \pm 0.00$ \\
\hline & MVSC & $0.628 \pm 0.00$ & $0.826 \pm 0.00$ & $0.629 \pm 0.00$ & $0.567 \pm 0.00$ & $0.828 \pm 0.00$ & $0.873 \pm 0.00$ & $0.405 \pm 0.00$ & $0.679 \pm 0.00$ \\
\hline & MLAN & $0.777 \pm 0.00$ & $0.869 \pm 0.00$ & $0.734 \pm 0.00$ & $0.557 \pm 0.02$ & $0.947 \pm 0.00$ & $0.831 \pm 0.00$ & $0.321 \pm 0.00$ & $0.719 \pm 0.00$ \\
\hline & BIGMC & $0.834 \pm 0.00$ & $0.893 \pm 0.01$ & $0.738 \pm 0.00$ & $0.698 \pm 0.00$ & $0.966 \pm 0.00$ & $0.914 \pm 0.01$ & $0.495 \pm 0.02$ & $0.773 \pm 0.00$ \\
\hline
\end{tabular}

In terms of $\mathrm{ACC}$, our proposed BIGMC method achieves the best performance

for 6 out of 7 datasets. For the Mfeat dataset, BIGMC also finishes the second

and performs better than the other methods by a large margin except of the 
MLAN method. In terms of NMI, BIGMC gives a better performance than the comparisons for 6 out of 7 datasets. Moreover, it also achieves the second-best performance on Mfeat dataset. We can also see that BIGMC performs better for 6 out of 7 datasets in terms of ARI and has comparable performance with the MLAN method. In terms of F-M, PRE, and REC metrics, BIGMC is markedly better than all the baselines on all datasets. To be more specific, BIGMC has a smaller deviation than the P-MLRSSC method although they achieve the same average PRE values. Note that the average metric value for each method on all datasets can be seen in the last column. BIGMC, on average, outperforms all the other compared methods.

BIGMC can learn better anchor points based on the learned unified graph. MVCC drives a common consensus representation through manifold regularization and concept factorization. One reason why it is worse than BIGMC is that the constructed Laplacian matrices are fixed during the learning process. BIGMC performs better than P-MVLRSSC and C-MVLRSSC, both of which rely on the additional K-means clustering method.

Compared with the graph-based methods, i.e., GMC, MVGL, MVSC, and MLAN, BIGMC has a superior performance since it can learn a better unified graph by learning the individual graph, the unified graph, and the consensus anchor points across all views simultaneously.

To further demonstrate the adaptive ability of learning anchor points, we give an example to show the learning results of anchors by our method on 3 real-world datasets including 3sources, Caltech-7, and WebKB. The ground truth number of samples, the predicted number of samples, and the predicted number of anchors for each class are shown in Figure 5. From the figure, we can observe that the predicted number of anchors is much smaller than that of samples for each dataset. 


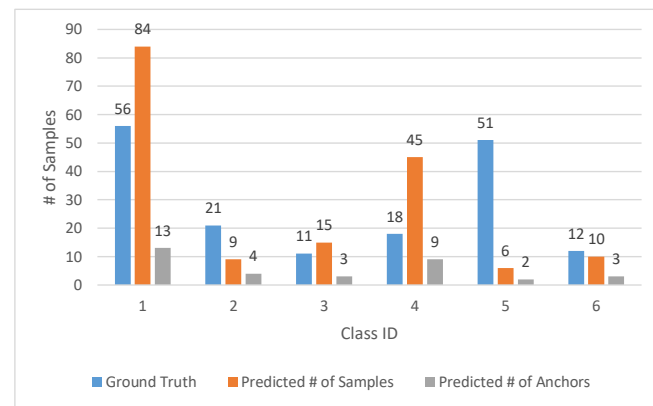

(a)

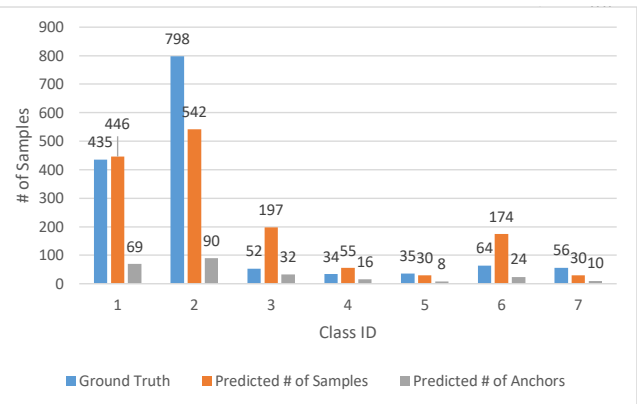

(b)

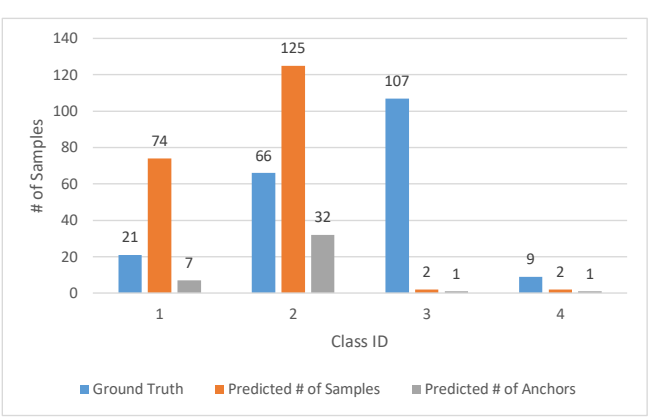

(c)

Figure 5. The learning results of anchors by BIGMC on 3 real-world datasets compared with the ground truth and the predicted number of samples in each class. (a) 3sources; (b) Caltech-7; (c) WebKB.

For different classes, the number of anchors can be learned adaptively, and more anchors can be learned for a class with a larger number of samples.

Mode Evaluation. To further show the effectiveness of our proposed BIGMC, we evaluate 4 variants of BIGMC as follow:

1) BIGMC_A: The learned unified graph matrix $U$ are not used to improve the initialized anchors $A$ for each view by removing Steps 6 and 14 in Algorithm 1.

2) BIGMC_AS: The learned unified graph matrix $U$ are not employed to improve both the initialized anchors $A$ and the initialized similarity matrix $S$ for each view by removing Steps 6, 8, and 14 in Algorithm 1.

3) BIGMC_W: The weight of each view $\delta$ is set to $1 / m$ by removing Step 9 in Algorithm 1.

4) BIGMC_K: The learned partition matrix $F_{1}$ for data samples is as the input of 


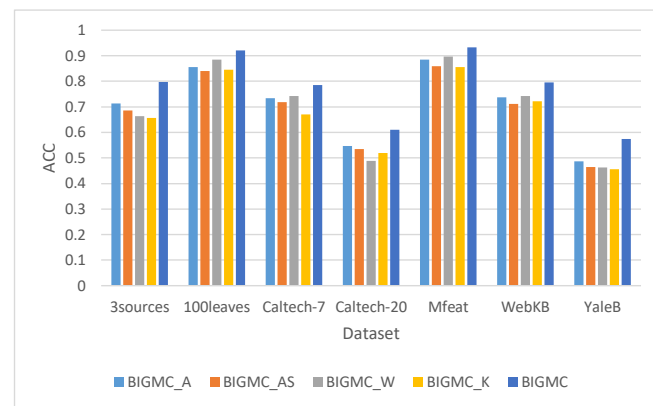

(a)

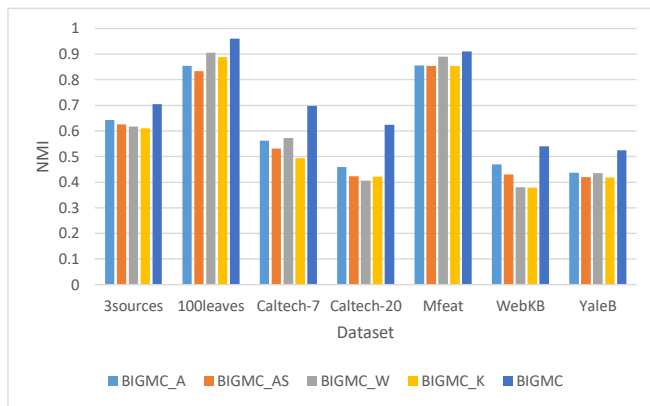

(b)

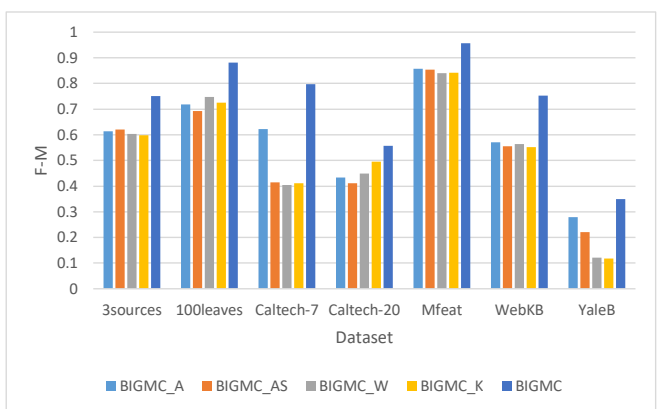

(c)

Figure 6. Clustering performance comparison of BIGMC and its 4 variants on 7 real-world datasets in terms of metrics (ACC, NMI, and F-M).

the additional clustering method, i.e., K-means, to generate the final clusters. In this case, the reason why we use $F_{1}$ not $F$ is that $F$ as a block matrix includes $F_{1}$ (the partition matrix of data samples) and $F_{2}$ (the partition matrix of anchors).

Figure 6 shows the clustering performance of BIGMC and its 4 variants on 7 real-world datasets. Figure $6(\mathrm{a}),(\mathrm{b})$, and (c) present the performance in terms of ACC, NMI, and F_M, respectively. It can be noted that BIGMC has a better performance than the four variants. This indicates that each component of BIGMC is essential and they can help each other to improve the performance. Specifically, comparing the performance of BIGMC_A and BIGMC_AS, BIGMC_A outperforms BIGMC_AS since the earned unified graph matrix $U$ goes back to improve the initialized similarity matrix $S$. This also shows the effectiveness of the joint learning strategy. 


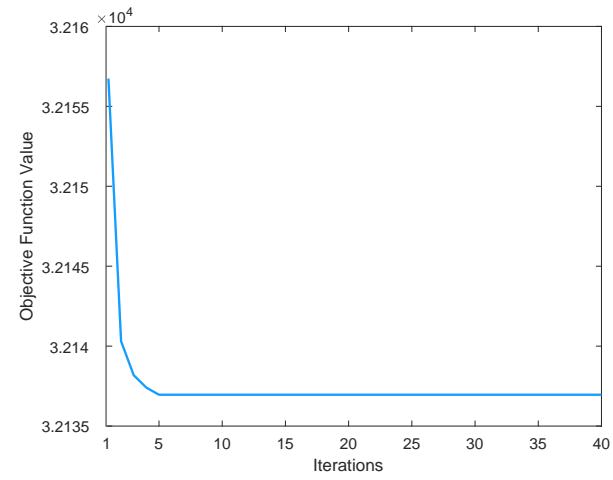

(a)

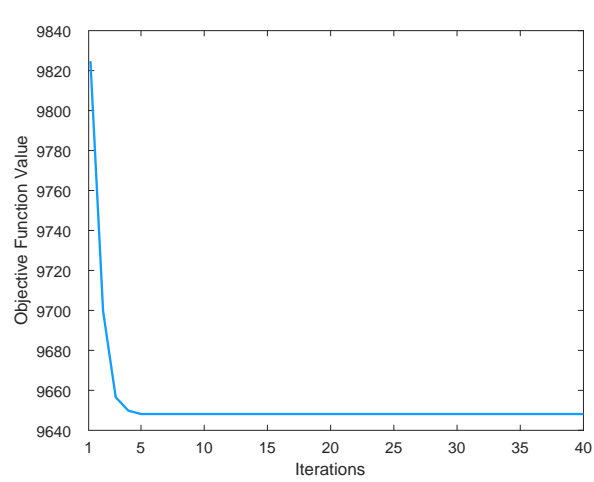

(c)

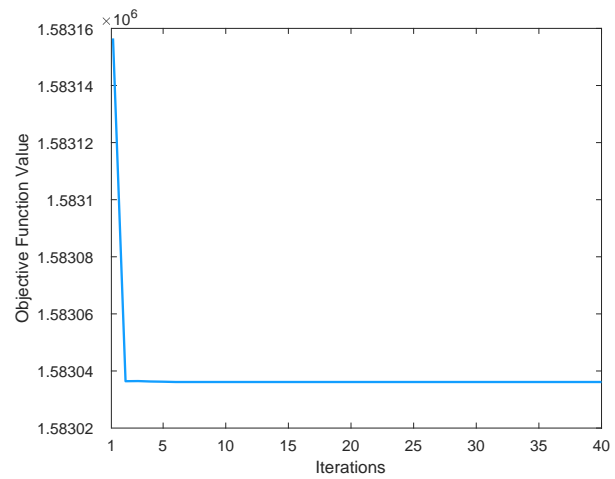

(b)

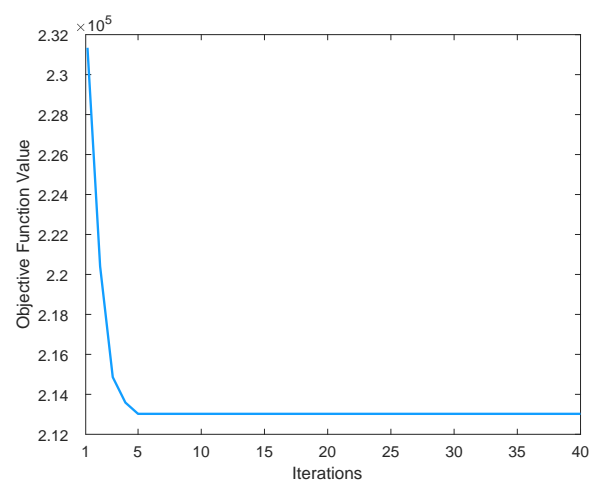

(d)

Figure 7. Convergence curves over different datasets. (a) 100leaves. (b) Caltech20. (c) WebKB. (d) YaleB.

Convergence Study. To show the effectiveness of the used optimization strategy for the objective function of BIGMC method, we plot the convergence curves of BIGMC over 4 different datasets in Figure 7. For each sub-figure, the $x$ axis denotes the number of iterations and the $y$-axis denotes the objective function value. It can be noticed that BIGMC converges quickly for all datasets. To be more specific, it converges within 5 iterations on 100leaves, Caltech-20, WebKB, and YaleB datasets. This indicates that we presented an efficient optimized solution.

Running Time Comparison. The effectiveness of our proposed BIGMC method has been evaluated by all the above experiments. In this section, we aim to explore the efficiency of BIGMC and compare it to that of the state-of-the-art 
methods. To exclude the influence of initialization, all the algorithms are conducted 5 times and the mean values are shown in Table 3 . It can be observed that the MVSC method performs the best and BIGMC performs the second on average. Moreover, MVSC, BIGMC, MLAN, and GMC have comparable performance.

Table 3. Averaged running time by different methods on 7 real-world datasets (in second)

\begin{tabular}{ccccccccc}
\hline Methods & 3sources & 100leaves & Caltech-7 & Caltech-20 & Mfeat & WebKB & YaleB & Ave \\
\hline MVCC & 19.672 & 107.170 & 123.957 & 259.248 & 167.966 & 6.354 & 134.167 & 116.933 \\
P-MLRSSC & 1.016 & 6.288 & 155.300 & 621.127 & 370.181 & 0.470 & 24.193 & 168.368 \\
C-MLRSSC & 0.713 & 29.177 & 158.439 & 679.249 & 384.128 & 0.579 & 25.309 & 182.513 \\
GMC & 0.916 & 16.143 & 8.472 & 21.879 & 53.744 & 1.087 & 1.984 & 14.889 \\
MVGL & 0.634 & 74.010 & 169.243 & 611.509 & 497.051 & 0.969 & 9.092 & 194.644 \\
MVSC & $\mathbf{0 . 2 0 6}$ & $\mathbf{4 . 5 4 5}$ & $\mathbf{3 . 0 4 4}$ & $\underline{12.250}$ & $\mathbf{9 . 4 4 7}$ & $\mathbf{0 . 1 2 1}$ & $\mathbf{0 . 3 7 9}$ & $\mathbf{4 . 2 8 5}$ \\
MLAN & 0.236 & 7.222 & 12.862 & 39.641 & 18.223 & $\underline{0.155}$ & $\underline{2.037}$ & 11.482 \\
BIGMC & $\underline{0.208}$ & $\underline{5.376}$ & $\underline{6.086}$ & $\mathbf{1 1 . 4 2 1}$ & $\underline{15.502}$ & 0.288 & 2.350 & $\underline{5.890}$ \\
\hline
\end{tabular}

\subsection{Chapter Summary}

In this chapter, we proposed a novel bipartite graph based multi-view clustering (BIGMC) approach. BIGMC jointly learns the similarity graph of each view, the unified bipartite graph, and the representative uniform anchor set in a framework. Moreover, BIGMC adaptively determines the importance of each view and directly obtains the final clusters with a low-rank constraint, which is imposed on the unified bipartite Laplacian matrix. Finally, the consensus information is uncovered and the clustering structures are learned through an alternating optimization strategy. The experiments on synthetic and real-world datasets are conducted to demonstrate the effectiveness of BIGMC. In addition, obtaining a globally optimum solution of the objective function is considered as an open problem for our future work. 


\section{CHAPTER 3}

\section{Incomplete Multi-view Clustering}

\subsection{Background}

Incomplete multi-view clustering (IMC) needs not only to make full use of the complementary information of all views, but also to eliminate the negative impacts of missing samples. Existing IMC methods are mostly based on subspace learning [54], kernel learning [55], and spectral clustering [56]. According to different handling of incomplete views, they can be roughly divided into two categories. The first category concentrates on employing an imputation technique to fill the incomplete views. Most filling methods complete the incomplete views with average feature values. For example, Zhou et al. [56] propose a graph-based IMC (GIMC) method based on mean value filling. As a "two-stage" algorithm, GIMC first fills the missing samples of each incomplete view with the average sample features in those views. Then it constructs a complete graph for each view on the completed views, gives weights for them, and fuses them into a consensus graph. However, such filling methods may introduce some useless even noisy information, resulting in the poor quality of constructed graphs. Some advanced filling methods are proposed to perform matrix imputation. For instance, two multi-kernel k-means methods with incomplete kernels are presented in [55]. They jointly impute incomplete kernel matrices and combine them to perform clustering, which is termed as a "one-stage" algorithm. Although these two methods perform well but depend too much on kernel learning.

The second category aims to directly learn a common representation or subspace without imputation. For example, partial multi-view clustering (PVC) [57] utilizes the technique of non-negative matrix factorization (NMF) to solve the incomplete problem of views. Specifically, PVC directly learns a common subspace 
for aligned samples in two views and a private latent representation for unaligned samples. Inspired by PVC, Zhao et al. [58] propose an incomplete multi-modality grouping (IMG) approach. IMG combines PVC and manifold learning to learn a latent representation of two views. Both PVC and IMG are designed for the incomplete two-view problems, and thus have limited ability to handle incomplete multi-view problems. Hu et al. [59] propose a doubly aligned incomplete multiview clustering (DAIMC) method. It first learns a common latent feature matrix for all views using the given sample alignment information, and then builds a consensus basis matrix to reduce the influence of missing samples.

By observations, although some efforts have been made for IMC, there are still some challenges to overcome. First, the filling methods have significant impacts on the clustering, especially for data with a large missing ratio. No matter performing imputation is to incomplete features, similarity matrix, or clustering matrix, the contained useless even noisy information would lead to poor quality of the imputation. As a result, the poor imputation affects the clustering of all the missing and un-missing samples. Second, some methods only work for incomplete two-view data and fail to deal with incomplete multi-view data. e.g., PVC and IMG. Third, both NMF and subspace learning-based IMC focus on learning the consensus representation without considering the geometric structures of data. In the meanwhile, although certain graph-based IMC methods can effectively exploit the data structures but cannot learn the global optimal consensus representation because of the two-stage strategy.

To address the above issues, we propose a novel joint partition and graph (JPG) learning approach for IMC [60]. Our proposed JPG method aims to jointly construct local incomplete graph matrices, generate incomplete base partition matrices, stretch them to produce a unified partition matrix, and employ it to learn 


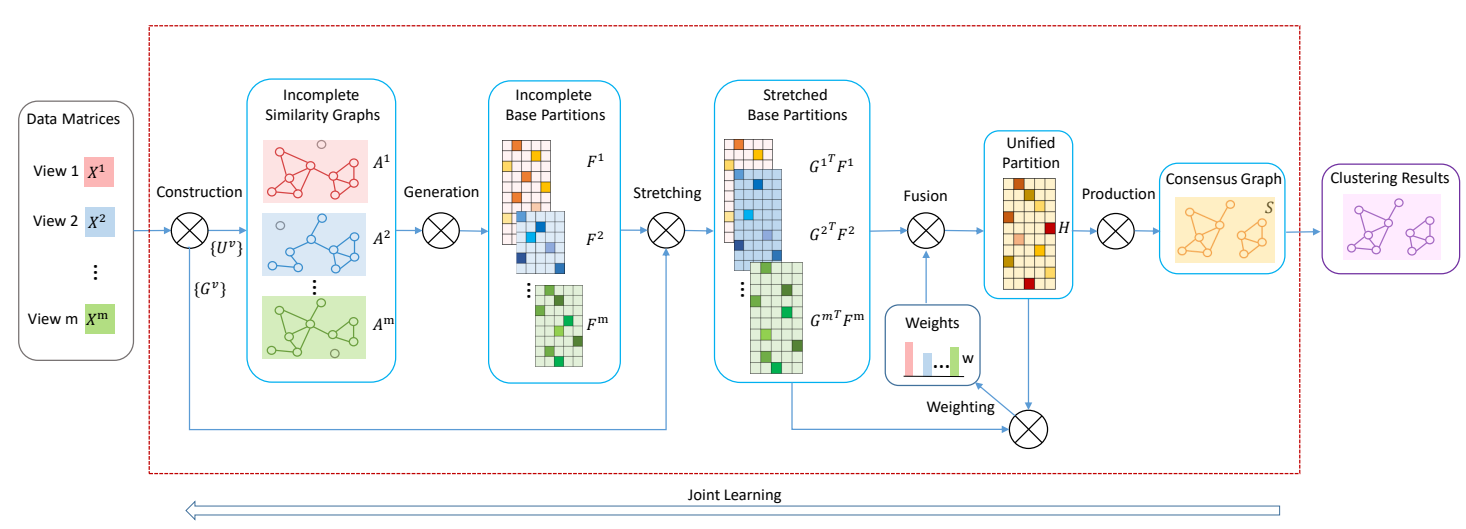

Figure 8. The framework of our proposed JPG method.

a consensus graph matrix. The overall flow of JPG is shown in Figure 8. To be more specific, the five sub-tasks can be achieved in a unified framework. We first construct an incomplete similarity graph matrix $A^{v}$ for each view. This sub-task is performed only based on the un-missing samples $U^{v}$ to guarantee the utilized information authenticity. The grey cycles in the figure represent the missing samples. There are no connections between missing samples and un-missing samples. Second, the incomplete base partition matrix $F^{v}$ for each view is generated in the guide of the corresponding local graph matrix $A^{v}$. This sub-task is to obtain the information contained in partitions. Next, all base partition matrices are stretched with index matrices $\left\{G^{v}\right\}$ to produce a unified partition matrix $H$. Different partition matrices have different sizes. The unified partition matrix contains complementary information from all views. It can be also noted that JPG can weigh each view automatically $\left(w_{v}\right)$ based on the discrepancy between the view matrix and the unified matrix. Then we employ the unified partition matrix to learn a consensus graph matrix $S$. A rank constraint is added on all Laplacian matrices $\left\{L_{a}^{v}\right\}$ and $L_{s}$ to ensure that there are required exact $c$ connected components in the graph matrices. The final clusters can be directly obtained from $S$ without any additional clustering methods. In the optimization process, these five sub-tasks 
can help each other in a mutual reinforcement manner.

In summary, the main contributions are highlighted as follows:

- We propose a novel joint partition and graph (JPG) learning approach, which can address both complete and incomplete multi-view clustering problems. In the incomplete cases, JPG can deal with the case that few samples have complete views and another case that all samples have missing views.

- JPG learns the local graph and base partition for each view, generates a unified partition, and produces a consensus graph jointly. It also determines the weight of each view automatically and generates the final clusters directly from the consensus graph.

- JPG exploits an efficient alternation optimization strategy to optimize all the variables step by step. JPG is also a parameter-free method, where all the parameters can be adaptively determined in the iterations.

The rest of this chapter is organized as follows: Section 3.2 reviews the related work on multi-view clustering methods. In Section 3.3, we present the details of our proposed method. Section 3.4 presents the optimization strategy. Section 3.5 shows experiments on real-world data sets. In Section 3.6, we provide the conclusion.

\subsection{Related Work}

Most related works to our proposed JPG method are graph-based incomplete multi-view clustering methods such as [54, 61, 56, 62]. However, existing approaches cannot simultaneously handle the limitations mentioned above. For example, Wang et al. [62] propose a perturbation-oriented incomplete multi-view clustering (PIC) method. PIC utilizes a two-stage strategy, where it first completes the learned similarity graph matrix for each view with the average similarity values 
of the available views, and then learns a consensus Laplacian matrix by minimizing perturbation bounds. However, this method cannot learn the global optimal consensus representation since it disconnects the processes of imputation and clustering. For [56], as we mentioned above, the filling and two-strategy strategies would lead to the poor quality of graphs. To this end, one-stage methods for graph-based incomplete multi-view clustering are studied in [54,61]. Wen et al. [54] first construct a graph for each view based on subspace learning, generate a partition for each view, and fuse the obtained partition into a unified matrix. They in [61] use matrix factorization technique to learn the common latent representation. Although both of them use the information from the un-missing samples, they ignore the weight of each view in the fusion and also rely on the initialization because of the used subspace learning and matrix factorization techniques. Additionally, these two methods need the K-means algorithm to get the final clusters.

Our work is also related to multi-view clustering in the partition space [63, 64, 65]. Tao Et al. [63] focus on the high-level information in the partition space and thus propose a multi-view ensemble clustering (MVEC) method to learn a consensus partition in an ensemble clustering way. However, the base partitions are pre-given and the post-processing K-means method is also required to obtain the final clusters. Kang et al. [64] design a multiple partitions aligned clustering (mPAC) method. mPAC aligns each base partition to generate a consensus partition matrix through a rotation matrix. It performs well in the experiments. While it utilizes subspace clustering technique to produce base partition and also ignores the local structure of data. The authors in [65] present a partition level multi-view subspace clustering approach, which has the same problem with [64]. All these three methods have demonstrated that integrating the information of partition space can improve clustering performance. However, it is also worth 
noting that they are all for complete multi-view clustering problems and cannot handle incomplete multi-view clustering problems.

\subsection{Proposed Method}

In this section, we first describe some notations and then present the proposed JPG method. Throughout the paper, matrices, vectors, and scalars are respectively written in italic capital (e.g., $X$ ), lowercase (e.g., x), and italic lowercase (e.g., $x$ )

letters. Given a matrix $X \in \mathbb{R}^{d \times n}, \mathrm{x}_{j} \in \mathbb{R}^{d \times 1}$ is its $j$-th column vector; $x_{i j}$ is its $(i, j)$-th entry; $\operatorname{Tr}(X)$ is the trace of $X ;\|X\|_{F}$ is the Frobenius norm of $X$. Given a vector $\mathrm{x}$, let $x_{i}$ be its $i$-th entry, $\mathrm{x}^{T}$ be its transpose, and $\|\mathrm{x}\|_{p}=\left(\sum_{i=1}^{d}\left|x_{i}\right|^{p}\right)^{1 / p}$ be its $l_{p}$-norm. Matrix $\mathbf{I}$ indicates the identity matrix, and vector $\mathbf{1}$ represents its entries are one.

Our proposed JPG consists of adaptive local partition learning, multi-view partition fusion, and consensus graph construction. In each section, the main technologies and theories are described. Overall, JPG models three main tasks into a unified framework including the learning of the adaptive local partition of each view, the learning of the fusion of partitions of all views, and the construction of the consensus graph. In the framework, each view is auto-weighted; the partition of each view, the fusion partition of all views, and the consensus graph are produced jointly; the final clusters are generated directly based on the consensus graph.

\subsubsection{Adaptive Base Partition Learning}

Before learning the base partition of each view, we first generate an authentic similarity matrix for each view. From the above analysis, the authentic similarity information contained in the un-missing samples contributes to achieving a more reliable partition and eliminating the negative impacts of the missing views. Thus, each entry in the authentic similarity matrix represents the relationship between 
any two un-missing samples. For a multi-view data set with $m$ views, we denote $X^{1}, \ldots, X^{m}$ as the data matrices and $X^{v}=\left[\mathrm{x}_{1}^{v}, \ldots, \mathrm{x}_{n}^{v}\right] \in \mathbb{R}^{d_{v} \times n}$ as the $v$-th view data with $d_{v}$ dimensions as well as $n$ data points. From these the data matrices, we extract the un-missing samples for each view, and thus obtain $U^{1}, \ldots, U^{m}$, where $U^{v}=\left[\mathrm{u}_{1}^{v}, \ldots, \mathrm{u}_{n}^{v}\right] \in \mathbb{R}^{d_{v} \times n_{v}}$ as the $v$-th view available data with $d_{v}$ dimensions as well as $n_{v}$ data points. For $U^{v}$, let $\mathrm{u}_{i}^{v}$ be the $i$-th column vector and $u_{i j}^{v}$ be the $(i, j)$ th entry. The authentic similarity matrices between any two un-missing samples can be denoted as $A^{1}, \ldots, A^{m}$, where each $A^{v} \in \mathbb{R}^{n_{v} \times n_{v}}$ can have a different size.

For the $i$-th sample $\mathrm{u}_{i}^{v}$ of $U^{v}$, it belongs to one of the $c$ classes and we can connect the $j$-th sample $\mathrm{u}_{j}^{v}$ to it with the probability $a_{i j}^{v}$. In such case, the probability $a_{i j}^{v}$ can be seen as the authentic similarity between them. In general, closer $\mathrm{u}_{i}^{v}$ and $\mathrm{u}_{j}^{v}$ are likely to have a larger $a_{i j}^{v}$. Thus $a_{i j}^{v}$ is in inverse proportion to the distance between them, i.e. $\left\|u_{i}^{v}-u_{j}^{v}\right\|_{2}^{2}$. Thus, the authentic similarity matrices for all views can be learned by solving the following problem:

$$
\begin{aligned}
& \min _{\left\{A^{v}\right\}_{v=1}^{m}} \sum_{v=1}^{m} \sum_{i, j=1}^{n_{v}}\left\|\mathrm{u}_{i}^{v}-\mathrm{u}_{j}^{v}\right\|_{2}^{2} a_{i j}^{v}+\alpha \sum_{v=1}^{m}\left\|A^{v}\right\|_{F}^{2} \\
& \text { s.t. } \forall v, a_{i i}^{v}=0, a_{i j}^{v} \geq 0, \mathbf{1}^{T} \mathrm{a}_{i}^{v}=1 .
\end{aligned}
$$

where the normalization $\mathbf{1}^{T} \mathrm{a}_{i}^{v}=1$ is considered as the sparse constraint on $A^{v}$; the second term can be seen as a regularization term; the parameter $\alpha$ is a coefficient of the regularization term. If $\alpha=0$, there will be a trivial solution for problem (40), e.g., $a_{i j}^{v}=1$. In such case, only the nearest sample $\mathrm{u}_{j}^{v}$ to $\mathrm{u}_{i}^{v}$ can be connected to $\mathrm{u}_{i}^{v}$ and the other $\left(n_{v}-2\right)$ samples cannot be connected to it. This is called hard partition. If $\alpha$ is sufficiently large, all the other $\left(n_{v}-1\right)$ samples can be connected to $\mathrm{u}_{i}^{v}$ with the same probability $1 /\left(n_{v}-1\right)$. $\alpha$ can be adaptively determined as shown in Section 3.4.

As we all know, the ideal neighbor assignments can make the similarity graph have exact $c$ connected components, which is beneficial to group data samples into 
$c$ clusters. While in general, it is very hard to achieve this target only by Eq. (40) even if we can get an optimal value of $\alpha$. In such a case, all the samples may be connected together leading to one component. To address this issue, a rank constraint on each Laplacian matrix $L_{a}^{v}$ can be introduced if the corresponding similarity matrix $A^{v}$ is non-negative as shown in the following theorem.

Theorem 3. The multiplicity of the eigenvalue 0 of the Laplacian matrix equals the number of connected components in the graph associated with the similarity matrix.

Thereinto, $L_{a}^{v}=D_{a}^{v}-\left(A^{v}+A^{v T}\right) / 2$ and the degree matrix $D_{a}^{v} \in \mathbb{R}^{n_{v} \times n_{v}}$ is a diagonal matrix, where its $i$-th diagonal entry can be defined as $\sum_{j=1}^{n_{v}}\left(a_{i j}^{v}+a_{j i}^{v}\right) / 2$. From Theorem 1, it can be known that the similarity matrix $A^{v}$ is an ideal case if $\operatorname{rank}\left(L_{a}^{v}\right)=n_{v}-c$. Therefore, we would have

$$
\begin{aligned}
& \min _{\left\{A^{v}\right\}_{v=1}^{m}} \sum_{v=1}^{m} \sum_{i, j=1}^{n_{v}}\left\|\mathrm{u}_{i}^{v}-\mathrm{u}_{j}^{v}\right\|_{2}^{2} a_{i j}^{v}+\alpha \sum_{v=1}^{m}\left\|A^{v}\right\|_{F}^{2} \\
& \text { s.t. } \forall v, a_{i i}^{v}=0, a_{i j}^{v} \geq 0, \mathbf{1}^{T} \mathrm{a}_{i}^{v}=1, \operatorname{rank}\left(L_{a}^{v}\right)=n_{v}-c .
\end{aligned}
$$

While Eq. (41) is difficult to solve with the constraint $\operatorname{rank}\left(L_{a}^{v}\right)=n_{v}-c$. We denote the $i$-th smallest eigenvalue of $L_{a}^{v}$ as $\delta_{i}\left(L_{a}^{v}\right)$. Thus $\delta_{i}\left(L_{a}^{v}\right) \geq 0$ since $L_{a}^{v}$ is positive semi-definite. In such case, the constraint $\operatorname{rank}\left(L_{a}^{v}\right)=n_{v}-c$ can be ensured if $\sum_{t=1}^{c} \delta_{i}\left(L_{a}^{v}\right)=0$. From Ky Fan's Theorem [46], we can have

$$
\sum_{t=1}^{c} \delta_{t}\left(L_{a}^{v}\right)=\min _{F^{v}, F^{v T} F^{v}=\mathbf{I}_{c}} \operatorname{Tr}\left(F^{v T} L_{a}^{v} F^{v}\right) .
$$

where $F^{v} \in \mathbb{R}^{n_{v} \times c}$ is the local partition of $U^{v}$. Plugging Eq. (42) into Eq. (41), the problem will be

$$
\begin{aligned}
& \min _{\left\{A^{v}, F^{v}\right\}_{v=1}^{m}} \sum_{v=1}^{m} \sum_{i, j=1}^{n_{v}}\left\|\mathrm{u}_{i}^{v}-\mathrm{u}_{j}^{v}\right\|_{2}^{2} a_{i j}^{v}+\alpha \sum_{v=1}^{m}\left\|A^{v}\right\|_{F}^{2}+2 \lambda_{1} \sum_{v=1}^{m} \operatorname{Tr}\left(F^{v T} L_{a}^{v} F^{v}\right) \\
& \text { s.t. } \forall v, a_{i i}^{v}=0, a_{i j}^{v} \geq 0, \mathbf{1}^{T} \mathrm{a}_{i}^{v}=1, F^{v T} F^{v}=\mathbf{I}_{c} .
\end{aligned}
$$


where $\lambda_{1}$ is a hyperparameter and can be adaptively determined as shown in Section 3.4. Here, each similarity matrix $A^{v}$ with the authentic information for each view is constructed independently since each $A^{v}$ may have different size and has no relationship with the others. This is also true for the learned partition of each view. Next, we couple each partition with a unified partition matrix.

\subsubsection{Multi-View Partition Fusion}

As mentioned above, the local partition matrices, the unified partition matrix, and the consensus graph can be learned jointly so that they help the learning of the others in a reinforcement manner. Here, we first introduce the mutual learning between the first two tasks. To be more specific, a unified partition matrix $H \in \mathbb{R}^{n \times c}$ is computed from the local partition matrices $F^{1}, F^{2}, \ldots, F^{m}$. However, we have some problems to address. The first one is that each $F^{v}$ varies in size since it only contains the information of available samples. In JPG, we can obtain the completed partition $\widehat{F}^{v} \in \mathbb{R}^{n \times c}$ from $F^{v} \in \mathbb{R}^{n_{v} \times c}$ according to the following equation

$$
\widehat{F}^{v}=G^{v T} F^{v}
$$

where $G^{v} \in \mathbb{R}^{n_{v} \times n}$ is an index matrix and can be considered as a prior. In $G^{v}$, the entries are one for the un-missing samples and zero for the missing samples. Specially, each entry can be defined as follows

$$
g_{i j}^{v}= \begin{cases}1, & \text { if } \mathrm{u}_{i} \text { is } \mathrm{x}_{j} \\ 0, & \text { otherwise }\end{cases}
$$

Once we have the completed partition matrices $\widehat{F}^{1}, \widehat{F}^{2}, \ldots, \widehat{F}^{m}$, there are the other two problems: 1) the entries of each $\widehat{F}_{i}^{v}$ are continuous; 2) there are still some discrepancies among $\widehat{F}^{1}, \widehat{F}^{2}, \ldots, \widehat{F}^{m}$ besides of the influence of missing samples since each $\widehat{F}^{v}$ is not unique in an unsupervised learning manner. Generally, there are $c$ ! different partition representations for each clustering with $c$ clusters. Hence, 
the discrepancies between each $\widehat{F}^{v}$ and the unified partition $H$ cannot be measured directly using Frobenius norm. Our aim is to discover the invariant information to align the local partitions of all views. Inspired by this, we can align each $\widehat{F}^{v} \widehat{F}^{v T}$ and $H H^{T}$ instead, where $\widehat{F}^{v} \widehat{F}^{v T}$ indicates the similarity metric of $\widehat{F}^{v}$, and $H H^{T}$ is that of $H$. It can be noted that the invariant information is contained in $\widehat{F}^{v} \widehat{F}^{v T}$ for the $c$ ! partition representations. Additionally, our proposed JPG method can autoweight each view when performing the alignment. Thus, the objective function for the multi-view partition fusion can be given

$$
\min _{H, H^{T} H=\mathbf{I}_{c}} \sum_{v=1}^{m} w_{v}\left\|H H^{T}-\widehat{F}^{v} \widehat{F}^{v T}\right\|_{F}^{2} \Rightarrow \min _{H, H^{T} H=\mathbf{I}_{c}} \sum_{v=1}^{m} w_{v}\left\|H H^{T}-G^{v T} F^{v} F^{v T} G^{v}\right\|_{F}^{2}
$$

where $w_{v}$ is the weight for the $v$-th view and can be adaptively determined shown in Section 4. Then, we combine Eq. (46) with Eq. (43) to achieve the joint learning of each similarity matrix, each partition, and the unified partition.

$$
\begin{aligned}
& \min _{\left\{A^{v}, F^{v}, w^{v}\right\}_{v=1}^{m}, H} \sum_{v=1}^{m} \sum_{i, j=1}^{n_{v}}\left\|\mathrm{u}_{i}^{v}-\mathrm{u}_{j}^{v}\right\|_{2}^{2} a_{i j}^{v}+\alpha \sum_{v=1}^{m}\left\|A^{v}\right\|_{F}^{2} \\
& +2 \lambda_{1} \sum_{v=1}^{m} \operatorname{Tr}\left(F^{v T} L_{a}^{v} F^{v}\right)+\sum_{v=1}^{m} w_{v}\left\|H H^{T}-G^{v T} F^{v} F^{v T} G^{v}\right\|_{F}^{2} \\
& \text { s.t. } \forall v, a_{i i}^{v}=0, a_{i j}^{v} \geq 0, \mathbf{1}^{T} \mathrm{a}_{i}^{v}=1, F^{v T} F^{v}=\mathbf{I}_{c}, H^{T} H=\mathbf{I}_{c} .
\end{aligned}
$$

\subsubsection{Consensus Graph Construction}

With the unified partition representation $H$, a consensus graph $S$ can be constructed on it. Here, the joint learning of the unified partition matrix and the consensus graph can be achieved. We can know that this graph $S$ is shared by all views and can be employed to produce the clustering results directly without an additional clustering approach. Here, the obtained unified partition $H$ via Eq. (47) still cannot reach this goal. We have noted that the adaptive neighbor strategy can also be applied in learning $S$ to capture the local manifold structure. Therefore, 
we calculate a consensus graph $S$ from $H$ by the following equation

$$
\min _{S} \sum_{i, j=1}^{n}\left\|\mathrm{~h}_{i}-\mathrm{h}_{j}\right\|_{2}^{2} s_{i j}+\beta\|S\|_{F}^{2} \text { s.t. } s_{i i}=0, s_{i j} \geq 0, \mathbf{1}^{T} \mathrm{~s}_{i}=1 \text {. }
$$

where $\beta$ is a hyperparameter to control the sparsity between samples and can also be adaptively determined in the Section 3.4. Note that there is no guarantee that the consensus graph $S$ is optimal through Eq. (48). To ensure that there are exact c connected components in $S$, Theorem 1 can also be used on $S$. Thus we add a rank constraint to $S$ that $\operatorname{rank}\left(L_{s}\right)=n-c$. Let $\sigma_{i}\left(L_{s}\right)$ denote the $i$-th smallest eigenvalue of $L_{s}$. Since $\sigma_{i}\left(L_{s}\right) \geq 0$, similar to Eq. (42), the constraint can be transformed as

$$
\sum_{t=1}^{c} \sigma_{t}\left(L_{s}\right)=\min _{Q \in \mathbb{R}^{n \times c}, Q^{T} Q=\mathbf{I}_{c}} \operatorname{Tr}\left(Q^{T} L_{s} Q\right) .
$$

where $Q=\left\{\mathrm{q}_{1}, \ldots, \mathrm{q}_{c}\right\} \in \mathbb{R}^{n \times c}$ is the partition matrix. Adding Eq. (49) into Eq. (48), we obtain an optimal consensus. graph $S$.

$$
\begin{aligned}
& \min _{S} \sum_{i, j=1}^{n}\left\|\mathrm{~h}_{i}-\mathrm{h}_{j}\right\|_{2}^{2} s_{i j}+\beta\|S\|_{F}^{2}+2 \lambda_{2} \operatorname{Tr}\left(Q^{T} L_{s} Q\right) \\
& \text { s.t. } s_{i i}=0, s_{i j} \geq 0, \mathbf{1}^{T} \mathrm{~s}_{i}=1, Q^{T} Q=\mathbf{I}_{c} .
\end{aligned}
$$

where $\lambda_{2}$ is a hyperparameter and can be adaptively found in the Section 3.4. As a whole, the final objective function can be obtained by combining Eq. (47) and (49).

$$
\begin{gathered}
\min _{\left\{A^{v}, F^{v}, w^{v}\right\}_{v=1}^{m}, H, S} \sum_{v=1}^{m} \sum_{i, j=1}^{n_{v}}\left\|\mathrm{u}_{i}^{v}-\mathrm{u}_{j}^{v}\right\|_{2}^{2} a_{i j}^{v}+\alpha \sum_{v=1}^{m}\left\|A^{v}\right\|_{F}^{2} \\
+2 \lambda_{1} \sum_{v=1}^{m} \operatorname{Tr}\left(F^{v T} L_{a}^{v} F^{v}\right)+\sum_{v=1}^{m} w_{v}\left\|H H^{T}-G^{v T} F^{v} F^{v T} G^{v}\right\|_{F}^{2} \\
+\sum_{i, j=1}^{n}\left\|\mathrm{~h}_{i}-\mathrm{h}_{j}\right\|_{2}^{2} s_{i j}+\beta\|S\|_{F}^{2}+2 \lambda_{2} \operatorname{Tr}\left(Q^{T} L_{s} Q\right) \\
\text { s.t. } \forall v, a_{i i}^{v}=0, a_{i j}^{v} \geq 0, \mathbf{1}^{T} \mathrm{a}_{i}^{v}=1, F^{v T} F^{v}=\mathbf{I}_{c}, \\
H^{T} H=\mathbf{I}_{c}, s_{i i}=0, s_{i j} \geq 0, \mathbf{1}^{T} \mathrm{~s}_{i}=1, Q^{T} Q=\mathbf{I}_{c} .
\end{gathered}
$$


From Eq. (51), we can see the learning of all authentic similarity matrices $\widehat{A}^{1}, \widehat{A}^{2}, \ldots, \widehat{A}^{m}$, all local partition matrices $\widehat{F}^{1}, \widehat{F}^{2}, \ldots, \widehat{F}^{m}$, the unified partition matrix $H$, and the consensus graph $S$ is coupled into a joint problem. This onestage method contributes to helping each other in a reinforcement fashion. In the next section, an alternating optimization strategy is used to solve the problem Eq. $(51)$.

\subsection{Optimization Strategy}

It is challenging to obtain an optimized solution for each variable in Eq. (51) since they are coupled together. To solve the challenging problem, an alternating optimization strategy is used to update the variables iteratively. To to be more specific, the variables updated in the last step can be considered as constants in the current step. In this chapter, we have variables $\left\{A^{v}\right\}_{v=1}^{m},\left\{F^{v}\right\}_{v=1}^{m}, H,\left\{w_{v}\right\}_{v=1}^{m}$, $S$, and $Q$ to be optimized. We present the updated rules in the following sections.

\subsubsection{Fix $\left\{F^{v}\right\}_{v=1}^{m}, H,\left\{w_{v}\right\}_{v=1}^{m}, S$, and $Q$, Update $\left\{A^{v}\right\}_{v=1}^{m}$}

When we fix $\left\{F^{v}\right\}_{v=1}^{m}, H,\left\{w_{v}\right\}_{v=1}^{m}, S$, and $Q$, the last four terms of Eq. (51) are constants. Thus, updating $\left\{A^{v}\right\}_{v=1}^{m}$ is to solve the following problem

$$
\begin{aligned}
& \min _{\left\{A^{v}\right\}_{v=1}^{m}} \sum_{v=1}^{m} \sum_{i, j=1}^{n_{v}}\left\|u_{i}^{v}-u_{j}^{v}\right\|_{2}^{2} a_{i j}^{v}+\alpha \sum_{v=1}^{m}\left\|A^{v}\right\|_{F}^{2}+2 \lambda_{1} \sum_{v=1}^{m} \operatorname{Tr}\left(F^{v T} L_{a}^{v} F^{v}\right) \\
& \text { s.t. } \forall v, a_{i i}^{v}=0, a_{i j}^{v} \geq 0, \mathbf{1}^{T} \mathrm{a}_{i}^{v}=1 .
\end{aligned}
$$

It can be seen that each $A^{v}$ can be updated independently. Then, we can update $A^{v}$ one by one using the following equation

$$
\begin{aligned}
& \min _{A^{v}} \sum_{i, j=1}^{n_{v}}\left\|\mathrm{u}_{i}^{v}-\mathrm{u}_{j}^{v}\right\|_{2}^{2} a_{i j}^{v}+\alpha \sum_{i, j=1}^{n_{v}}\left(a_{i j}^{v}\right)^{2}+2 \lambda_{1} \operatorname{Tr}\left(F^{v T} L_{a}^{v} F^{v}\right) \\
& \text { s.t. } a_{i i}^{v}=0, a_{i j}^{v} \geq 0, \mathbf{1}^{T} \mathrm{a}_{i}^{v}=1 .
\end{aligned}
$$

where the last term in Eq. (53) can be transformed as follows

$$
2 \lambda_{1} \operatorname{Tr}\left(F^{v T} L_{a}^{v} F^{v}\right)=\sum_{i, j=1}^{n_{v}} \lambda_{1}\left\|\mathrm{f}_{i}^{v}-\mathrm{f}_{j}^{v}\right\|_{2}^{2} a_{i j}^{v}
$$


Denote $e_{i j}^{u v}=\left\|\mathrm{u}_{i}^{v}-\mathrm{u}_{j}^{v}\right\|_{2}^{2}$ and $e_{i j}^{f v}=\left\|\mathrm{f}_{i}-\mathrm{f}_{j}\right\|_{2}^{2}$. We can also know that Eq. (53) is independently among different vectors $\mathrm{a}_{i}^{v}$ s. Hence, $\mathrm{a}_{i}^{v}$ can be obtained by solving

$$
\begin{aligned}
& \min _{\mathrm{a}_{i}^{v}} \sum_{j=1}^{n_{v}}\left[e_{i j}^{u v} a_{i j}^{v}+\alpha\left(a_{i j}^{v}\right)^{2}+\lambda_{1} e_{i j}^{f v} a_{i j}^{v}\right] \Rightarrow \min _{\mathrm{a}_{i}^{v}} \sum_{j=1}^{n_{v}}\left[\left(e_{i j}^{u v}+\lambda_{1} e_{i j}^{f v}\right) a_{i j}^{v}+\alpha\left(a_{i j}^{v}\right)^{2}\right] \\
& \text { s.t. } a_{i i}^{v}=0, a_{i j}^{v} \geq 0, \mathbf{1}^{T} \mathrm{a}_{i}^{v}=1 .
\end{aligned}
$$

Denote $e_{i j}^{v}=e_{i j}^{u v}+\lambda_{1} e_{i j}^{f v}$, then Eq. (55) can be written as follows:

$$
\min _{\mathrm{a}_{i}^{v}}\left\|\mathrm{a}_{i}^{v}+\frac{\mathrm{e}_{i}^{v}}{2 \alpha}\right\| \text { s.t. } a_{i i}^{v}=0, a_{i j}^{v} \geq 0, \mathbf{1}^{T} \mathrm{a}_{i}^{v}=1 .
$$

where the value of $\alpha$ can be determined according to the number of adaptive neighbors to samples. The "adaptive" means that the $k$ nearest neighbor assignments to each un-missing sample $u_{i}^{v}$ are not fixed and updated in each iteration. From [38], we can know the Eq. (56) has a closed-form solution.

$$
a_{i j}^{v}= \begin{cases}\frac{e_{i, k+1}^{v}-e_{i j}^{v}}{k e_{i, k+1}^{v}-\sum_{t=1}^{k} e_{i t}^{v}} & j \leq k \\ 0 & j>k\end{cases}
$$

3.4.2 Fix $\left\{A^{v}\right\}_{v=1}^{m}, H,\left\{w_{v}\right\}_{v=1}^{m}, S$, and $Q$, Update $\left\{F^{v}\right\}_{v=1}^{m}$

By fixing $\left\{A^{v}\right\}_{v=1}^{m}, H,\left\{w_{v}\right\}_{v=1}^{m}, S$, and $Q$, we update $\left\{F^{v}\right\}_{v=1}^{m}$ according to

$$
\begin{aligned}
& \min _{\left\{F^{v}\right\}_{v=1}^{m}} 2 \lambda_{1} \sum_{v=1}^{m} \operatorname{Tr}\left(F^{v T} L_{a}^{v} F^{v}\right)+\sum_{v=1}^{m} w_{v}\left\|H H^{T}-G^{v T} F^{v} F^{v T} G^{v}\right\|_{F}^{2} \\
& \text { s.t. } F^{v T} F^{v}=\mathbf{I}_{c} .
\end{aligned}
$$

From Eq. (58), the solution for each $F^{v}$ is independent. Thus Eq. (58) can be written as follows

$$
\begin{aligned}
& \min _{F^{v}} 2 \lambda_{1} \operatorname{Tr}\left(F^{v T} L_{a}^{v} F^{v}\right)+w_{v}\left\|H H^{T}-G^{v T} F^{v} F^{v T} G^{v}\right\|_{F}^{2} \\
& \text { s.t. } F^{v T} F^{v}=\mathbf{I}_{c} .
\end{aligned}
$$


According to the properties of the trace operator, the second term without $w_{v}$ can be written below

$$
\begin{aligned}
& \left\|H H^{T}-G^{v T} F^{v} F^{v T} G^{v}\right\|_{F}^{2} \\
& =\operatorname{Tr}\left(\left(H H^{T}-G^{v T} F^{v} F^{v T} G^{v}\right)^{T}\left(H H^{T}-G^{v T} F^{v} F^{v T} G^{v}\right)\right) \\
& =\operatorname{Tr}\left(H H^{T}-2 H H^{T} G^{v T} F^{v} F^{v T} G^{v}+G^{v T} F^{v} F^{v T} G^{v}\right) \\
& =\operatorname{Tr}\left(H H^{T}\right)-2 \operatorname{Tr}\left(H H^{T} G^{v T} F^{v} F^{v T} G^{v}\right)+\operatorname{Tr}\left(G^{v T} F^{v} F^{v T} G^{v}\right) \\
& =\operatorname{Tr}\left(H H^{T}\right)-2 \operatorname{Tr}\left(F^{v T} G^{v} H H^{T} G^{v T} F^{v}\right)+\operatorname{Tr}\left(F^{v T} G^{v} G^{v T} F^{v}\right) \\
& =\operatorname{Tr}\left(H H^{T}\right)-2 \operatorname{Tr}\left(F^{v T} G^{v} H H^{T} G^{v T} F^{v}\right)+\operatorname{Tr}\left(F^{v T} \mathbf{I}_{n_{v}} F^{v}\right)
\end{aligned}
$$

Thus, Eq. (60) is equivalent to the following problem by dropping the unrelated term (i.e., $\left.\operatorname{Tr}\left(H H^{T}\right)\right)$

$$
\begin{aligned}
& \min _{F^{v}} 2 \lambda_{1} \operatorname{Tr}\left(F^{v T} L_{a}^{v} F^{v}\right)-2 w_{v} \operatorname{Tr}\left(F^{v T} G^{v} H H^{T} G^{v T} F^{v}\right)+w_{v} \operatorname{Tr}\left(F^{v T} \mathbf{I}_{n_{v}} F^{v}\right) \\
& \Rightarrow \min _{F^{v}} \operatorname{Tr}\left(F^{v T}\left(2 \lambda_{1} L_{a}^{v}-2 w_{v} G^{v} H H^{T} G^{v T}+w_{v} \mathbf{I}_{n_{v}}\right) F^{v}\right) \\
& \text { s.t. } F^{v T} F^{v}=\mathbf{I}_{c} .
\end{aligned}
$$

Denote $C^{v}=2 \lambda_{1} L_{a}^{v}-2 w_{v} G^{v} H H^{T} G^{v T}+w_{v} \mathbf{I}_{n_{v}}$. In such a way, the optimal solution for $F^{v}$ is the $c$ eigenvectors of $C$ corresponding to the $c$ smallest eigenvalues.

\subsubsection{Fix $\left\{A^{v}\right\}_{v=1}^{m},\left\{F^{v}\right\}_{v=1}^{m},\left\{w_{v}\right\}_{v=1}^{m}, S$, and $Q$, Update $H$}

When $\left\{A^{v}\right\}_{v=1}^{m},\left\{F^{v}\right\}_{v=1}^{m},\left\{w_{v}\right\}_{v=1}^{m}, S$, and $Q$ are fixed, we have

$$
\begin{aligned}
& \min _{H} \sum_{v=1}^{m} w_{v}\left\|H H^{T}-G^{v T} F^{v} F^{v T} G^{v}\right\|_{F}^{2}+\sum_{i, j=1}^{n}\left\|\mathrm{~h}_{i}-\mathrm{h}_{j}\right\|_{2}^{2} s_{i j} \\
& \text { s.t. } H^{T} H=\mathbf{I}_{c} .
\end{aligned}
$$

The second term can be transformed into the following equation

$$
\sum_{i, j=1}^{n}\left\|\mathrm{~h}_{i}-\mathrm{h}_{j}\right\|_{2}^{2} s_{i j}=2 \operatorname{Tr}\left(H^{T} L_{s} H\right)
$$


where $L_{s} \in \mathbb{R}^{n \times n}$ is the Laplacian matrix of the consensus graph $S$. Thus, according to Eq. (60) and (63), we drop the unrelated terms and have

$$
\begin{aligned}
& \min _{H} \sum_{v=1}^{m} w_{v}\left(\operatorname{Tr}\left(H H^{T}\right)-2 \operatorname{Tr}\left(F^{v T} G^{v} H H^{T} G^{v T} F^{v}\right)\right)+2 \operatorname{Tr}\left(H^{T} L_{s} H^{v}\right) \\
& \Rightarrow \min _{H} \sum_{v=1}^{m} w_{v}\left(\operatorname{Tr}\left(H^{T} \mathbf{I}_{n} H\right)-2 \operatorname{Tr}\left(H^{T} G^{v T} F^{v} F^{v T} G^{v} H\right)\right)+2 \operatorname{Tr}\left(H^{T} L_{s} H^{v}\right) \\
& \Rightarrow \min _{H} \operatorname{Tr}\left(H^{T}\left(\sum_{v=1}^{m} w_{v}\left(\mathbf{I}_{n}-2 G^{v T} F^{v} F^{v T} G^{v}\right)+2 L_{s}\right) H\right) \\
& \text { s.t. } H^{T} H=\mathbf{I}_{c} .
\end{aligned}
$$

Denote $M=\sum_{v=1}^{m} w_{v}\left(\mathbf{I}_{n}-2 G^{v T} F^{v} F^{v T} G^{v}\right)+2 L_{s}$. The optimal solution for $H$ is formed by the $c$ eigenvectors of $L_{s}$, which correspond to the $c$ smallest eigenvalues.

\subsubsection{Fix $\left\{A^{v}\right\}_{v=1}^{m},\left\{F^{v}\right\}_{v=1}^{m}, H, S$, and $Q$, Update $\left\{w_{v}\right\}_{v=1}^{m}$}

By fixing $\left\{A^{v}\right\}_{v=1}^{m},\left\{F^{v}\right\}_{v=1}^{m}, H, S$, and $Q$, updating $\left\{w_{v}\right\}_{v=1}^{m}$ is to solve the following problem

$$
\min _{\left\{w_{v}\right\}_{v=1}^{m}} \sum_{v=1}^{m} w_{v}\left\|H H^{T}-G^{v T} F^{v} F^{v T} G^{v}\right\|_{F}^{2}
$$

It can be seen that each $w_{v}$ is updated independently. Eq. (26) can be written as

$$
\min _{w_{v}} w_{v}\left\|H H^{T}-G^{v T} F^{v} F^{v T} G^{v}\right\|_{F}^{2}
$$

From [38], we can know $w_{v}$ will be updated according to the following equation

$$
w_{v}=\frac{1}{2 \sqrt{\left\|H H^{T}-G^{v T} F^{v} F^{v T} G^{v}\right\|_{F}^{2}}}
$$

3.4.5 Fix $\left\{A^{v}\right\}_{v=1}^{m},\left\{F^{v}\right\}_{v=1}^{m}, H,\left\{w_{v}\right\}_{v=1}^{m}$, and $Q$, Update $S$

When we fix $\left\{A^{v}\right\}_{v=1}^{m},\left\{F^{v}\right\}_{v=1}^{m}, H,\left\{w_{v}\right\}_{v=1}^{m}$, and $Q$, we have

$$
\begin{aligned}
& \min _{S} \sum_{i, j=1}^{n}\left\|\mathrm{~h}_{i}-\mathrm{h}_{j}\right\|_{2}^{2} s_{i j}+\beta\|S\|_{F}^{2}+2 \lambda_{2} \operatorname{Tr}\left(Q^{T} L_{s} Q\right) \\
& \text { s.t. } s_{i i}=0, s_{i j} \geq 0, \mathbf{1}^{T} \mathrm{~s}_{i}=1 .
\end{aligned}
$$


Similar to Eq. (54), the last term can be transformed as follows

$$
2 \lambda_{2} \operatorname{Tr}\left(Q^{T} L_{s} Q\right)=\sum_{i, j=1}^{n} \lambda_{2}\left\|\mathrm{q}_{i}-\mathrm{q}_{j}\right\|_{2}^{2} s_{i j}
$$

We denote $d_{i j}^{h}=\left\|\mathrm{h}_{i}-\mathrm{h}_{j}\right\|_{2}^{2}$ and $d_{i j}^{s}=\left\|\mathrm{q}_{i}-\mathrm{q}_{j}\right\|_{2}^{2}$. Hence, Eq. (68) can be written

$$
\begin{aligned}
& \min _{S} \sum_{i, j=1}^{n}\left(d_{i j}^{h} s_{i j}+\beta\left(s_{i j}\right)^{2}+\lambda_{2} d_{i j}^{s} s_{i j}\right) \\
& \text { s.t. } s_{i i}=0, s_{i j} \geq 0, \mathbf{1}^{T} \mathrm{~s}_{i}=1 .
\end{aligned}
$$

Updating $\mathrm{s}_{i}$ is also independent for Eq. (70). Therefore, it can be known that

$$
\begin{aligned}
& \min _{\mathrm{s}_{i}} \sum_{j=1}^{n}\left(\left(d_{i j}^{h}+\lambda_{2} d_{i j}^{s}\right) s_{i j}+\beta\left(s_{i j}\right)^{2}\right) \\
& \text { s.t. } s_{i i}=0, \quad s_{i j} \geq 0, \mathbf{1}^{T} \mathrm{~s}_{i}=1 .
\end{aligned}
$$

We denote $d_{i j}=d_{i j}^{h}+\lambda_{2} d_{i j}^{s}$. Eq. (71) can be written

$$
\min _{\mathrm{s}_{i}}\left\|\mathrm{~s}_{i}+\frac{d_{i}}{2 \beta}\right\| \text { s.t. } s_{i i}=0, s_{i j} \geq 0, \mathbf{1}^{T} \mathrm{~s}_{i}=1 .
$$

where the value of $\beta$ can be determined according to the number of adaptive neighbors. Similar to Eq. (57), the Eq. (72) has a closed-form solution shown in below

$$
s_{i j}= \begin{cases}\frac{d_{i, k+1}-d_{i j}}{k d_{i, k+1}-\sum_{t=1}^{k} d_{i t}} & j \leq k \\ 0 & j>k\end{cases}
$$

\subsubsection{Fix $\left\{A^{v}\right\}_{v=1}^{m},\left\{F^{v}\right\}_{v=1}^{m}, H,\left\{w_{v}\right\}_{v=1}^{m}$, and $S$, Update $Q$}

By fixing $\left\{A^{v}\right\}_{v=1}^{m},\left\{F^{v}\right\}_{v=1}^{m}, H,\left\{w_{v}\right\}_{v=1}^{m}$, and $S$, we can have

$$
\min _{Q} \operatorname{Tr}\left(Q^{T} L_{s} Q\right) \text { s.t. } Q^{T} Q=\mathbf{I}_{c} .
$$

It is well known that the optimal solution $Q$ is the $c$ eigenvectors of $L_{s}$, which correspond to the $c$ smallest eigenvalues.

So far, all the variables can be updated. As we can see, one variable (e.g., $F^{1}, \ldots, F^{v}$ in Eq. (61)) is updated by the other variables (e.g., $\left\{A^{v}, w_{v}\right\}_{v=1}^{m}$, and H). The procedures of our proposed JPG method are shown in Algorithm 2. 
Algorithm 2 JPG Optimization Method

Input: Incomplete multi-view data set of $m$ views $X^{1}, \ldots, X^{m}$ with $X^{v} \in \mathbb{R}^{d_{v} \times n}$, the number of clusters $c$, the number of anchor neighbors $k$, initial parameters $\alpha, \beta, \lambda_{1}, \lambda_{2}$.

Output: The final cluster labels $Y$

1: Initialize the authentic data set $U^{1}, \ldots, U^{m}$ with $U^{v} \in \mathbb{R}^{d_{v} \times n_{v}}$, and index matrices $G^{1}, \ldots, G^{m}$ with $G^{v} \in \mathbb{R}^{n_{v} \times n}$.

2: Initialize the authentic similarity matrices $A^{1}, \ldots, A^{m}$ by Eq. (56), where let $e_{i j}^{v}=e_{i j}^{u v}, \forall v$.

3: Initialize the weight for each view $w_{v}=1 / m, \forall v$.

4: Initialize the local partition matrices $F^{1}, \ldots, F^{m}$ by Eq. (61), where let $C^{v}=$ $2 \lambda_{1} L_{a}^{v}+w_{v} \mathbf{I}_{n_{v}}, \forall v$.

5: Initialize the unified partition $H$ by Eq. (64), where let $M=\sum_{v=1}^{m} w_{v}\left(\mathbf{I}_{n}-\right.$ $\left.2 G^{v T} F^{v} F^{v T} G^{v}\right)$

6: Initialize the consensus graph $S$ by Eq. (72), where let $d_{i j}=d_{i j}^{h}$.

7: Initialize $Q$ by solving Eq. (74).

8: repeat

9: $\quad$ Update $A^{1}, \ldots, A^{m}$ by Eq. (56).

10: $\quad$ Update $F^{1}, \ldots, F^{m}$ by Eq. (61).

11: $\quad$ Update $H$ by Eq. (64).

12: $\quad$ Update $w_{1}, \ldots, w_{m}$ by Eq. (66).

13: $\quad$ Update $S$ by Eq. (72).

14: $\quad$ Update $Q$ by Eq. (74).

15: until Converge

The final clusters $Y$ are the exact $c$ components in the consensus graph matrix $S$.

\subsubsection{Determine the Values of Parameters Adaptively}

In our proposed JPG method, we have four parameters to be determined: $\alpha, \beta, \lambda_{1}$, and $\lambda_{2}$. In simulations, $\alpha \in \mathbb{R}^{n_{v} \times m}$ is actually a parameter matrix; $\beta \in \mathbb{R}^{n \times 1}$ is actually a parameter vector; $\lambda_{1} \in \mathbb{R}^{m \times 1}$ is a parameter vector; $\lambda_{2}$ is a real number. All of them can be adaptively determined in the iterations.

$\alpha$ and $\beta$ are regularization parameters to control the connection sparsity between data samples. Let us take $\alpha=\left[\alpha^{1}, \ldots, \alpha^{m}\right]$ as an example. In Eq. (56), the value of $\alpha_{i}^{v} \in \alpha^{v}$ determines the number of nearest neighbors connected to data sample $\mathrm{a}_{i}^{v}$. If $\alpha_{i}^{v}=0$, the number of nearest neighbors is one. If $\alpha_{i}^{v}$ is infinite, the number of nearest neighbors will be $\left(n_{v}-1\right)$. Since there are $k$ nearest neighbors 
assigned to each sample. Thus, for each $\mathrm{u}_{i}^{v}$, the Lagrangian Function can be

$$
\mathcal{L}\left(\mathrm{a}_{i}^{v}, \phi, \varphi\right)=\frac{1}{2}\left\|\mathrm{a}_{i}^{v}+\frac{\mathrm{e}_{i}^{v}}{2 \alpha_{i}^{v}}\right\|_{2}^{2}-\phi\left(\mathbf{1}^{T} \mathrm{a}_{i}^{v}-1\right)-\varphi^{T} \mathrm{a}_{i}^{v}
$$

where $e_{i j}^{v}=e_{i j}^{u v}+\lambda_{1} e_{i j}^{f v} ; \phi$ and $\varphi$ are Lagrangian coefficient scalar and vector, respectively. From the KKT condition, we can know the optimal solution for $a_{i j}^{v}$ is given by

$$
a_{i j}^{v}=\left(-\frac{e_{i j}^{v}}{2 \alpha_{i}^{v}}+\phi\right)_{+}
$$

where $\phi=\frac{2 \alpha_{i}^{v}+\sum_{j=1}^{k} e_{i j}^{v}}{2 k \alpha_{i}^{v}}$. Moreover, that $\mathrm{u}_{i}^{v}$ has $k$ nearest neighbors can be represented by $\left\{a_{i j}^{v}\right\}_{j=1}^{k}>0$ and $a_{i, k+1}^{v}=0$. We make $e_{i 1}^{v}, e_{i 2}^{v}, \ldots, e_{i k}^{v}$ sorted in the ascending order. Then according to Eq. (75) and $\phi$, we have

$$
\frac{k e_{i k}^{v}-\sum_{j=1}^{k} e_{i j}^{v}}{2}<\alpha_{i}^{v} \leq \frac{k e_{i, k+1}^{v}-\sum_{j=1}^{k} e_{i j}^{v}}{2}
$$

To achieve that most $\mathrm{a}_{i}^{v}$ s have exact $k$ non-zero entries, we set $\alpha_{i}^{v}$ to be equal to the right term.

$$
\alpha_{i}^{v}=\frac{k e_{i, k+1}^{v}-\sum_{j=1}^{k} e_{i j}^{v}}{2}
$$

We plug $\alpha_{i}^{v}$ into Eq. (56) and get the final solution for $a_{i j}^{v}$ in Eq. (57). Thus $\alpha_{i}^{v}$ can be set based on the number of adaptive neighbors. We know all the entries of $\alpha$ can be adaptively determined. Similar to $\alpha_{i}^{v}$, the value of $\beta_{i} \in \beta$ can be determined from Eq. (72) as follows

$$
\beta_{i}=\frac{k d_{i, k+1}-\sum_{j=1}^{k} d_{i j}}{2}
$$

We plug $\beta_{i}$ into Eq. (72) and get the final solution for $s_{i j}$ in Eq. (73).

Moreover, $\lambda_{1}$ and $\lambda_{2}$ are balancing parameters. When they are large enough, the optimal solutions to Eq. (51) will make both $\sum_{v=m}^{c} \sum_{t=1}^{c} \sigma_{t}\left(L_{a}^{v}\right)=0$ and $\sum_{t=1}^{c} \sigma_{t}\left(L_{s}\right)=0$ hold. The values of them do not need to be tuned. In each iteration of our JPG method, for $v$-th view, we can increase or decrease the value 
of $\lambda_{1}^{v}$ when the number of connected components in $A^{v}$ is smaller or greater than $c$. The value of $\lambda_{2}$ can also be increased or decreased when the number of connected components in $S$ is smaller or greater than $c$.

In summary, the four parameters can be determined adaptively and do not need to be tuned.

\subsection{Experiments}

In this section, we investigate the performance of our proposed JPG method on three natural incomplete multi-view datasets and four complete multi-view datasets. To compare with the baseline methods, the experiments are conducted on Matlab development environment.

\subsubsection{Experimental Settings}

Datasets. Our proposed JPG approach is experimentally evaluated on seven widely used multi-view benchmark data sets shown in Table 4, where $n$ is the number of total data samples; $m$ is the number of views; $c$ is the number of classes; $n_{v}$ is the number of un-missing data samples in $v$-th view; $d_{v}$ is the number of dimensions of un-missing data samples in $v$-th view. The seven benchmark data sets are 3 Sources $^{1}, \mathrm{BBC}^{2}, \mathrm{BBCSport}^{3}, 100$ Leaves $^{4}, \mathrm{ORL}^{5}$, Caltech101-7 ${ }^{6}$, and Caltech101-207 , in which the first three data sets are natural incomplete and the remaining data sets are complete.

Baselines. The following seven baseline methods are compared with our proposed JPG methods: best single view (BSV) [66], partial multi-view clustering (PVC) [57], multiple incomplete views clustering (MIC) [67], incomplete multi-

\footnotetext{
${ }^{1}$ http://mlg.ucd.ie/datasets/3sources.html

${ }^{2}$ http://mlg.ucd.ie/datasets/segment.html

${ }^{3}$ http://mlg.ucd.ie/datasets/segment.html

${ }^{4}$ https://archive.ics.uci.edu/ml/datasets/One-hundred+plant+ species+leaves+data+set

${ }^{5}$ www.cad.zju.edu.cn/home/dengcai/Data/FaceData.html

${ }^{6}$ http://www.vision.caltech.edu/Image_Datasets/Caltech101

${ }^{7}$ http://www.vision.caltech.edu/Image_Datasets/Caltech101
} 
Table 4. Statistics of experimental data sets

\begin{tabular}{c|ccc|c|c}
\hline Datasets & $\mathrm{n}$ & $\mathrm{m}$ & $\mathrm{c}$ & $n_{v}(v=1, \ldots, m)$ & $d_{v}(v=1, \ldots, m)$ \\
\hline 3Sources & 416 & 3 & 6 & $352 ; 302 ; 294$ & $3560 ; 3631 ; 3068$ \\
BBC & 2225 & 4 & 5 & $1543 ; 1524 ; 1574 ; 1549$ & $4659 ; 4633 ; 4665 ; 4684$ \\
BBCSport & 737 & 2 & 5 & $644 ; 637$ & $3183 ; 3203$ \\
\hline 100Leaves & 1600 & 3 & 100 & $1600 ; 1600 ; 1600$ & $64 ; 64 ; 64$ \\
ORL & 400 & 4 & 40 & $400 ; 400 ; 400 ; 400$ & $256 ; 256 ; 256 ; 256$ \\
Caltech101-7 & 1474 & 6 & 7 & $1474 ; 1474 ; \ldots ; 1474$ & $48 ; 40 ; 254 ; 1984 ; 512 ; 928$ \\
Caltech101-20 & 2386 & 6 & 20 & $2386 ; 2386 ; \ldots ; 2386$ & $48 ; 40 ; 254 ; 1984 ; 512 ; 928$ \\
\hline
\end{tabular}

modal visual data grouping (MIG) [58], doubly aligned incomplete multi-view clustering (DAIMC) [59], incomplete multi-view spectral clustering with adaptive graph learning (IMSC_AGL) [54], and perturbation-oriented incomplete multi-view clustering (PIC) [62].

Parameter Settings. For the comparisons, we downloaded the algorithms from their websites, ran them using their default parameter settings, and reported their best clustering results. All the baselines and our proposed method are implemented in the Matlab development environment. For JPG, we empirically set $k=10$. The initial values of all entries of parameter $\lambda_{1}$ are set to 1 . Their values are adaptively tuned in the optimization procedure of the objective function for each view. To be more specific, in each iteration, we increase one entry $\lambda_{1}^{v}=2 * \lambda_{1}^{v}$ or decrease it $\lambda_{1}^{v}=\lambda_{1}^{v} / 2$ if the connected components of $A^{v}$ is smaller or greater than $c$. This is also true for $\lambda_{2}$. The initial values of $\alpha$ and $\beta$ do not need to be pregiven and can be obtained in each iteration. Three common metrics are utilized to evaluate the clustering performance: the accuracy (ACC), the normalized mutual information (NMI), and the purity (PUR). To randomize the experiments, we run each method for 5 times, and report the means as well as standard deviations for the metrics. 
Incomplete Multiview Data Generation. In our experiments, there are three types of incomplete multi-view data sets.

1) The first incomplete case is that multi-view data are naturally incomplete. As shown in Table 4, we have three natural incomplete multi-view datasets: 3Sources, BBC, and BBCSport. We directly perform our proposed JPG method and all the baselines on these three datasets in the first case.

2) The second incomplete case is that few samples have complete views. Following the experimental settings in the baselines PVC, IMG, and PIC, we set different partial example ratios (PERs) for 100Leaves, ORL, Caltech101-7, and Caltech101-20 datasets. PER varies from 0.1 to 0.9 with an interval of 0.2 . That is to say, we randomly select $10 \%, 30 \%, 50 \%, 70 \%$, and $90 \%$ of samples and randomly remove certain views with respect to these samples, where the number of removed views is smaller than the total number of views. In this way, each sample is associated with at least one view. We also set $\mathrm{PER}=0$, i.e., the data is complete.

3) The third incomplete case is that all samples have missing views. We use the 100Leaves, 3Sources, and ORL datasets to generate the incomplete multiview data with no paired samples, where about $60 \%$ of samples are randomly removed from each view of the three datasets. For fairness, we repeatedly perform all compared methods five times on these datasets and report their average clustering results.

\subsubsection{Results and Analysis}

Table 5 shows the experimental results of different methods on the incomplete multi-view datasets in the first case. The results include the average metric values 
Table 5. Clustering performance results on three natural incomplete multi-view datasets in the first case

\begin{tabular}{|c|c|c|c|c|c|c|c|c|}
\hline Datasets & BSV & PVC & $\mathrm{MIC}$ & $\mathrm{IMG}$ & DAIMC & IMSC_AGL & PIC & JPG \\
\hline \multicolumn{9}{|c|}{$\mathrm{ACC}$} \\
\hline $\mathrm{BBC}$ & $40.94 \pm 4.20$ & $37.80 \pm 0.97$ & $57.07 \pm 9.74$ & $29.92 \pm 0.01$ & $51.34 \pm 7.44$ & $90.44 \pm 0.04$ & $87.03 \pm 0.05$ & $92.02 \pm 0.84$ \\
\hline BBCSport & $40.99 \pm 0.81$ & $44.33 \pm 1.46$ & $58.66 \pm 9.39$ & $37.86 \pm 0.07$ & $75.16 \pm 8.82$ & $76.89 \pm 0.07$ & $76.02 \pm 5.28$ & $80.19 \pm 4.00$ \\
\hline 3Sources & $5.30 \pm 0.85$ & $1.77 \pm 0.25$ & $38.94 \pm 5.58$ & $2.00 \pm 0.12$ & $48.80 \pm 7.27$ & $66.06 \pm 0.14$ & $73.50 \pm 1.45$ & $76.05 \pm 0.07$ \\
\hline $\mathrm{BBC}$ & $25.99 \pm 2.33$ & $14.72 \pm 0.11$ & $39.19 \pm 6.54$ & $6.24 \pm 0.01$ & $37.74 \pm 7.23$ & $78.54 \pm 0.01$ & $70.12 \pm 0.02$ & $81.62 \pm 1.86$ \\
\hline BBCSport & $26.60 \pm 0.48$ & $13.77 \pm 1.67$ & $46.26 \pm 6.74$ & $7.55 \pm 0.03$ & $57.80 \pm 9.53$ & $73.98 \pm 1.00$ & $75.12 \pm 2.05$ & $79.58 \pm 4.17$ \\
\hline \multicolumn{9}{|c|}{ PUR } \\
\hline
\end{tabular}

and the standard deviations, which are denoted as ave \pm std. We highlight the best results in bold. From the table, we have the following observations.

- Our proposed JPG method acquires better performance than the baselines. In terms of ACC, NMI, and PUR, JPG achieves the best on each data set. The shown results demonstrate that our JPG method is a promising incomplete multi-view clustering approach.

- For the baselines, MIC, DAIMC, IMSC_AGL, and PIC methods outperform BSV, PVC, and IMG. The results show the superiority of multi-view clustering methods (MIC, DAIMC, IMSC_AGL, and PIC) compared with the single view clustering method (BSV) and the two-view clustering methods (PVC and IMG). The reason is that multi-view clustering methods take the differences of different views into account and can better exploit the complementary information of multiple views. It can also be noticed that PVC and IMG do not always perform better than BSV. All of them are inferior to our proposed JPG method.

Figure 9, 10 and 11 show the experimental results of different methods with different PER settings on the incomplete multi-view datasets in the second case. 


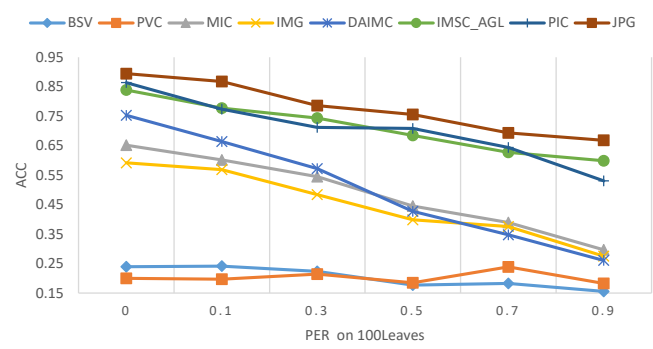

(a)

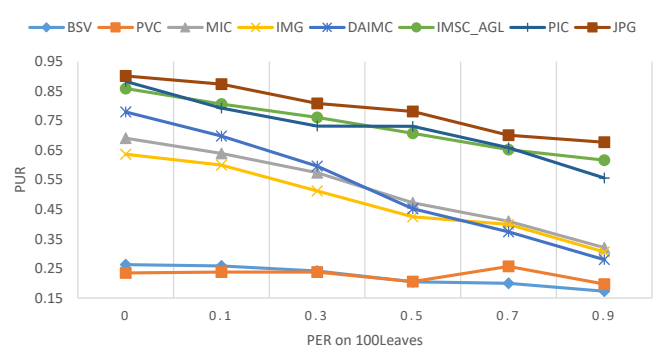

(c)

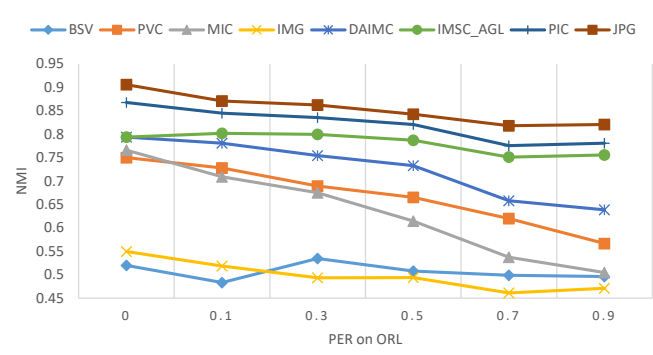

(e)

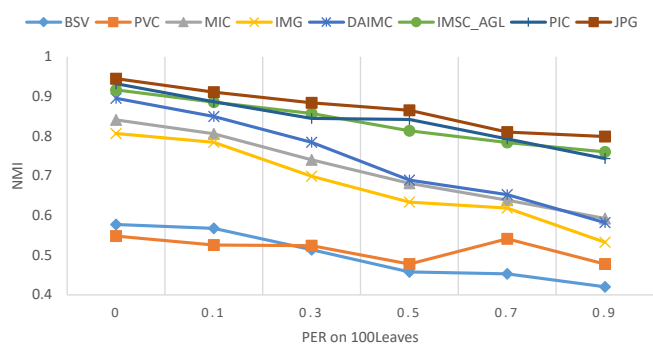

(b)

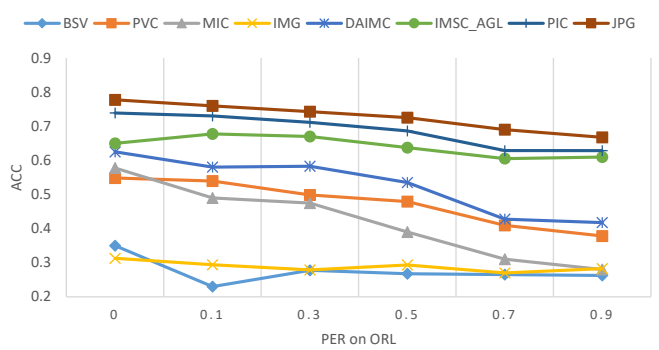

(d)

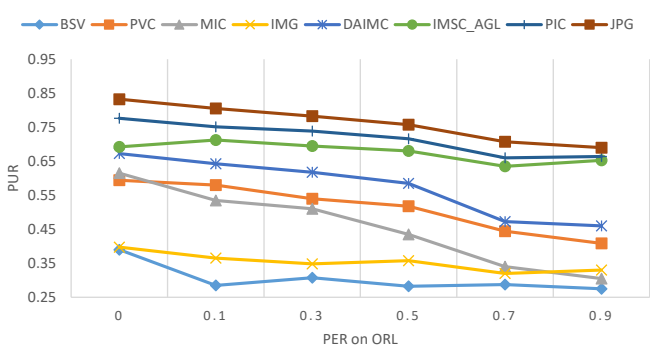

(f)

Figure 9. Clustering performance results with different PER settings in the second case: (a)-(c) on 100Leaves dataset; (d)-(f) on ORL dataset.

For the results of all the methods with different PER settings on the 100Leaves dataset, the metric values of ACC, NMI, and PUR are respectively shown in Figure 9 (a)-(c) and the average metric values of them are shown in Figure 11 (a). For example, the average ACC value for BSV in Figure 11 (a) is computed by averaging all the ACC values in different PERs of Figure 9 (a). Similarly, for that on the ORL dataset, the metric values of ACC, NMI, and PUR are respectively shown in Figure 9 (d)-(f) and the average metric values of them are shown in Figure 11 (b). Figure 10 (a)-(c) show the three metric values on Caltech101-7 dataset and Figure 11 (c) shows the average metric values. Figure 10 (d)-(f) show the three 


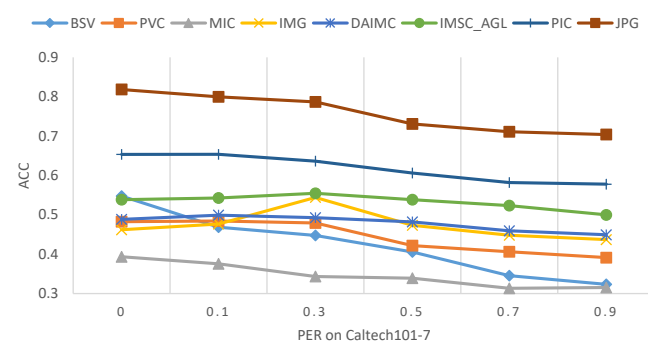

(a)

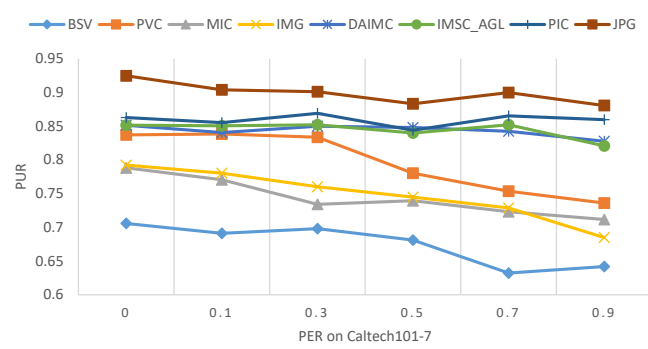

(c)

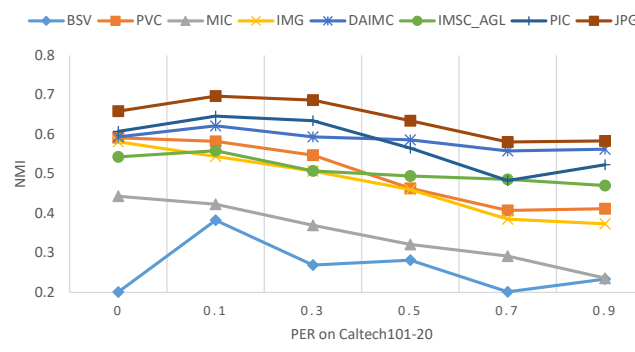

(e)

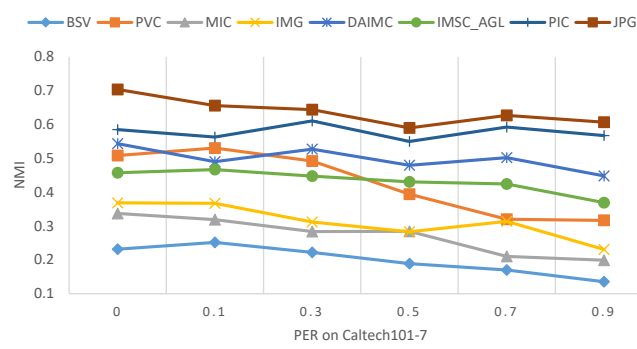

(b)

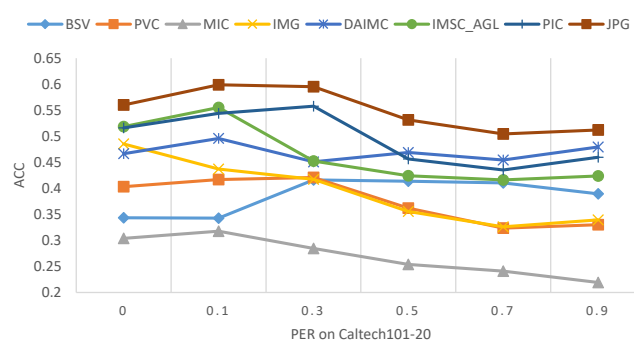

(d)

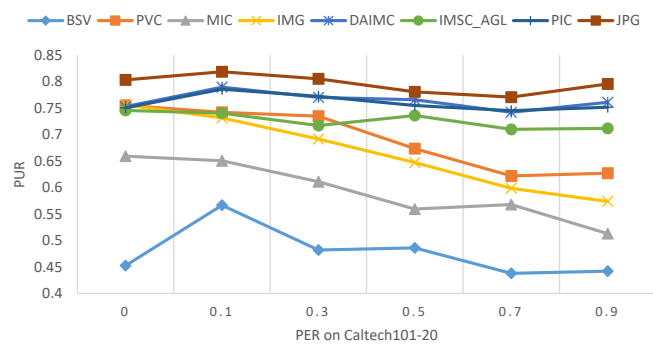

(f)

Figure 10. Clustering performance results with different PER settings in the second case: (a)-(c) on Caltech101-7 dataset; (d)-(f) on Caltech101-20 dataset.

metric values on Caltech101-20 dataset and Figure 11 (d) shows the average metric values. From these figures, we have the following observations.

- Our proposed JPG method is markedly better than all the baselines. In terms of the three metrics and the six PER settings, JPG performs the best on each data set. As the PER increases, the clustering performance of all the approaches drops. It can also be noticed that JPG can address both complete and incomplete multi-view clustering problems.

- In most cases, BSV performs the worst compared with the other approaches. 


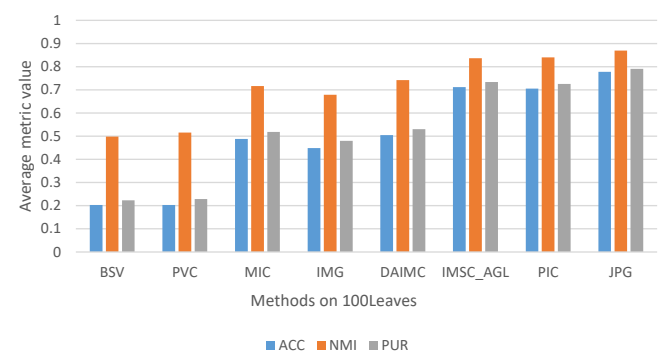

(a)

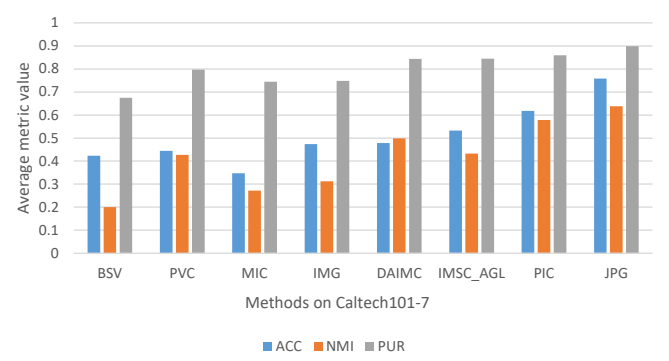

(c)

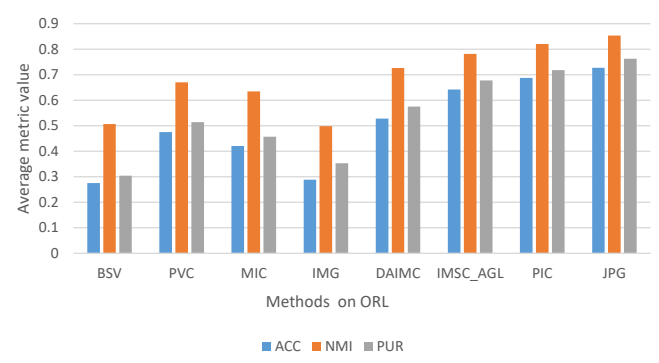

(b)

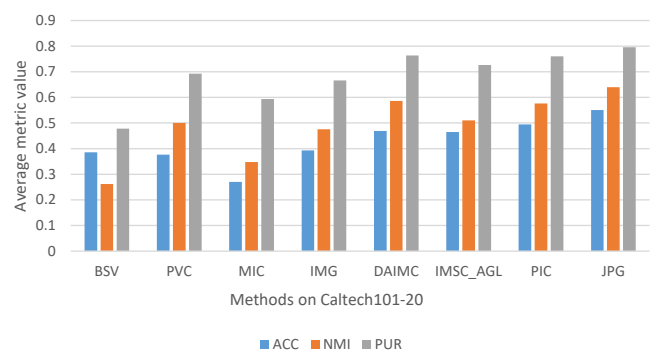

(d)

Figure 11. Average clustering performance results with different PER settings in the second case: on (a) 100Leaves dataset; (b) on ORL dataset; (c) on Caltech101-7 dataset; (d) on Caltech101-20 dataset.

Table 6. Clustering performance results on three incomplete multi-view datasets in the third case

\begin{tabular}{ccccc}
\hline Datasets & DAIMC & IMSC_AGL & PIC & JPG \\
\hline \multicolumn{5}{c}{ ACC } \\
\hline 100Leaves & $25.97 \pm 0.00$ & $35.44 \pm 0.00$ & $33.35 \pm 0.31$ & $\mathbf{3 9 . 3 8} \pm \mathbf{0 . 1 8}$ \\
3Sources & $35.94 \pm 6.63$ & $51.44 \pm 0.00$ & $41.35 \pm 0.00$ & $\mathbf{6 6 . 5 9} \pm \mathbf{0 . 0 0}$ \\
ORL & $33.38 \pm 0.88$ & $45.25 \pm 0.00$ & $42.67 \pm 0.76$ & $\mathbf{4 9 . 0 0} \pm \mathbf{0 . 5 2}$ \\
\hline \multicolumn{5}{c}{ NMI } \\
\hline 100Leaves & $57.63 \pm 0.00$ & $63.21 \pm 0.00$ & $63.37 \pm 0.15$ & $\mathbf{6 5 . 6 8} \pm \mathbf{0 . 1 2}$ \\
3Sources & $18.65 \pm 6.44$ & $30.72 \pm 0.00$ & $29.50 \pm 0.00$ & $\mathbf{5 2 . 0 0} \pm \mathbf{0 . 0 0}$ \\
ORL & $53.79 \pm 0.01$ & $64.80 \pm 0.00$ & $63.41 \pm 0.63$ & $\mathbf{6 5 . 6 5} \pm \mathbf{0 . 5 9}$ \\
\hline & & PUR & \\
\hline 100Leaves & $27.91 \pm 0.31$ & $38.69 \pm 0.00$ & $35.60 \pm 0.26$ & $\mathbf{4 2 . 0 0} \pm \mathbf{0 . 1 6}$ \\
3Sources & $40.38 \pm 6.46$ & $53.85 \pm 0.00$ & $49.28 \pm 0.00$ & $\mathbf{6 7 . 0 7} \pm \mathbf{0 . 0 0}$ \\
ORL & $35.50 \pm 1.06$ & $47.75 \pm 0.00$ & $45.50 \pm 0.66$ & $\mathbf{5 2 . 5 0} \pm \mathbf{0 . 1 4}$ \\
\hline
\end{tabular}


The missing samples of each view are grouped into the same cluster since they are imputed with the same average values of samples, which makes BSV perform badly especially for the case with a large PER. Thus we can know filling the missing samples with the average values of samples is not a promising option in dealing with the incomplete problem.

- The recent baselines IMSC_AGL and PIC are superior to the other baselines. Both of them try to explore the intrinsic geometric structure of each view and combine them to guide the common representation learning. Their results demonstrate this strategy has the potential to achieve the learning of a more discriminative and compact latent representation for clustering. It is also worth noting that if the learned structure may not be intrinsic especially for the case with a larger number of missing samples. Hence, how to obtain the intrinsic geometric structure of each view is essential. Our proposed JPG method can exploit the joint partition and graph learning to achieve it.

Table 6 shows the experimental results of different methods on three incomplete multi-view datasets in the third case. From the table, it can be known that our proposed JPG method significantly outperforms the other three recent baselines in terms of all three metrics. For example, on the 100Leaves dataset, JPG achieves around $4 \%$ improvement in terms of ACC and PUR compared with the second-best IMSG_AGL. on the 3Sources dataset, the values of ACC, NMI, and PUR are about $15 \%, 12 \%$, and $13 \%$ higher than the second-best IMSG_AGL, respectively. The results strongly demonstrate the effectiveness of our proposed JPG method in dealing with the three cases of incomplete problems for multi-view clustering.

To prove the effectiveness of the employed optimization strategy for the objective function of our proposed JPG method, we show the convergence curves 


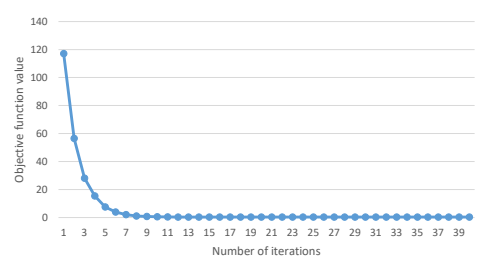

(a)

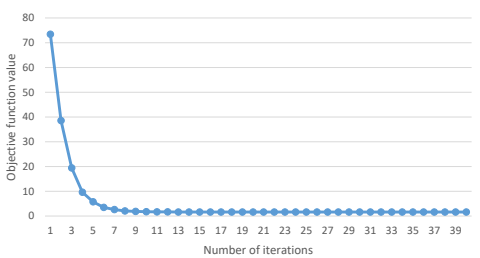

(b)

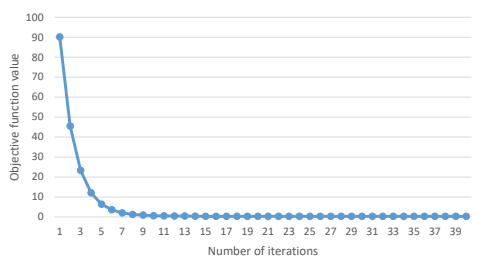

(c)

Figure 12. Convergence curves with 60\% PER settings on (a) 100Leaves data set; (b) 3Sources data set; (c) ORL data set.

of JPG over three incomplete datasets in the third case in Figure 12. For each sub-figure, the $x$-axis denotes the number of iterations and the $y$-axis denotes the objective function value, where the objective function is shown in the above Eq. (51). It can be noticed that JPG converges quickly for all these datasets. To be more specific, it converges within 9 iterations on 100Leaves, 3Sources, and ORL datasets. This indicates that our proposed JPG method has an efficient optimized solution.

\subsection{Chapter Summary}

In this chapter, we proposed a novel joint partition and graph learning method for incomplete multi-view clustering. It jointly learns the local similarity graph and base partition of each view, the unified partition, and a consensus graph in a framework. Moreover, it adaptively determines the importance of each view and directly obtains the final clusters with a low-rank constraint, which is imposed on the consensus graph Laplacian matrix. Finally, the consensus information are uncovered and the clustering structures are learned through an alternating optimization strategy. The experiments on real-world datasets are conducted to demonstrate the effectiveness of our proposed method. 
CHAPTER 4

\section{Unsupervised Domain Adaptation}

\subsection{Background}

Recently, generative adversarial nets (GANs) [68], as popular deep neural net architectures, have been studied heavily. In GANs, two adversarial nets play a minimax game: the generator learns real data distribution to generate new samples to confuse the discriminator, while the discriminator is trained to distinguish the real and forged samples for authenticity. It is intuitive that embedding UDA into adversarial learning contributes to making feature representations of the source domain indistinguishable from that of the target domain. Adversarial domain adaptation approaches through an adversarial objective $[69,70]$ have been successfully used to minimize an approximate distance of domain distribution discrepancy. However, these approaches mainly concentrate on the alignment of the global source and target distributions. Complex and diverse structures are not considered underlying the global data distributions [71]. Consequently, it can raise the problems of confusing source with target data and mixing distinguishable structures, leading to poorly matching different distributions and falsely aligning the corresponding distinguishable structures. Therefore, it might not be appropriate to bridge source and target domains without exploring the complex, diverse structures for multicategory scenarios in UDA.

In this chapter, we propose an adversarial domain adaptation via category transfer (ADACT) approach [72]. ADACT allows us to effectively enhance positive transfer by maximally matching domain distributions in multi-category structures, and greatly reduce negative transfer by preventing misalignment of categories underlying different data distributions. ADACT has 4 players: a source feature generator $G_{s}$, a target feature generator $G_{t}$, a label predictor $C_{y}$, and a combi- 


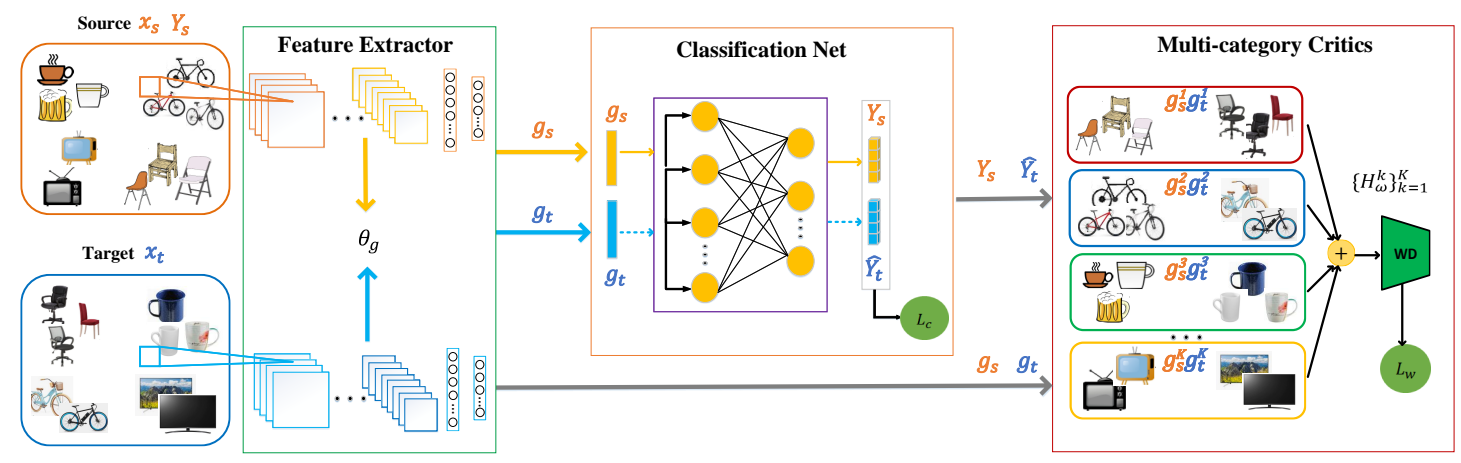

Figure 13. The framework of our proposed ADACT method.

nation of multi-category domain critics $H_{w}$. Firstly, ADACT trains $G_{s}$ and $C_{y}$ with labeled source samples. Then it predicts target labels with target features using the trained $C_{y}$, in which target features are learned by $G_{t}$ initialized with the parameters of trained $G_{s}$. Inspired by recently presented Wasserstein GANs [73], the metric of Wasserstein distance (WD) can conduce to capture categoryinvariant representations between domains. Hence, ADACT category-wisely aligns the corresponding structures using $H_{w}$, which is trained to estimate WDs between source and target feature representations of the same category. Afterward, $G_{t}$ is to be optimized to extract target features that can minimize these category-wise estimated WDs in an adversarial way. Ultimately, we will learn category-invariant feature representations and obtain optimal predicted target labels through iterative adversarial training. Figure 13 shows the overview of ADACT. The effectiveness of ADACT is demonstrated on common DA benchmarks, where ADACT outperforms several state-of-the-art DA methods. Furthermore, visual results of learned representations show the successful alignment of source and target domain distributions. 


\subsection{Related Work}

\subsubsection{Domain Adaptation}

Recent studies are focused on transferring feature representations learned by deep neural networks from a labeled source domain to an unlabeled target domain for UDA $[74,75]$. One effective strategy is to map the features from two different domains into a common latent space, in which the corresponding feature distributions are close [76, 77]. Maximum mean discrepancy (MMD) [78] has been used in several approaches for this purpose. It can measure the divergence between two distribution means in reproducing kernel Hilbert space (RKHS). In deep domain confusion (DDC) [79], MMD is used as a metric of the last fully connected layer in addition to learning domain-invariant and discriminative representations based on the regular classification loss. Deep adaptation network (DAN) [25] utilizes multi-kernel MMD in certain task-specific layers to improve transferability of learned features. Additionally, the correlation alignment (CORAL) [80] uses a linear transformation to perform the alignment of the second-order statistics of two domain distributions. Whereafter, its variant, deep CORAL [81], is proposed to simultaneously match the mean and covariance with a nonlinear transformation.

An adversarial objective is also used to minimize domain discrepancy. Domain adversarial neural network (DANN) [82] is proposed to play a minimax game between feature extractor and domain classifier (a single fully connected layer) to learn domain-invariant features. [69] presents a generalized framework, adversarial discriminative domain adaptation (ADDA), including a domain discriminator, target weight sharing, and an adversarial loss. [83] proposes an adversarial method by weighting the source samples and alleviating the shift of shared classes between domains. Multi-adversarial domain adaptation (MADA) [71] is designed to finely-grained align different data distributions by capturing multi-mode structures using multiple domain discriminators. Similarly, our proposed ADACT can 
also be seen as an adversarial DA approach since it learns category-invariant feature representations in an adversarial manner. Unlike these previous adversarial methods, ADACT iteratively estimates and minimizes WDs between categories in multi-category structures underlying different distributions, which contributes to the alignment of high moments of data distributions.

Another class of divergences between two data distributions is optimal transport (OT) $[84,85]$, where WD induced by OT has been successfully applied to DA due to its generalization [86]. [23] propose WDGRL to learn domain-invariant feature representations by evaluating and minimizing improved WD [87] across domains. Our ADACT differs from these works: i). ADACT trains source and target feature generators separately. ii). ADACT iteratively trains multi-category domain critics such that category-invariant feature representations can be captured through evaluating and minimizing WDs between categories.

Inspired by MADA and WDGRL, we learn category-invariant feature representations with two main differences. Firstly, we have two feature generators: one for the source domain and the other for the target domain. The source feature generator and label predictor are trained by a source encoder with labeled source samples. Secondly, we train the target feature generator by category-wisely minimizing the estimated WDs between corresponding categories, where target labels are predicted by the trained label predictor based on extracted target features at each iteration.

\subsubsection{Discrepancy Metric}

The Wasserstein distance is also known as the Kantorovich-Monge-Rubinstein metric, measuring the distance between probability distributions on a given metric space $M$. It arises from the idea of OT. Let $X \sim \mathbb{P}$ and $Y \sim \mathbb{Q}$. We assume that $X, Y \in \mathbb{R}^{d}$. The Wasserstein distance of order $\sigma$ between two Borel probability 
distribution measures $\mathbb{P}$ and $\mathbb{Q}$ on $M$ is defined as

$$
\begin{aligned}
W_{\sigma}(\mathbb{P}, \mathbb{Q}) & =\left(\inf _{\mu \in \Omega(\mathbb{P}, \mathbb{Q})} \int \eta(x, y)^{\sigma} d \mu(x, y)\right)^{1 / \sigma} \\
& =\inf _{\mu \in \Omega(\mathbb{P}, \mathbb{Q})}\left(\mathbb{E}_{x \sim \mathbb{P}, y \sim \mathbb{Q}} \eta(x, y)^{\sigma}\right)^{1 / \sigma}
\end{aligned}
$$

where $\sigma \geq 1 ; \mathbb{P}, \mathbb{Q} \in\left\{\mathbb{P}: \int \eta(x, y)^{\sigma} d \mathbb{P}(x)<\infty, \forall y \in M\right\} ; \Omega(\mathbb{P}, \mathbb{Q})$ denotes all joint distributions $\mu$ for $(X, Y)$ with marginal distributions $\mathbb{P}$ and $\mathbb{Q} ; \eta$ is a distance and $\eta(x, y)^{\sigma}$ is the corresponding unite cost function; $\mu(x, y)$ can be viewed as a joint probability measure in $\Omega(\mathbb{P}, \mathbb{Q})$, and indicates that how much "mass" would be transported from a random location $x$ to another one $y$ on $M$ such that $\mathbb{P}$ can be transformed into $\mathbb{Q}$. From the above, given an unite cost $\eta(x, y)^{\sigma}$, we can effectively transform $\mathbb{P}$ into $\mathbb{Q}$ at the minimum expected transport $\operatorname{cost} W_{\sigma}(\mathbb{P}, \mathbb{Q})$.

When $M$ is separable and $\sigma=1$, Equation (1) is also called Earth-Mover distance [88]. It can be written as follows

$$
W_{1}(\mathbb{P}, \mathbb{Q})=\sup _{\|f\|_{L} \leq 1} \mathbb{E}_{x \sim \mathbb{P}}[f(x)]-\mathbb{E}_{y \sim \mathbb{Q}}[f(y)]
$$

where $f$ denotes all maps from $\mathbb{R}^{d}$ to $\mathbb{R} ; \eta(x, y) \geq|f(x)-f(y)|$ for all $x, y$; the Lipschitz semi-norm is thus defined as $\|f\|_{L}=\sup |f(x)-f(y)| / \eta(x, y)$. For simplicity, WD is the Wasserstein distance of order 1 in this chapter.

\subsection{Proposed Method}

For the problem of UDA, we are given a labeled source dataset $\left(X_{s}, Y_{s}\right)=$ $\left\{\left(x_{s}^{i}, y_{s}^{i}\right)\right\}_{i=1}^{n_{s}}$ with $n_{s}$ samples from source domain $D^{s}$, and an unlabeled target dataset $X_{t}=\left\{x_{t}^{j}\right\}_{j=1}^{n^{t}}$ with $n_{t}$ samples from target domain $D^{t}$. $D^{s}$ and $D^{t}$ are respectively sampled from joint probability distributions $P$ and $Q$. We assume that $D^{s}$ and $D^{t}$ share the same feature space with total $K$ categories but $P \neq Q$. Our goal is to learn category-invariant and discriminative feature representations using a source feature generator $G_{s}: g_{s}=M_{s}\left(x_{s}\right)$, a label predictor $C_{y}: y=$ $C(g)\left(g \in\left\{g_{s}, g_{t}\right\}\right)$, a target feature generator $G_{t}: g_{t}=M_{t}\left(x_{t}\right)$, and a combination 
of multi-category domain critics: $\left\{H_{w}^{k}\right\}_{k=1}^{K}$ to reduce the distribution discrepancy across domains, such that the target risk $\operatorname{Pr}_{(x, y) \sim q}\left[C\left(M_{t}(x)\right) \neq y\right]$ can be minimized based on multi-category domain adaptation.

\subsubsection{Feature Learning}

Given a sample $x \in \mathbb{R}^{m}$ from either $D^{s}$ or $D^{t}$, the source or target feature generator, $G_{s}$ or $G_{t}$, learns a function: $g_{s}=M_{s}(x)$ or $g_{t}=M_{t}(x)$, which maps the sample from a $m$ - to a $d$-dimensional representation with the parameter $\theta_{s}$ or $\theta_{t}$. The main goal is to learn feature representations of the source and target domains by feature mapping, $M_{s}$ and $M_{t}$, such that the distribution discrepancy across domains can be minimized. Under such circumstances, we can directly apply the source label predictor trained with $g_{s}$ to the target feature representations $g_{t}$. Hence, the supervision information from source data is necessary to be used in category-invariant representation learning process.

Firstly, we train both $G_{s}$ and $C_{y}$ to classify the source samples correctly using the following supervised loss function:

$$
\mathcal{L}_{c}\left(x_{s}, y_{s}\right)=-\mathbb{E}_{\left(x_{s}, y_{s}\right) \sim\left(X_{s}, Y_{s}\right)} \sum_{k=1}^{K} 1_{\left[y_{s}=k\right]} \log C\left(g_{s}\right)
$$

where $1_{\left[y^{s}=k\right]}$ is the indicator function, and $g_{s}=M_{s}\left(x_{s}\right)$. By minimizing softmax

cross entropy to respectively learn the parameters $\theta_{c}, \theta_{s}$ of $C_{y}$ and $G_{s}$, the learning objective function can be attained.

$$
\min _{\theta_{c}, \theta_{s}} \mathcal{L}_{c}
$$

\subsubsection{Category-Invariant Representation Learning}

The label predictor trained by source samples may be highly biased for target samples. To reduce this bias, the challenge of UDA is to match different data distributions of source and target domains. In practical UDA problems, however, 
data distributions also embody complex and diverse structures, which represent category-specific characteristics. Thus, previous UDA approaches that only align global data distributions without considering complex structures and categoryspecific characteristics may raise the problems of negative or under transfer. Negative transfer occurs when we falsely align the categories underlying different distributions, and under transfer occurs when we poorly match distributions of the corresponding categories. In order to improve positive transfer and avoid under transfer, a category-invariant representation learning method is presented to observe complex structures under different data distributions.

In our multi-category adversarial domain adaptation approach, $G_{s}$ is trained well through the pre-training procedure, and then $G_{t}$ is supposed to learn categoryinvariant feature representations with parameter $\theta_{t}$ when $G_{s}$ is fixed. It is noticed that the trained $C_{y}$ using labeled source samples could provide strong signals to observe multi-category structures and capture category-specific characteristics. Thus, we apply the trained $C_{y}$ into the target data $X_{t}$ to initially obtain the corresponding predicted target labels $\widehat{Y}_{t}$.

$$
\widehat{Y}_{t}=C\left(g_{t}\right)
$$

where $g_{t}=M_{t}\left(x_{t}\right)$, and the initial parameter $\theta_{t}$ of $g_{t}$ is set as the trained parameter $\theta_{s}$ of $g_{s}$. To reduce the discrepancy between $D^{s}$ and $D^{t}$ in multi-category structures under data distributions, we use $K$-category domain critics $\left\{H_{w}^{k}\right\}_{k=1}^{K}$, where each is utilized to estimate WD between $D^{s}$ and $D^{t}$ data associated with category $k$.

Given $g_{s}=M_{s}\left(x_{s}\right)$ and $g_{t}=M_{t}\left(x_{t}\right)$, the $k$-th category domain critic $H_{w}^{k}$ learns a function: $g_{w}^{k}$, which maps a $d$-dimensional representation to a real number with parameter $\theta_{w}^{k}$. Afterwards WD between two distributions of category $k, P_{s}^{k}$ and $Q_{t}^{k}$, can be calculated using Eq. 81.

$$
W_{1}\left(P_{s}^{k}, Q_{t}^{k}\right)=\sup _{\left\|g_{w}^{k}\right\| \leq 1} \mathbb{E}_{x \sim P_{s}^{k}}\left[g_{w}^{k}\left(g_{s}^{k}\right)\right]-\mathbb{E}_{x \sim Q_{t}^{k}}\left[g_{w}^{k}\left(\widehat{g_{t}^{k}}\right)\right]
$$


where $g_{s}^{k}$ denotes the source feature representation associated with category $k$ in $Y_{s}$, and $\widehat{g_{t}^{k}}$ represents the target feature representation related to predicted category $k$ in $\widehat{Y}_{t}$. If the parameter of $k$-th category domain critic function $\theta_{w}^{k}$ is 1-Lipschitz, the WD can be estimated by maximizing the $k$-th category domain critic loss $\mathcal{L}_{w}^{k}$ with parameter $\theta_{w}^{k}$

$$
\begin{aligned}
\mathcal{L}_{w}^{k}\left(x_{s}^{k}, \widehat{x_{t}^{k}}\right) & =\frac{1}{n_{s}^{k}} \sum_{x_{s}^{k} \in X_{s}} g_{w}^{k}\left(g_{s}^{k}\right)-\frac{1}{\widehat{n_{t}^{k}}} \sum_{\widehat{x_{t}^{k} \in X_{t}}} g_{w}^{k}\left(\widehat{g_{t}^{k}}\right) \\
& =\frac{1}{n_{s}^{k}} \sum_{x_{s}^{k} \in X_{s}} g_{w}^{k}\left(M_{s}\left(x_{s}^{k}\right)\right)-\frac{1}{\widehat{n_{t}^{k}}} \sum_{\widehat{x_{t}^{k}} \in X_{t}} g_{w}^{k}\left(M_{t}\left(\widehat{x_{t}^{k}}\right)\right)
\end{aligned}
$$

where $k=1,2, \ldots, K ; x_{s}^{k}$ denotes the source samples of category $k ; \widehat{x_{t}^{k}}$ denotes the target samples of predicted category $k ; n_{s}^{k}$ is the number of $k$-th category source samples; $\widehat{n_{t}^{k}}$ is the number of predicted $k$-th category target samples.

However, one problem with WD is that it suffers from training instability. In [73], the weights of domain critic are clipped within a compact space $[-c, c]$ to enforce a Lipschitz constraint. While [87] points out the strategy of weight clipping would induce the issues of gradient vanishing or explode without carefully tuning the threshold $c$. Thus they use gradient penalty to enforce a soft constraint on the gradient norm for random samples. A more reasonable method is to enforce gradient penalty for $k$-category domain critic with parameter $\theta_{w}^{k}$ to learn better representations and enhance training speed.

$$
\mathcal{L}_{g p}^{k}\left(\widehat{h_{k}}\right)=\left(\left\|\nabla_{\widehat{h_{k}}} g_{w}^{k}\left(\widehat{h_{k}}\right)\right\|_{2}-1\right)^{2}
$$

where $\widehat{h_{k}}$ is the point where the gradients are to be penalized by feature representations. The Multi-category domain critics trained with gradient penalty, which are conductive to witness multi-category structures under different distributions. Hence, the category-wise WDs can be estimated by solving the problem

$$
\max _{\theta_{w}^{1}, \ldots, \theta_{w}^{K}}\left\{\mathcal{L}_{w}^{k}+\lambda \mathcal{L}_{g p}^{k}\right\}_{k=1}^{K}
$$


where $\lambda$ is the balancing coefficient.

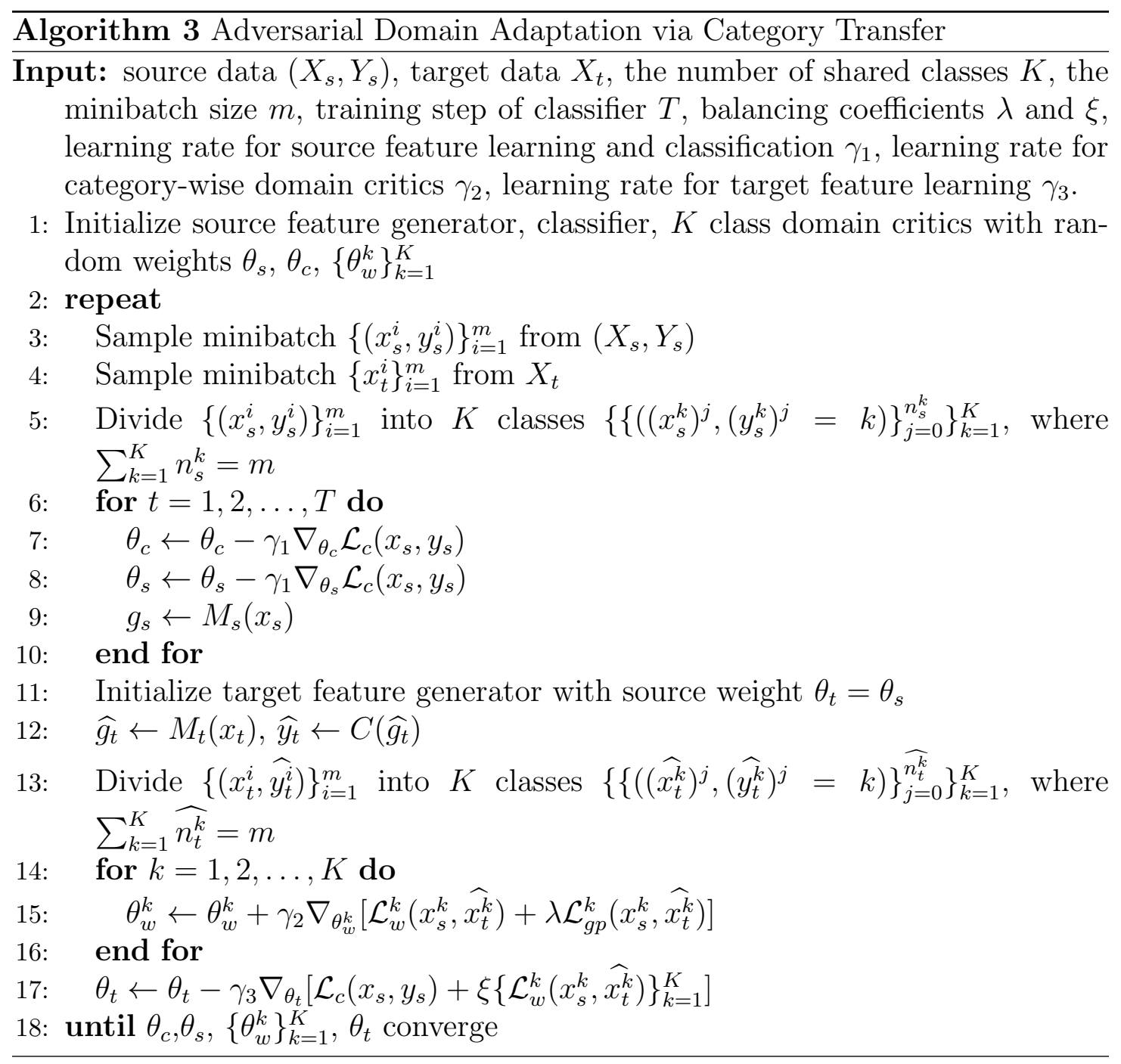

So far, all the variables can be updated. As we can see, one variable (e.g., $F^{1}, \ldots, F^{v}$ in Eq. (61)) is updated by the other variables (e.g., $\left\{A^{v}, w_{v}\right\}_{v=1}^{m}$, and $H)$. The procedures of our proposed JPG method are shown in Algorithm 3.

In view of the continuity and differentiability of WD, we can train all the category domain critics to enable them to be optimized. Then based on the fixed optimal parameters of multi-category domain critics and the minimized estimators of WDs, $G_{t}$ can capture category-invariant feature representations under different distributions. So the target representative features can be learned by addressing 
the minimax problem

$$
\min _{\theta_{t}} \max _{\theta_{w}^{1}, \ldots, \theta_{w}^{K}}\left\{\mathcal{L}_{w}^{k}+\lambda \mathcal{L}_{g p}^{k}\right\}_{k=1}^{K}
$$

where $\theta_{t}$ is the parameter of $G_{t}$, and $\lambda$ is set as 0 during the minimum optimization process. The adversarial objective can be achieved to capture category-invariant features by evaluating and minimizing WDs between categories.

\subsubsection{Overall Objective Function}

The overall objective of adversarial domain adaptation via category transfer $(\mathrm{ADACT})$ is as follows:

$$
\min _{\theta_{c}, \theta_{s}, \theta_{t}}\left\{\mathcal{L}_{c}+\xi \max _{\theta_{w}^{1}, \ldots, \theta_{w}^{K}}\left\{\mathcal{L}_{w}^{k}+\lambda \mathcal{L}_{g p}^{k}\right\}_{k=1}^{K}\right\}
$$

where $\xi$ is the controlling coefficient that balances the discriminative and transferable feature representation learning, and $\lambda$ is set to 0 in the minimum optimization process.

Algorithm 3 shows the detailed training and testing procedures of ADACT. The overall objective can be achieved by the standard back-propagation training approach with a two-step iteration. In ADACT, we first train the label predictor and source feature generator, so that they can be optimized by minimizing the classification loss with labeled source samples. Then we initialize the target feature generator with source weight and use the trained predictor to predict labels of unlabeled target data. After that, the multi-category domain critic networks can be optimized by maximizing the estimators of multi-category WDs via gradient ascent. The target feature generator is finally updated by the combination of the minimized classification loss and the minimized estimated WDs. The learned feature representations can be category-invariant based on multi-category alignment using WDs, and target discriminative due to the parameter $\theta_{t}$ trained by the losses of the classification and multi-category domain critics. 


\subsection{Experiments}

\subsubsection{Experimental Settings}

Datasets. We first evaluate our proposed ADACT in an unsupervised domain adaptation task on three digits datasets: namely, MNIST [89], USPS [90], and Street View House Numbers (SVHN) [91], which are all composed of 10 categories of digits. For the adaptation between MNIST and USPS, two different protocols are tested: one (P1) comprises sampling 2000 MNIST images (abbreviated as M1) and 1800 USPS images (abbreviated as U1), and the other (P2) comprises 70,000 images from the whole MNIST (abbreviated as M2) and 9,298 images from the whole USPS (abbreviated as U2), which are denoted as MNIST and USPS. For the adaptation between MNIST and SVHN, we use M1 and 99,289 SVHN images (abbreviated as S). In all experiments, target labels are withheld. We conduct domain adaptation on the digits datasets in four directions: $\mathrm{M} 1 \rightarrow \mathrm{U} 1, \mathrm{M} 2 \rightarrow \mathrm{U} 2$, $\mathrm{U} 1 \rightarrow \mathrm{M} 1$, and $\mathrm{S} \rightarrow \mathrm{M} 1$. For MNIST $\leftrightarrow$ USPS, USPS images are resized to $28 \times 28$ pixels, which is the size of MNIST images. While for SVHN $\rightarrow$ MNIST, MNIST images are resized to $32 \times 32$ pixels, and SVHN images are converted to grayscale.

We also validate ADACT on the Office-Caltech dataset released by [92], which consists of 10 common classes shared by Office-31 and Caltech-256 datasets. For the adaptation of Office-Caltech, we perform 12 tasks across 4 domains: $\mathrm{A} \rightarrow \mathrm{C}, \mathrm{A}$ $\rightarrow \mathrm{D}, \mathrm{A} \rightarrow \mathrm{W}, \mathrm{C} \rightarrow \mathrm{A}, \mathrm{C} \rightarrow \mathrm{D}, \mathrm{C} \rightarrow \mathrm{W}, \mathrm{D} \rightarrow \mathrm{A}, \mathrm{D} \rightarrow \mathrm{C}, \mathrm{D} \rightarrow \mathrm{W}, \mathrm{W} \rightarrow \mathrm{A}, \mathrm{W} \rightarrow$ $\mathrm{C}$, and $\mathrm{W} \rightarrow \mathrm{D}$, in which the numbers of image samples from Amazon (A), Caltech (C), DSLR (D), and Webcam (W) are 958, 1123, 157, and 295, respectively.

Compared Methods. We compare our proposed ADACT with some of the state-of-the-art unsupervised domain adaptation methods: maximum mean dis-

crepancy metric (MMD) [78], domain adversarial neural network (DANN) [82], adversarial discriminative domain adaptation (ADDA) [69], Wassterstein distance guided representation learning for domain adaptation (WDGRL) [23], adversarial 
feature augmentation for unsupervised domain adaptation (DIFA) [70], maximum classifier discrepancy for unsupervised domain adaptation (MCD) [28], deep correlation alignment (CORAL) [80], unsupervised domain adaptation with distribution matching machines (DMM) [24], and landmarks-based kernelized subspace alignment (LSSA) [93].

Implementation Details. For both digits and Office-Caltech datasets, we use all labeled source data and unlabeled target data, and all categories are trained by Adam optimizer. For each method, the batch size of each domain is set to be 64, and a fixed learning rate is $10^{-4}$. All methods aim at learning domain-invariant and discriminative representations so as to train a classifier that can classify target data correctly. The average classification results for the digits datasets are reported. The best classification results for Office-Caltech are reported.

For the digits dataset, we use a convolutional neural network (CNN) as the basic network architecture of ADACT. The source and target feature generators both have two convolution layers and one fully connected layer, which is defined as conv-pool-conv-pool-fc (with a batch normalization layer before the first convolution layer for SVHN $\rightarrow$ MNIST). The label predictor is defined as fc-softmax. The Office-Caltech dataset uses the representations of DeCAF features [94] as input, which denotes FC7-layer hidden activations with 4,096 dimensions generated by a deep convolutional neural network (AlexNet). A network with two hidden layers of 400 and 100 nodes, relu activation function, and softmax function is designed to further extract features. As before, we fix $\gamma_{1}=\gamma_{2}=\gamma_{3}=10^{-4}$. Transfer cross-validation [95] is also used to choose parameters $\lambda$ and $\xi$. ADACT performs stably when $\lambda=-1$ and $\xi=0.1$.

We follow the suggestions of [23] to integrate MMD, DANN, and CORAL into our own framework. MMD uses 19 RBF kernels, where standard deviation 
parameters range from $10^{-7}$ to $10^{7}$. For DANN experiments, we use a domain classifier with one hidden layer of 100 nodes trained by a gradient reversal layer. CORAL calculates the squared Frobenius norm distance between features from source and target domains using second-order statistics. We add the loss of each of these three methods with a balancing coefficient into classification loss to train the corresponding model. Furthermore, for each approach, we fine-tune its balancing coefficient to achieve the best results. Finally, the balancing coefficients of MMD, DANN, and CORAL are set to $0.3,0.1$, and 0.25 , respectively.

For all compared methods, we follow the suggestions of their corresponding papers to design the network architectures and determine parameter values.

\subsubsection{Results and Analysis}

Table 7. Average accuracy (\%) obtained on digits datasets

\begin{tabular}{ccccccccc}
\hline Datasets & S-only & MMD & DANN & ADDA & WDGRL & DIFA & MCD & ADACT \\
\hline $\mathrm{M} 1 \rightarrow \mathrm{U} 1$ & $89.1 \pm 0.5$ & $92.5 \pm 0.3$ & $91.1 \pm 0.6$ & $89.4 \pm 0.2$ & $94.6 \pm 0.4$ & $92.3 \pm 0.1$ & $94.2 \pm 0.7$ & $\mathbf{9 5 . 7} \pm \mathbf{0 . 2}$ \\
$\mathrm{M} 2 \rightarrow \mathrm{U} 2$ & $88.9 \pm 0.2$ & $96.3 \pm 0.4$ & $95.6 \pm 0.2$ & - & $97.0 \pm 0.3$ & $96.2 \pm 0.2$ & $96.5 \pm 0.3$ & $\mathbf{9 7 . 8} \pm \mathbf{0 . 2}$ \\
$\mathrm{U} 1 \rightarrow \mathrm{M} 1$ & $71.2 \pm 0.3$ & $87.6 \pm 0.5$ & $83.0 \pm 0.3$ & $90.1 \pm 0.8$ & $92.8 \pm 0.5$ & $89.7 \pm 0.5$ & $94.1 \pm 0.3$ & $\mathbf{9 5 . 3} \pm \mathbf{0 . 1}$ \\
$\mathrm{S} \rightarrow \mathrm{M} 1$ & $74.8 \pm 0.4$ & $91.7 \pm 0.1$ & $91.9 \pm 0.2$ & $76.0 \pm 1.8$ & $95.3 \pm 0.4$ & $89.7 \pm 2.0$ & $96.2 \pm 0.4$ & $\mathbf{9 7 . 2} \pm \mathbf{0 . 3}$ \\
\hline
\end{tabular}

Table 7 shows the average classification accuracies (mean \pm std computed over the last iterations) of 7 comparison methods (S-only, MMD, DANN, ADDA, WDGRL, DIFA, and MCD) and our proposed ADACT on 4 transfer tasks of digits datasets. For S-only, we first use labeled source data to train a model (i.e., a source feature generator $G_{s}$ and a label predictor $C_{y}$ ), and then extract target features by the target feature generator $G_{t}$ initialized by the same parameters with $G_{s}$. The target labels are obtained by the trained $C_{y}$ with target features as input. The results of ADDA [69], DIFA [70], and MCD [28] are obtained by the corresponding works in the literature. From the results in Table 1, it can be seen that our proposed ADACT outperforms other current state-of-the-art methods in 
four UDA transfer tasks on digits datasets. Under different frameworks, ADACT performs better than ADDA, WDGRL, DIFA, and MCD since it finely-grained aligns the complex multi-category structures under different data distributions. Under the same framework, ADACT achieves comparable performance to MMD and DANN since the metric of Wasserstein distance with a varying 1-Lipschitz constraint can learn better representations of complex structures.

Table 8. Best accuracy (\%) obtained on Office-Caltech dataset with $\mathrm{DeCAF}_{7}$ features

\begin{tabular}{ccccccccc}
\hline Datasets & S-only & MMD & DANN & CORAL & DMM & WDGRL & LSSA & ADACT \\
\hline $\mathrm{A} \rightarrow \mathrm{C}$ & 84.5 & 89.4 & 87.8 & 86.2 & 83.3 & 87.0 & 82.4 & $\mathbf{9 2 . 7}$ \\
$\mathrm{A} \rightarrow \mathrm{D}$ & 81.1 & 96.4 & 91.2 & 91.2 & 93.0 & 93.7 & 86.4 & $\mathbf{9 6 . 5}$ \\
$\mathrm{A} \rightarrow \mathrm{W}$ & 75.6 & 90.5 & 81.1 & 90.5 & 92.2 & 89.5 & 86.4 & $\mathbf{9 5 . 0}$ \\
\hline $\mathrm{C} \rightarrow \mathrm{A}$ & 92.4 & 93.7 & 92.4 & 93.0 & 92.6 & 93.5 & 91.9 & $\mathbf{9 4 . 3}$ \\
$\mathrm{C} \rightarrow \mathrm{D}$ & 87.7 & 93.0 & 91.2 & 89.5 & 91.7 & $\mathbf{9 4 . 7}$ & 90.4 & 93.0 \\
$\mathrm{C} \rightarrow \mathrm{W}$ & 84.2 & 90.5 & 89.5 & 92.6 & 90.5 & 91.6 & 88.8 & $\mathbf{9 3 . 7}$ \\
\hline $\mathrm{D} \rightarrow \mathrm{A}$ & 84.6 & 93.7 & 87.9 & 85.8 & 93.2 & 91.7 & 86.7 & $\mathbf{9 4 . 9}$ \\
$\mathrm{D} \rightarrow \mathrm{C}$ & 80.5 & 87.0 & 82.9 & 85.4 & 84.3 & $\mathbf{9 0 . 2}$ & 82.5 & 88.7 \\
$\mathrm{D} \rightarrow \mathrm{W}$ & 96.8 & 97.9 & 98.9 & 97.9 & $\mathbf{9 9 . 7}$ & 97.9 & 97.3 & 99.0 \\
\hline $\mathrm{W} \rightarrow \mathrm{A}$ & 79.8 & 93.0 & 82.3 & 88.4 & 92.5 & 93.7 & 88.4 & $\mathbf{9 4 . 2}$ \\
$\mathrm{W} \rightarrow \mathrm{C}$ & 79.7 & 87.8 & 85.6 & 88.6 & 85.8 & 89.4 & 81.6 & $\mathbf{9 0 . 3}$ \\
$\mathrm{W} \rightarrow \mathrm{D}$ & 98.3 & $\mathbf{1 0 0}$ & $\mathbf{1 0 0}$ & $\mathbf{1 0 0}$ & $\mathbf{1 0 0}$ & $\mathbf{1 0 0}$ & 99.4 & $\mathbf{1 0 0}$ \\
\hline $\mathrm{AVG}$ & 85.4 & 92.7 & 87.7 & 91.6 & 92.7 & 88.5 & 90.8 & $\mathbf{9 4 . 4}$ \\
\hline
\end{tabular}

Table 8 shows the best classification accuracies of 7 comparison methods (Sonly, MMD, DANN, CORAL, DMM, WDGRL, LSSA) and our proposed ADACT on 12 transfer tasks of the Office-Caltech dataset using $\mathrm{DeCAF}_{7}$ features. The results of DMM [24], WDGRL [23], and LSSA [24] are obtained by the corresponding works in the literature. It can be observed that our approach performs better than all other comparison approaches in 9 out of 12 UDA tasks, and it scores the second in the remaining 3 tasks. Furthermore, our proposed ADACT significantly outperforms other methods in terms of average classification accuracies. We note that MMD achieves better performance than DANN on both digits and Office-Caltech 
datasets, and MMD outperforms CORAL on the Office-Caltech dataset. However, it still performs worse than ADACT since ADACT can finely-grained align higher moments of data distributions as so to learn category-invariant feature representations.
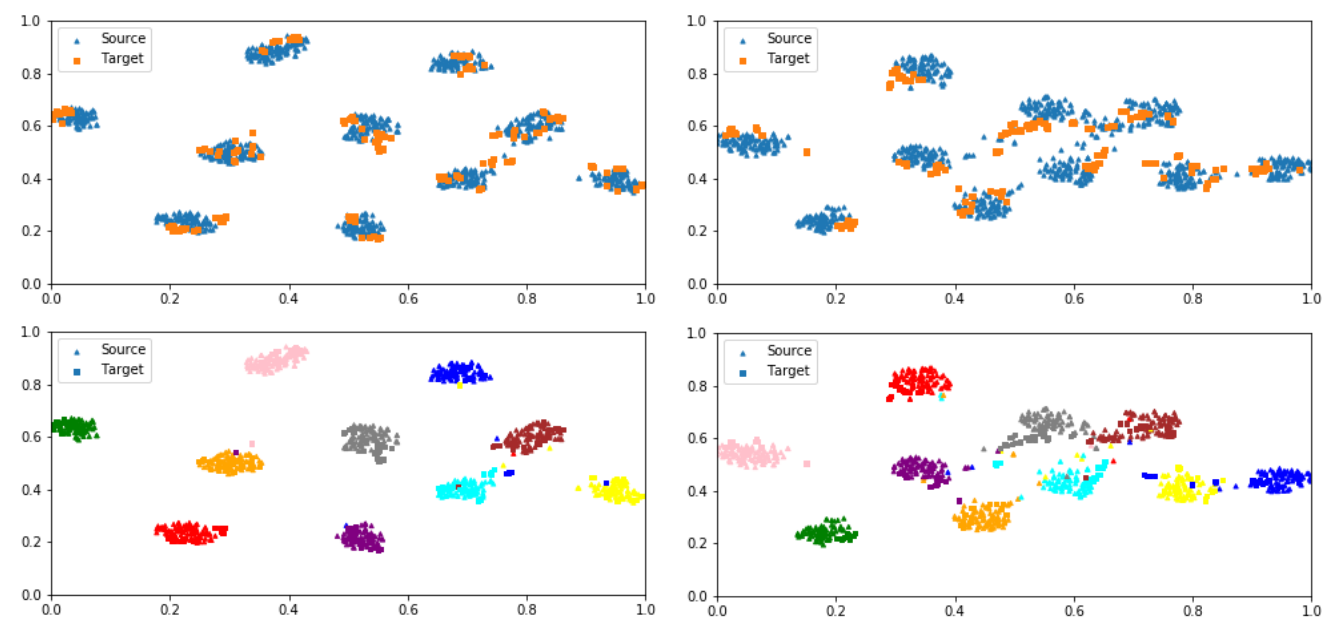

(a) MMD

(b) DANN
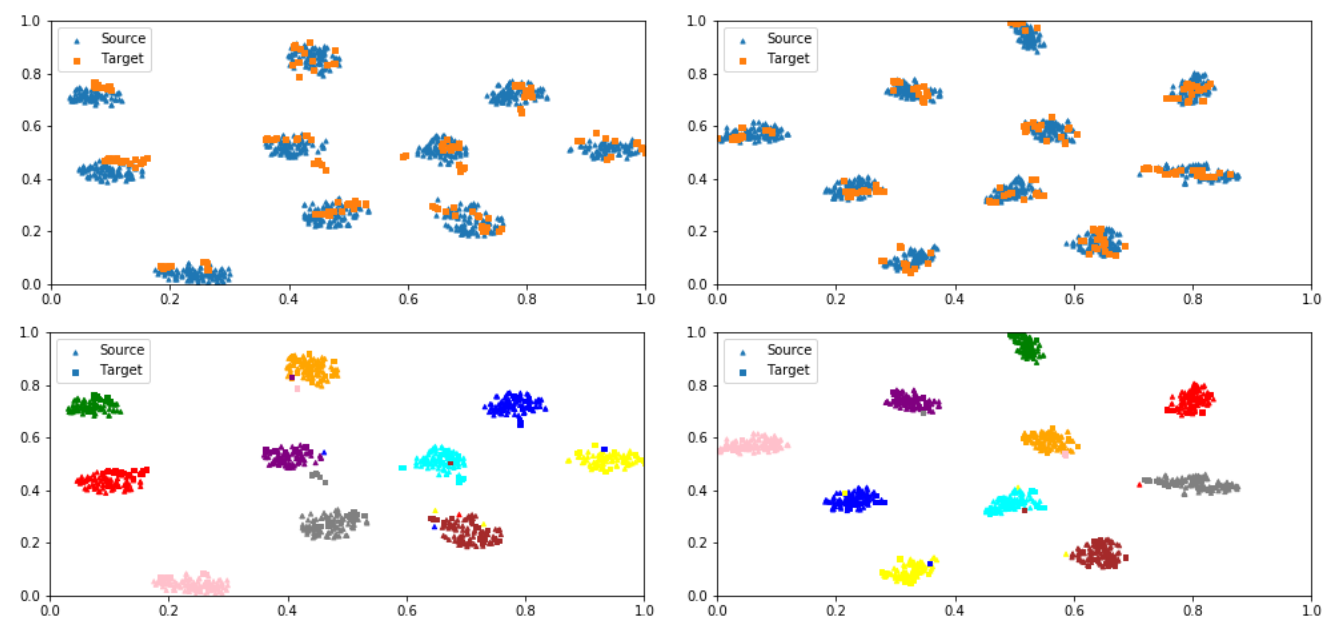

(c) WDGRL

(d) ADACT

Figure 14. The t-SNE visualization of learned features for $\mathrm{A} \rightarrow \mathrm{W}$ task on OfficeCaltech dataset by MMD, DANN, CORAL, WDGRL, and ADACT. The top row shows domain comparisons, where blue triangle and orange square points denote source and target domains, respectively. The bottom row shows category discrimination, where triangle and square points represent source and target domains, and points are colored w.r.t. their classes. 
To further illustrate the effectiveness of our proposed ADACT, as an example, we visualize the learned feature representations in $\mathrm{A} \rightarrow \mathrm{W}$ task on the Office-Caltech dataset, which has 10 shared classes. Figure 14 shows the t-SNE [96] visualization of learned features by the comparison and proposed methods. In the top row, blue triangle points represent source features, and orange square points indicate target features. For a transferable feature mapping in multi-category structures, blue and orange points should be clustered together and indistinguishable. Simultaneously, points with the same color can be easily classified correctly, i.e., clear boundaries exist between classes. The bottom row shows category discrimination. It can be seen that almost all methods learn category-invariant and discriminative feature representations. ADACT performs best since each shared class is clustered better and any two classes have a more clear boundary.

\subsection{Chapter Summary}

In this chapter, we proposed an adversarial domain adaptation via category transfer (ADACT) approach. ADACT can effectively learn category-invariant feature representations in multi-category structures under different data distributions across domains. The data distributions can be finely-grained aligned and the domain discrepancy can be effectively reduced using the metric of Wasserstein distance with gradient penalty. Experimental results on digits and Office-Caltech datasets demonstrate that ADACT outperforms several state-of-the-art unsupervised domain adaptation methods. From feature visualization results, the great learning capability of ADACT is manifested in capturing category-invariant and target-discriminative representations. For future work, we will integrate ADACT into existing unsupervised domain adaptation frameworks, and investigate architectures for tasks in more complex scenarios. 


\section{CHAPTER 5}

\section{Unsupervised Partial Domain Adaptation}

\subsection{Background}

Partial domain adaptation (PDA) assumes the target label space is subsumed into the source label space. In an unsupervised scenario, the target domain only has non-labeled data and the shared label space across domains is unknown. Thus PDA has another technical challenge: how to alleviate the negative transfer caused by the outlier source classes. Recently, there are four related methods including the importance weighted adversarial nets (IWAN) [30], selective adversarial network (SAN) [31], partial adversarial domain adaptation (PADA) [32], and Example Transfer Network (ETN) [33]. They have some successes in addressing the PDA by weighing each sample in the domain-adversarial networks and matching either marginal or conditional distributions to align the source domain as well as the target domain. However, they do not explore the role of each sample, ignore the joint distributions, and do not consider latent structures underlying distributions.

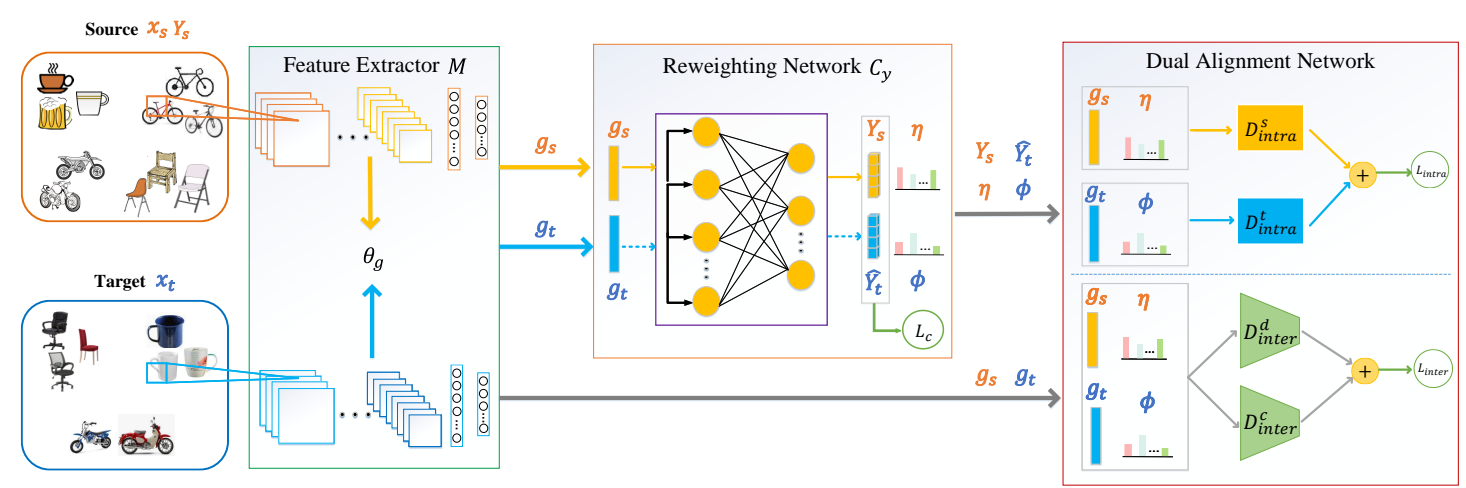

Figure 15. The framework of our proposed DAPDA method.

A dual alignment approach for partial domain adaptation (DAPDA) is presented [97], which improves the previous works [30, 31, 32, 33] by exploring the contribution of each sample and matching joint distributions. Our proposed DAPDA 
method consists of a feature extractor, a reweighting network, and a dual alignment network (shown in Figure 15). The feature extractor $M$ implemented by the Siamese network embeds input samples from source and target domains into latent feature representations. Therein, The Siamese network has two identical sub-networks, where the weights $\theta_{g}$ are shared. This special characteristic of the Siamese network makes it possible to discover the discrepancies between source and target domains. The discrepancies will be minimized during the training process such that the data from the source and target domains can be mapped into the same latent space, i.e., an intermediate domain. With the features extracted by the Siamese network $\left(g_{s}\right.$ and $\left.g_{t}\right)$, we train a reweighting network $C_{y}$ with labeled source data. Then, the trained reweighting network is used to generate "hard" labels $Y_{s}$, class-level weights $\eta$ for source features and "soft" labels $\widehat{Y}_{t}$, instance-level weights $\phi$ for target features. Note that DAPDA improves the reweighting quality over ETN [33] by further learning the contributions of target samples to domain alignment. The dual alignment network aims to match intra-domain and inter-domain distributions based on the Wasserstein distance, which is a metric measuring the difference between distributions. Specifically, intra-domain alignments $\left(D_{\text {intra }}^{s}\right.$ and $D_{\text {intra }}^{t}$ ) would like to minimize the intra-class distances in both source domain and target domain, and inter-domain alignments $\left(D_{\text {inter }}^{d}\right.$ and $\left.D_{\text {inter }}^{c}\right)$ attempt to reduce the discrepancies across domains and that within the same classes from different domains. For our proposed DAPDA method, given source and target domains, we match joint distributions to obtain an intermediate domain, where the learned features from both domains would be class-discriminative and domaininvariant. In such a way, the negative transfer can be alleviated by class-wisely down-weighting source features with non-shared labels; positive transfer can be enhanced by class-wisely up-weighting source features with shared labels as well 
as simultaneously aligning intra-domain and inter-domain distributions; domaininvariant feature representations can be learned through Siamese network in the shared label space.

The main contributions are highlighted as follows:

1) We design a reweighting network to give class-level weights to source features and instance-level weights as well as "soft" labels to target features. The outlier source classes can be down-weighted based on the low class-level weights. The more the target instances are similar to the source domain, the higher instancelevel weights they can have. With each iteration of our proposed method, the errors introduced from wrongly predicted target labels can be reduced.

2) We propose a dual alignment approach for partial domain adaptation to match joint distributions between domains. The proposed method minimizes the intraclass variances in the source domain based on labeled source data, the intra-class variances in the target domain based on weighted target data with "soft" labels, the distances across domains, and the discrepancies within the same class from different domains.

3) We combine both the domain-shared and domain-specific information to learn domain-invariant and class-discriminative feature representations. The proposed model outperforms the existing partial domain adaptation approaches. Good adaptation is achieved in simulations.

The rest of this chapter is organized as follows: Section 5.2 reviews the related work on partial domain adaptation and discrepancy metrics. In Section 5.3, we give the details of the proposed method. Section 5.4 presents the experiments on real-world data sets. In Section 5.5, we provide the conclusion. 


\subsection{Related Work}

Supervised learning has superiority in representation learning. However, the large labeling time and cost hinder its development [98, 6, 40]. Unsupervised learning can discover the hidden patterns without labels $[52,53]$. Reinforcement Learning performs a certain goal by interacting with a dynamic environment [99, 100]. In this chapter, we aim to use unsupervised learning techniques to find the shared space between the source and target domains.

\subsubsection{Partial Domain Adaptation}

Recent studies focus on transferring feature representations learned by deep neural networks from a labeled source domain to an unlabeled target domain. One effective strategy is to map the features from two different domains into a common latent space, in which the corresponding feature distributions are close [76]. Maximum mean discrepancy (MMD) [78] has been used in several approaches for this purpose. It can measure the divergence between two distribution means in reproducing kernel Hilbert space (RKHS). In residual transfer network (RTN) [101], MMD criterion is used to match distributions for a feature adaptation, where the features are fused by the output of multiple layers with the tensor product.

An adversarial objective is also used to minimize domain discrepancy. [69] presents a generalized framework, adversarial discriminative domain adaptation (ADDA), including a domain discriminator, target weight sharing, and an adversarial loss.

Another class of divergences between two data distributions is optimal transport (OT) [84, 85], where Wasserstein distance induced by OT has been successfully applied to domain adaptation due to its generalization $[102,86,73]$. [23] proposes WDGRL to learn domain-invariant feature representations by evaluating

and minimizing improved Wasserstein distance across domains. However, all these 
methods aim to match marginal distributions in identical label space.

For partial domain adaptation problem, four existing methods IWAN [30], SAN [31], PADA [32], and ETN [33]. They address the PDA by weighing each sample in the domain-adversarial networks and matching either marginal or conditional distributions to align the source domain as well as the target domain.

Specifically, IWAN trains the first domain classifier to reweight source domain samples, and matches marginal distributions via a feature extractor as well as the second domain classifier in an adversarial manner. Our proposed method differs from this work: i). For the source domain, the class-level weights are not considered. It may raise the problem of not completely selecting out the outlier source samples, resulting in performance degradation. By contrast, we weight the source domain samples with the average class probabilities overall target samples, which are given by the reweighting network, such that domain-invariant features in the shared label space can be learned. ii). For the target domain, the instancelevel weights are not taken into account. The shared-label source samples may be forcefully aligned to the noise target samples. Our DAPDA method estimates the instance-level weights to target samples reducing the negative effect of noise target samples in a dual alignment network. iii). Conditional distributions are also not considered in IWAN. Instead, we not only finely-grained align source and target domains by category to capture latent structures underlying conditional distributions, but also enhance the intra-class compactness in both domains by minimizing the intra-class variances.

The other three works are proposed by Cao et al. SAN matches conditional distributions across domains by training multiple domain classifiers and downweighting outlier source classes with both class-level and instance-level weights. PADA and ETN focus on matching marginal contributions by training one whole 
domain classifier and down-weighing source outlier classes only with source sample weights, where ETN can automatically obtain source sample weights based on their similarities to the target domain and use the obtained weights in the source classifier as well as domain-adversarial network.

Our proposed method is different from these three works: i). We match joint distributions, starting with the inter-domain alignment and then capturing latent structures by the intra-domain alignment, instead of only matching either marginal or conditional contributions. Specifically, the inter-domain alignment including domain-wise and class-wise alignments contributes to matching both domains in an all-aspect view. ii). We use both class- and instance-level weights and hence are capable of selecting out outlier source samples as well as dealing with imbalanced, noise target data. Since if class-level weights are not applied, outlier source samples may not be picked out completely leading to certain negative transfer. Additionally, if instance-level weights are not applied, the target samples in the negative class (i.e., the class with fewer samples) may not be classified well for the imbalanced issue; the noise target samples will be forcefully aligned to source samples for the noise issue. However, instance-level weights are not considered in PADA and ETN. iii). We assign target samples with "soft" labels and instance-level weights according to maximum class probabilities of Softmax output offered by the reweighting network. Then the distribution discrepancy for each class can be measured by the Wasserstein distance between weighted source and target features. While SAN does not assign labels to target samples and enables all target samples to participate in domain classification in each domain classifier, which makes SAN hardly scalable to large source data and increases computational complexity. 


\subsubsection{Discrepancy Metric}

In this chapter, we use the improved Wasserstein distance as the discrepancy metric of distributions. To enforce the Lipschitz constraint $\|f\|_{L}=\sup \mid f(x)-$ $f(y) \mid / \tau(x, y)$ in Eq. (81), Arjovsky et al. [73] propose to use clipped weights within a compact space $[-c, c]$ after updating gradient. While Gulrajani et al. [87] point out the strategy of weight clipping would induce the issues of gradient vanishing or explode without carefully tuning the threshold $c$. Thus they use gradient penalty to enforce a soft constraint on the gradient norm for random samples $z \sim \mathbb{Z}$.

$$
\begin{aligned}
W_{1}(\mathbb{P}, \mathbb{Q}) & =\sup _{\|f\|_{L} \leq 1} \mathbb{E}_{x \sim \mathbb{P}}[f(x)]-\mathbb{E}_{y \sim \mathbb{Q}}[f(y)] \\
& +\lambda \mathbb{E}_{z \sim \mathbb{Z}}\left[\left(\left\|\nabla_{z} f(z)\right\|_{2}-1\right)^{2}\right]
\end{aligned}
$$

where $z$ is sampled uniformly along straight lines between pairs of points $x$ and $y$; $\lambda$ is a balancing coefficient. The gradients are penalized at $z$. For simplicity, WD is the Wasserstein distance of order 1 in this chapter.

\subsection{Proposed Method}

For the problem of partial domain adaptation in unsupervised scenario [30, $31,32,33]$, we are given a sufficient labeled source dataset $\left(X_{s}, Y_{s}\right)=\left\{\left(x_{s}^{i}, y_{s}^{i}\right)\right\}_{i=1}^{n_{s}}$ with $n_{s}$ samples and known class set $Y_{s}$ from source domain $D^{s}$, and an unlabeled target dataset $\left(X_{t}, Y_{t}\right)=\left\{x_{t}^{j}, y_{t}^{j}\right\}_{j=1}^{n^{t}}$ with $n_{t}$ samples and unknown class set $Y_{t}$ from target domain $D^{t}$. $D^{s}$ and $D^{t}$ share identical feature space but the label space of $D^{t}$ is a subspace of that of $D^{s}$, i.e., $C^{t} \subseteq C^{s}$. $C^{s}$ can be splitted into source domainspecific (outlier) label space and source domain-invariant label space. Additionally, $D^{s}$ and $D^{t}$ are respectively sampled from joint probability distributions $P\left(X_{s}, Y_{s}\right)$ and $Q\left(X_{t}, Y_{t}\right)$, where $P\left(X_{s}, Y_{s}\right) \neq Q\left(X_{t}, Y_{t}\right)$, furthermore, $P\left(X_{s}\right) \neq Q\left(X_{t}\right)$ and $P\left(X_{s} \mid Y_{s}\right) \neq Q\left(X_{t} \mid Y_{t}\right)$. We assume $D^{s}$ have total $K$ known classes $\left|C^{s}\right|=K$, and $\left|C^{t}\right|$ is unknown but $\left|C^{t}\right| \leq\left|C^{s}\right|$. To describe our proposed method better, Table 9 shows the summary of main notations used in this chapter. 
Table 9. Main notations and definitions in the proposed DAPDA method

\begin{tabular}{ll}
\hline Notations & Definitions \\
\hline$D^{s}$ & Source domain \\
$X_{s}$ & Source instances \\
$Y_{s}$ & Source labels (i.e., "hard" labels) \\
$C^{s}$ & Source label space \\
$g_{s}$ & Source features \\
$K$ & Source class number \\
$D^{t}$ & Target Domain \\
$X_{t}$ & Target instances \\
$\widehat{Y}_{t}$ & Predicted target labels (i.e., \\
& "soft" labels) \\
$C^{t}$ & Target label space \\
$g_{t}$ & Target features \\
$M$ & Siamese network \\
$C_{y}$ & Reweighting network \\
$H$ & Dual alignment network \\
$\widehat{q}_{t}$ & Class distribution matrix of tar- \\
& get data over $C^{s}$ \\
$\eta_{c_{k}}$ & Normalized class-level weight for \\
$\phi_{j}$ & source class $c_{k}$ \\
& Instance-level weight for $j$-th tar- \\
\hline
\end{tabular}

Our proposed DAPDA method attempts to use a feature extractor $M$, a reweighting network $C_{y}$, and a dual alignment network $H$ to learn domain-invariant and class-discriminative feature representations as well as reduce joint distribution gaps across domains, such that the target risk $\operatorname{Pr}_{(x, y) \sim Q}\left[C_{y}\left(M\left(x_{t}\right)\right) \neq y_{t}\right]$ in the intermediate domain can be minimized based on the intra- and inter-domain alignments. 


\subsubsection{Feature Extractor}

Siamese network, as the feature extractor, is used to extract domain-invariant features $g$ including two identical sub-networks: one for $D^{s}$ and the other for $D^{t}$. Identical here indicates both sub-networks have the same parameters and weights. In the meantime, parameter updating is mirrored across them. Siamese network has superiority since i) fewer parameters are to be trained which in turn means fewer data are required and less tendency is overfitted; ii) similar model is used to process similar inputs if the inputs are of the same distribution, making feature representations with similar semantics and easier to compare. These special characteristics of Siamese network make it possible to discover the discrepancy between source and target domains. This discrepancy will be minimized during the training process such that the data from the source domain and target domain can be mapped into the same latent space [31, 32]. Through the Siamese network, source features $g_{s}=M\left(x_{s}\right)$ and target features $g_{t}=M\left(x_{t}\right)$ are obtained, where each sample is mapped from a $m$ - to a $d$-dimensional representation with the same parameter $\theta_{g} . \theta_{g}$ can be optimized to enable the Siamese network to learn domaininvariant feature representations by feature mapping $M$, such that positive transfer can be promoted and negative transfer can be alleviated.

\subsubsection{Reweighting Network}

With the extracted source and target features from the Siamese network, reweighting network trained with labeled source feature representations $g_{s}$ can be applied to target feature representations $g_{t}$ to predict their labels. Furthermore, reweighting layer is added to give class-level weights to source features and instance-level weights to target features.

Firstly, we train $C_{y}$ to classify the source samples using the following super- 
vised loss function $[23,31]$ :

$$
\mathcal{L}_{c}\left(x_{s}, y_{s}\right)=\frac{1}{n_{s}} \sum_{i=1}^{n_{s}} L\left(C_{y}\left(g_{s}^{i}\right), y_{s}^{i}\right)
$$

where $L$ is the cross-entropy loss function. By minimizing Softmax cross entropy to respectively learn the parameters $\theta_{c}$ of $C_{y}$, the objective function can be attained.

$$
\min _{\theta_{c}} \mathcal{L}_{c}
$$

Secondly, for source samples at reweighting layer, we up-weight source samples in shared label space and down-weight source samples in non-shared label space. We call the class in shared label space as a shared class and the class in nonshared label space as outlier class. In our proposed DAPDA method, we use $C_{y}$ to determine whether a class is a shared one or not. Specially, we apply $C_{y}$ to the target data $X_{t}$ to obtain the predicted, i.e., "soft" target labels $\widehat{Y}_{t}$.

$$
\widehat{Y}_{t}=C_{y}\left(g_{t}\right)
$$

where $g_{t}=M\left(x_{t}\right)$ with parameter $\theta_{g}$. In the meanwhile, $C_{y}$ also gives a class probability distribution $\widehat{q_{t}^{j}}$ over source label space $C^{s}$ for $j$-th target sample $x_{t}^{j}$. Thus the distribution matrix of target data $\widehat{q_{t}}$ can be obtained as follows:

$$
\widehat{q_{t}}=\left[\begin{array}{c}
\widehat{q_{t}^{1}} \\
\widehat{q_{t}^{2}} \\
\vdots \\
\widehat{q_{t}^{n_{t}}}
\end{array}\right]=\left[\begin{array}{cccc}
\widehat{q_{t, c_{1}}^{1}} & \widehat{q_{t, c_{2}}^{1}} & \cdots & \widehat{q_{t, c_{K}}^{1}} \\
\widehat{q_{t, c_{1}}^{2}} & \widehat{q_{t, c_{2}}^{2}} & \cdots & \widehat{q_{t, c_{K}}^{2}} \\
\vdots & \vdots & \ddots & \vdots \\
\widehat{q_{t, c_{1}}^{n_{t}}} & \widehat{q_{t, c_{2}}^{n_{t}}} & \cdots & \widehat{q_{t, c_{K}}^{n t}}
\end{array}\right]
$$

where $\widehat{q_{t}^{j}}$ represents the probability of assigning $x_{t}^{j}$ to each of $K$ classes and $j=$ $1,2, \ldots, n_{t}$. There should be high probabilities of assigning target samples to the source shared classes. On the contrary, there should be low probabilities of assigning target samples to the source outlier classes. In order to identify the outlier classes, we average the class probabilities $\widehat{q_{t}}$ over all target samples to get 
the source class-level weights. The class with high weight is likely to be shared class while the class with low weight is likely to be outlier class. We then normalize these weights as follows.

$$
\eta_{c_{k}}=\frac{\frac{1}{n_{t}} \sum_{j=1}^{n_{t}} \widehat{q_{t, c_{k}}^{j}}}{\frac{1}{n_{t}} \sum_{c_{k} \in C^{s}} \sum_{j=1}^{n_{t}} \widehat{q_{t, c_{k}}^{j}}}
$$

where $\eta_{c_{k}}$ is normalized source $k$-th source class weight. $\eta=\left\{\eta_{c_{1}}, \eta_{c_{2}}, \ldots, \eta_{c_{K}}\right\}$ represent source class-level weights.

Thirdly, for target samples at reweighting layer, we assign a soft label to each target feature $g_{t}^{j}$ and weight it with its corresponding maximum probability in $\widehat{q_{t}^{j}}$. That is to say, the $j$-th target instance has the soft label $\widehat{y_{t}^{j}} \in C^{s}$ and the weight:

$$
\phi_{j}=\max \left(\widehat{q_{t}^{j}}\right)
$$

The target instance-level weights are denoted as $\phi=\left\{\phi_{j}\right\}_{j=1}^{n_{t}}$. The higher weight of the target feature, the more likely its soft label is to be true. If each target sample is labeled with one specific class (hard label) and weighted by a constant (i.e., 1), it may raise the problem of false alignment, since the reweighting network may make a mistake predicting some samples due to large domain shift. Especially, when some target samples lie in the overlapping area of two classes of distributions, assigning hard labels to these samples and weighting them by a constant would destroy target data structures.

Although the obtained source class-level weights and target instance-level weights can contribute to transferring knowledge from shared source classes and alleviating the negative impact of outlier source classes, these weights highly rely on the probabilities $\widehat{q_{t}}$. Hence, inspired by [103, 31, 33], we employ the entropy minimization principle to refine $C_{y}$. This principle encourages low-density separation between classes such that $C_{y}$ can improve itself to better evaluate target 
unlabeled instances and achieve more accurate probabilities $\widehat{q_{t}}$ with minimal prediction uncertainty. For each target instance, it can be implemented by minimizing the entropy loss:

$$
H\left(C_{y}\left(g_{t}^{j}\right)\right)=-\sum_{k=1}^{K} \widehat{q_{t, c_{k}}^{j}} \log \widehat{q_{t, c_{k}}^{j}} .
$$

Thus, plugging Eq. (98) into Eq. (92), we can have the following loss function to train $C_{y}$ instead of Eq. (92):

$$
\mathcal{L}_{c}\left(x_{s}, y_{s}, x_{t}\right)=\frac{1}{n_{s}} \sum_{i=1}^{n_{s}} L\left(C_{y}\left(g_{s}^{i}\right), y_{s}^{i}\right)+\frac{1}{n_{t}} \sum_{j=1}^{n_{t}} H\left(C_{y}\left(g_{t}^{j}\right)\right)
$$

\subsubsection{Dual Alignment Network}

With the reweighted source and target features from reweighting network, source outlier samples would be down-weighted and source shared samples would be up-weighted. Dual alignment network aims to match joint distributions across domains. The intra-domain alignment attempts to minimize the distance between each instance and its corresponding intra-class centroid for source and target domains, respectively. The inter-domain alignment includes domain-wise alignment and class-wise alignment.

For intra-domain alignment, our goal is to make the learned features in the intermediate domain preserve the intrinsic data structure and the class constraints. That is to say, the features with the same label should be close to the corresponding cluster centroid for both domains. To develop an effective loss term, we first need to determine the centroids for all source and target classes, respectively. Then, for source domain, the loss using the hard labels can be formulated as

$$
\mathcal{L}_{\text {intra }}^{s}=\sum_{k=1}^{K} \frac{1}{n_{s}^{k}} \sum_{y_{s}^{i}=c_{k}}\left\|\eta_{c_{k}} g_{s}^{i}-O_{s}^{k}\right\|^{2}
$$


where

$$
O_{s}^{k}=\frac{1}{n_{s}^{k}} \sum_{y_{s}^{i}=c_{k}} \eta_{c_{k}} g_{s}^{i}
$$

Notably, $O_{s}^{k}$ is the cluster centroid of source class $c_{k}$ calculated by mean value, and $1 / n_{s}^{k}$, as the penalty coefficient, is associated on the distances to balance the effects of different classes. If this coefficient is not involved, the classes over-represented by enough training instances would play a more important role than that underrepresented by only a few. The raised imbalance problem usually results in the degradation of transfer performance on the target domain. Therefore, we attempt to address this problem when the source and target data are imbalanced. For the target domain, the loss using the soft labels can be obtained by

$$
\mathcal{L}_{\text {intra }}^{t}=\sum_{k=1}^{K} \frac{1}{\widehat{n_{t}^{k}}} \sum_{\widehat{y_{t}^{i}=c_{k}}}\left\|\phi_{i} \widehat{g_{t}^{i}}-\widehat{O_{t}^{k}}\right\|^{2}
$$

where

$$
\widehat{O_{t}^{k}}=\frac{1}{\widehat{n_{t}^{k}}} \sum_{\widehat{y_{t}^{i}=c_{k}}} \phi_{i} \widehat{g_{t}^{i}}
$$

For the source domain, we aim to minimize the discrepancies between weighted instances and the centroid in the same class for all classes, which can make the classes discriminative and alleviate the effects of source-specific instances. Similar to the source domain, we embed the instance-level weights such that the target instances with high weights would have more important contributions to enhance the intra-class compactness and the errors introduced from wrongly predicted target labels can be reduced. The intra-domain alignment loss term of both domains can be denoted as

$$
\mathcal{L}_{\text {intra }}=\mathcal{L}_{\text {intra }}^{s}+\mathcal{L}_{\text {intra }}^{t}
$$

Clearly, by minimizing $\mathcal{L}_{\text {intra }}$, the instances with the same label would form compact clusters for both the source and target domains. 
For inter-domain alignment, the domain-wise alignment network maps a $d$ dimensional representation to a real number with parameter $\theta_{d w}$, i.e., $h_{d w}: \mathbb{R}^{d} \rightarrow \mathbb{R}$. Given $g_{s}=M\left(x_{s}\right)$ and $g_{t}=M\left(x_{t}\right)$, the WD with gradient penalty weighted by $\eta$ between two representation distributions, i.e., $P_{g_{s}}$ and $Q_{g_{t}}$ can be calculated using Eq. (91).

$$
\begin{aligned}
W_{1}\left(P_{g_{s}}, Q_{g_{t}}\right) & =\sup _{\left\|h_{d w}\right\| \leq 1} \mathbb{E}_{x \sim P_{g_{s}}}\left[F_{s}\right]-\mathbb{E}_{x \sim Q_{g_{t}}}\left[F_{t}\right] \\
& +\lambda \mathbb{E}_{z_{g} \sim \mathbb{Z}}\left[\left(\left\|\nabla_{z_{g}} F_{z}\right\|_{2}-1\right)^{2}\right]
\end{aligned}
$$

where $F_{s}=h_{d w}\left(\eta g_{s}\right) ; F_{t}=h_{d w}\left(\phi g_{t}\right) ; F_{z}=h_{d w}\left(z_{d}\right)$; source sample $x_{s}^{i}$ with label $c_{k}$ have a corresponding class-level weight $\eta_{c_{k}} ; z_{d}$ are random feature representations sampled along the straight line between pairs of $g_{s}$ and $g_{t}$. If the parameter of the domain-wise alignment network $\theta_{h_{g}}$ is 1-Lipschitz, the WD can be estimated by maximizing the global alignment loss $\mathcal{L}_{d w}$ with parameter $\theta_{d w}$ and balancing coefficient $\lambda_{d w}$

$$
\begin{aligned}
\mathcal{L}_{d w}\left(x_{s}, x_{t}\right) & =\frac{1}{n_{s}} \sum_{x_{s} \in D^{s}} h_{d w}\left(\eta g_{s}\right)-\frac{1}{n_{t}} \sum_{x_{t} \in D^{t}} h_{d w}\left(\phi g_{t}\right) \\
& +\lambda_{d w}\left(\left\|\nabla_{z_{g}} h_{d w}\left(z_{g}\right)\right\|_{2}-1\right)^{2}
\end{aligned}
$$

where $\lambda$ is a balancing coefficient.

However, reducing the discrepancy at the domain level cannot guarantee that the same classes from different domains are pulled close together [31]. To address this problem, it would be necessary to do the class-wise alignment. The classwise alignment network focuses on matching conditional distributions to further explore diverse structures hidden in class characteristics. We have source feature representations with class-level weights and hard labels, as well as target feature representations with instance-level weights and soft labels. If the parameter of the class-wise alignment network $\theta_{c w}$ is 1-Lipschitz, the WD can be estimated by maximizing the class-wise alignment loss $\mathcal{L}_{c w}$ with parameter $\theta_{c w}=\left\{\theta_{c w}^{k}\right\}_{k=1}^{K}$ and 
balancing coefficients $\left\{\lambda_{c w}^{k}\right\}_{k=1}^{K}$.

$$
\begin{aligned}
& \mathcal{L}_{c w}\left(x_{s}, x_{t}\right)=\sum_{k=1}^{K}\left\{\frac{1}{n_{s}^{k}} \sum_{x_{s} \in D_{k}^{s}} h_{c w}^{k}\left(\eta_{c_{k}} g_{s}^{k}\right)-\frac{1}{n_{t}^{k}} \sum_{x_{t} \in \widehat{D_{k}^{t}}} h_{c w}^{k}\left(\widehat{\phi^{k}} \widehat{g_{t}^{k}}\right)\right. \\
& \left.+\lambda_{c w}^{k}\left(\left\|\nabla_{z_{c}^{k}} h_{c w}^{k}\left(z_{c}^{k}\right)\right\|_{2}-1\right)^{2}\right\}
\end{aligned}
$$

where $n_{s}^{k}$ is the number of source instances with hard label $k ; D_{k}^{s}$ indicates all the source instances with hard label $k ; \widehat{n_{t}^{k}}$ is the number of target instances with

soft label $k ; \widehat{D_{k}^{t}}$ represents all the target instances with soft label $k ; \widehat{g_{t}^{k}}$ includes the target features with soft label $k$ and their corresponding instance-level weights $\widehat{\phi^{k}} ; z_{c}^{k}$ are random feature representations sampled along the straight line between pairs of $g_{s}^{k}$ and $\widehat{g_{t}^{k}}$.

We denote the loss term for the inter-domain alignment as $\mathcal{L}_{\text {inter }}=\mathcal{L}_{d w}+\mathcal{L}_{c w}$ with the parameters $\theta_{\text {inter }}=\left\{\theta_{d w}, \theta_{c w}\right\}$. The alignment can be achieved by solving the problem

$$
\max _{\theta_{\text {inter }}} \mathcal{L}_{\text {inter. }}
$$

where balancing coefficients $\lambda_{d w}$ and $\left\{\lambda_{c w}^{k}\right\}_{k=1}^{K}$ should be set to 0 at the end of each iteration of optimizing the maximum. It is because the gradient penalty ought not to guide other learning procedures.

\subsubsection{Overall Objective Function}

The overall objective of our proposed DAPDA method is as follows:

$$
\min _{\theta_{g}, \theta_{c}}\left\{\mathcal{L}_{c}+\alpha \mathcal{L}_{\text {intra }}+\beta \max _{\theta_{\text {inter }}} \mathcal{L}_{\text {inter }}\right\}
$$

where $\alpha$ and $\beta$ are two balancing coefficients. Algorithm 4 shows the detailed training and testing procedures of our proposed method. The overall objective can be achieved by the standard back-propagation training approach with a twostep iteration. We first train the reweighting network, which can be optimized by minimizing the classification loss with labeled source samples. Second, we apply 


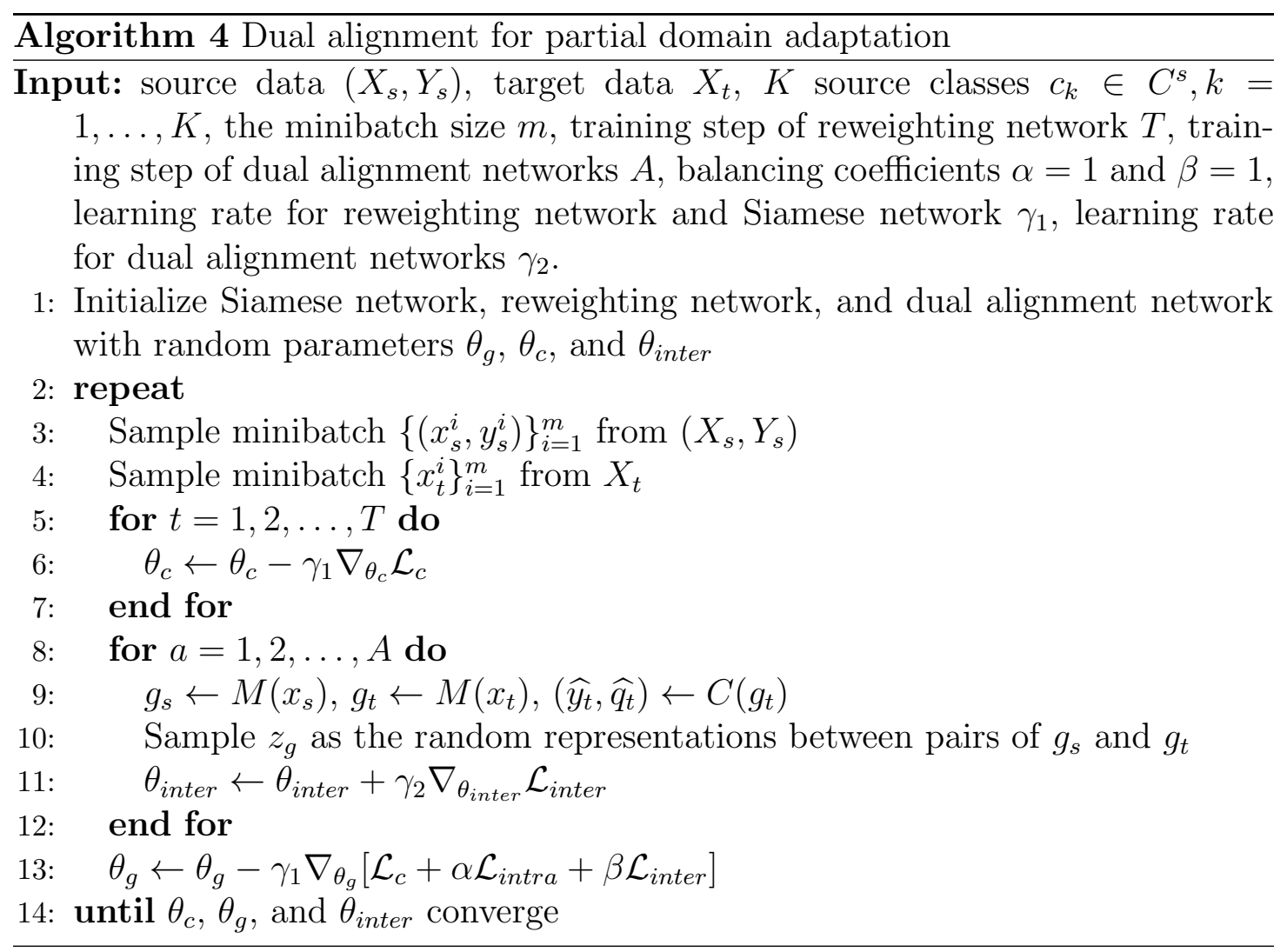

the trained classifier to predict soft labels of target data such that outlier source samples can be down-weighted as well as the soft labels and instance-weights for target samples can be obtained. Then the inter-domain alignment network can be optimized by maximizing the estimators of WDs with gradient penalty via gradient ascent. After this iteration, the balancing coefficients in $\theta_{\text {inter }}$ are set to 0. The Siamese network is finally updated by the combination of the minimized classification loss, the minimized intra-domain alignment loss, and the maximized estimated WDs. The learned feature representations can be domain-invariant and class-discriminative based on dual alignment.

\subsection{Experiments}

\subsubsection{Experimental Settings}

Datasets. Our proposed DAPDA method is evaluated on three widely used real world datasets: Office-31[104], Office-Caltech [92], and Office-Home [105]. 


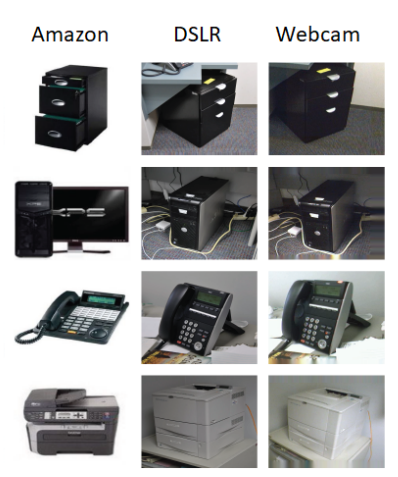

(a) Office-31

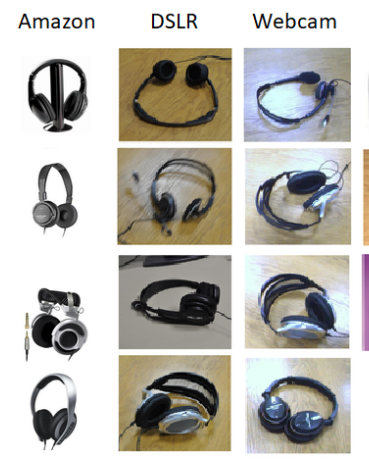

(b) Office-Caltech
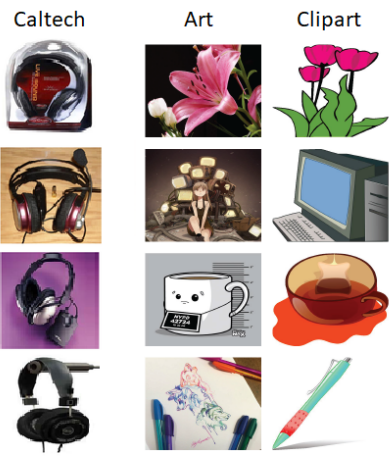

Product RealWorld
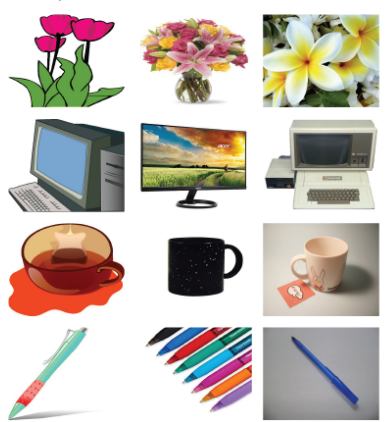

(c) Office-Home

Figure 16. Illustration of several image examples for (a) Office-31 dataset, (b) Office-Caltech dataset, and (c) Office-Home dataset.

Figure 16 shows several image examples for these three datasets.

We first validate DAPDA on the Office-31 dataset, which is a standard benchmark for domain adaptation and composed of 4,652 images and 31 classes. 3 distinct domains are involved: Amazon (A), Webcam (W), and DSLR (D), which include images downloaded from amazon.com, taken with web cameras, and picked up by digital SLR cameras, respectively. We follow the experimental settings of [30, 32], taking one domain with 31 classes as the source domain and another domain with 10 classes (which are shared by Office-31 and Caltech-256) as the target domain to enable adaptation. Hence, 6 transfer tasks across 3 domains are conducted: A31 $\rightarrow$ W10, A31 $\rightarrow$ D10, D31 $\rightarrow$ A10, D31 $\rightarrow$ W10, W31 $\rightarrow$ A10, and $\mathrm{W} 31 \rightarrow \mathrm{D} 10$.

Secondly, we validate DAPDA on the Office-Caltech dataset released by [92], which consists of 10 common classes shared by Office-31 and Caltech-256 datasets. The experimental settings of [30] are also applied, taking one domain with 10 classes as the source domain and another domain with the first 5 classes as the target domain to enable adaptation. For the partial adaptation of Office-Caltech, we perform 12 tasks across 4 domains: $\mathrm{A} 10 \rightarrow \mathrm{C} 5, \mathrm{~A} 10 \rightarrow \mathrm{D} 5, \mathrm{~A} 10 \rightarrow \mathrm{W} 5, \mathrm{C} 10$ $\rightarrow \mathrm{A} 5, \mathrm{C} 10 \rightarrow \mathrm{D} 5, \mathrm{C} 10 \rightarrow \mathrm{W} 5, \mathrm{D} 10 \rightarrow \mathrm{A} 5, \mathrm{D} 10 \rightarrow \mathrm{C} 5, \mathrm{D} 10 \rightarrow \mathrm{W} 5, \mathrm{~W} 10 \rightarrow$ 
$\mathrm{A} 5, \mathrm{~W} 10 \rightarrow \mathrm{C} 5$, and $\mathrm{W} 10 \rightarrow \mathrm{D} 5$, in which the numbers of image samples from Amazon (A), Caltech (C), DSLR (D), and Webcam (W) are 958, 1123, 157, and 295, respectively. Thereinto, A, D, and W domains are from Office-31, and C domain comes from Caltech-256. Additionally, we further conduct experiments on the Office-Caltech dataset in the standard full protocol.

To evaluate it on a large-scale dataset, we design several transfer tasks on the Office-Home dataset, which contains 15,500 images crawled via a few search engines and online image directories. This dataset has 4 domains: Artistic (Ar), Clipart ( $\mathrm{Cl})$, Product $(\mathrm{Pr})$, and Real-World $(\mathrm{Rw})$ images, where each domain includes images from 65 object classes. In each transfer task, one domain with all 65 classes can be considered as the source domain, and another domain with the first 25 classes can be taken as the target domain. Thus, for the partial adaptation of Office-Home, 12 transfer tasks can be performed: Ar65 $\rightarrow$ Pr25, Ar65 $\rightarrow$ Cl25, Ar65 $\rightarrow$ Rw25, Cl65 $\rightarrow$ Pr25, Cl65 $\rightarrow$ Ar25, Cl65 $\rightarrow$ Rw25, Pr65 $\rightarrow$ Ar25, Pr65 $\rightarrow$ $\mathrm{Cl25}$, Pr65 $\rightarrow$ Rw25, Rw65 $\rightarrow$ Pr25, Rw65 $\rightarrow$ Ar25, and Rw65 $\rightarrow$ Cl25.

Benchmark Methods. For partial domain adaptation, we compare our proposed DAPDA with the baseline that finetuning the CNN (e.g., AlexNet [106] and ResNet-50 [107]), and several deep domain adaptation methods: Wassetstein Distance Guided Representation Learning (WDGRL) [23], Adversarial Discriminative Domain Adaptation (ADDA) [69], Reverse Gradient (RevGrad) [76], Residual Transfer Network (RTN) [101], Importance Weighted Adversarial Nets [30], Selective Adversarial Network (SAN) [31], Domain Adversarial Neural Network (DANN) [82], Deep Adaptation Network (DAN) [25], Joint Adaptation Network (JAN) [108], Partial Adversarial Domain Adaptation (PADA) [32], and Example Transfer Network [33].

As we all know, in a dual alignment network, the proposed DAPDA with intra- 
domain alignment only and without the inter-domain alignment would perform worse than vice versa since the distribution gap is not reduced. Moreover, we also would like to explore the effect of the entropy minimization principle for DAPDA. Therefore, to further demonstrate the effectiveness of DAPDA with respect to the inter-domain alignment and the entropy minimization principle, three variants are evaluated by ablation study: i) DAPDA-CW is the variant with the entropy minimization principle and class-wise alignment only, and without domain-wise alignment; ii) DAPDA-DW is the variant with the entropy minimization principle and domain-wise alignment only, and without class-wise alignment; iii) DAPDA-NEN is the variant with class-wise and domain-wise alignments, without the entropy minimization principle.

For full domain adaptation, the compared methods are DANN, DAN, WDGRL, and Distribution Matching Machines (DMM) [24], our proposed method with the metric of Maximum Mean Discrepancy (Ours-MMD) [78], our proposed method with the metric of CORrelation ALignment (Ours-CORAL) [80], in which Ours-MMD indicates the DAPDA with the MMD metric and Ours-CORAL represents the DAPDA with the metric in CORAL (i.e., the second-order statistics).

Implementation Details. Following standard protocols, we use all labeled source data and unlabeled target data for unsupervised domain adaptation. All our models are implemented using TensorFlow and trained by Adam optimizer. For a fair comparison, we fine-tune the AlexNet and ResNet-50, respectively, which are both pre-trained on ImageNet dataset similar to previous domain adaptation approaches [30, 32]. For DAPDA, we fine-tune the two sub-networks of the Siamese network, which is the standard multi-player perceptron network designed with two hidden layers of 500 and 100 nodes for all datasets. The reweighting network is built with one hidden layer of 100 nodes, relu activation function, and softmax output 
function. Our inter-domain alignment network is designed with a hidden layer of 100 nodes. The training steps of reweighting network $T$ and dual alignment networks $A$ are 10 and 5, respectively. The learning rates $\gamma_{1}$ and $\gamma_{2}$ are $10^{-4}$. The gradients are penalized at source, target, and random representations. The balancing coefficients of gradient penalty $\lambda_{d w}$ and $\left\{\lambda_{c w}^{k}\right\}_{k=1}^{K}$ are all set to -10 as suggested in [87].

For each method, the batch size of each domain is set to be 64, and a fixed learning rate is $10^{-4}$. We report the average classification accuracy results of each transfer task over 3 random experiments. The values of hyper-parameters are selected based on their original papers.

\subsubsection{Results and Analysis}

Table 10, 11, 12, and 13 show the average results of the compared and our proposed methods for partial domain adaptation, where the results of IWAN, SAN, PADA, and ETN are copied directly from their corresponding original papers. The best results are marked in bold. To a large extent, our proposed DAPDA method performs better than previous domain adaptation methods, such as AlexNet, WDGRL, ADDA, RevGrad, and RTN. Furthermore, it can be comparable to some state-of-the-art partial domain adaptation approaches, like IWAN, SAN, PADA, and ETN on most datasets.

Table 10 shows the detailed comparison results of these methods using AlexNet as the baseline on Office-31 dataset. Our proposed DAPDA method outperforms all the other methods. Specifically, the average classification accuracy of DAPDA is $94.99 \%$, and DAPDA achieves significant performance enhancements of $7.72 \%$ and $13.45 \%$ compared to the best partial domain adaptation method IWAN as well as the best full domain adaptation method ADDA, respectively. We notice that the partial domain adaptation methods perform better than the full domain 
Table 10. Average accuracy (\%) of partial domain adaptation tasks on the Office31 dataset (AlexNet)

\begin{tabular}{c|cccccc|c}
\hline Methods & $\mathrm{A} 31 \rightarrow \mathrm{D} 10$ & $\mathrm{~A} 31 \rightarrow \mathrm{W} 10$ & $\mathrm{D} 31 \rightarrow \mathrm{A} 10$ & $\mathrm{D} 31 \rightarrow \mathrm{W} 10$ & $\mathrm{~W} 31 \rightarrow \mathrm{A} 10$ & $\mathrm{~W} 31 \rightarrow \mathrm{D} 10$ & $\mathrm{AVG}$ \\
\hline AlexNet & 71.97 & 62.03 & 68.27 & 95.25 & 62.94 & 97.45 & 76.32 \\
WDGRL & 57.14 & 47.66 & 52.06 & 78.08 & 73.87 & 85.66 & 65.75 \\
ADDA & 72.92 & 70.39 & 74.46 & 96.31 & 76.46 & 98.72 & 81.54 \\
RevGrad & 57.32 & 56.59 & 57.62 & 75.59 & 63.15 & 89.17 & 66.64 \\
RTN & 69.43 & 68.14 & 68.27 & 91.53 & 77.35 & 98.09 & 78.80 \\
IWAN & 78.98 & 76.27 & 89.46 & 98.98 & 81.73 & $\mathbf{1 0 0 . 0}$ & 87.57 \\
SAN & 81.28 & 80.02 & 80.58 & 98.64 & 83.09 & $\mathbf{1 0 0 . 0}$ & 87.27 \\
\hline DAPDA-CW & 88.72 & 89.73 & 88.69 & 98.99 & 92.13 & $\mathbf{1 0 0 . 0}$ & 93.04 \\
DAPDA-DW & 85.66 & 88.37 & 87.13 & 97.67 & 90.93 & 99.32 & 91.51 \\
DAPDA-N-EN & 85.82 & 88.84 & 90.81 & 99.43 & 96.32 & $\mathbf{1 0 0 . 0}$ & 93.54 \\
DAPDA & $\mathbf{8 9 . 3 0}$ & $\mathbf{9 2 . 6 1}$ & $\mathbf{9 3 . 1 8}$ & $\mathbf{1 0 0 . 0}$ & $\mathbf{9 6 . 8 3}$ & $\mathbf{1 0 0 . 0}$ & $\mathbf{9 4 . 9 9}$ \\
\hline
\end{tabular}

adaptation methods. The average results of our proposed two variants of DAPDA also are better than most of the compared methods. DAPDA-CW has a slightly better performance than DAPDA-DW since the local characteristics associated with the categories of the Office-31 dataset can greatly contribute to distribution alignment.

Table 11. Average accuracy (\%) of partial domain adaptation tasks on OfficeCaltech dataset (AlexNet)

\begin{tabular}{|c|c|c|c|c|c|c|c|c|c|c|c|c|c|}
\hline Methods & $\begin{array}{r}\mathrm{A} 10 \\
\rightarrow \mathrm{C} 5\end{array}$ & $\begin{array}{l}\text { A10 } \\
\rightarrow \text { D5 }\end{array}$ & $\begin{array}{c}\mathrm{A} 10 \\
\rightarrow \mathrm{W} 5\end{array}$ & $\begin{array}{c}\mathrm{C} 10 \\
\rightarrow \mathrm{A} 5\end{array}$ & $\begin{array}{l}\text { C10 } \\
\rightarrow \text { D5 }\end{array}$ & $\begin{array}{c}\mathrm{C} 10 \\
\rightarrow \mathrm{W} 5\end{array}$ & $\begin{array}{c}\text { D10 } \\
\rightarrow \text { A5 }\end{array}$ & $\begin{array}{l}\text { D10 } \\
\rightarrow \text { C5 }\end{array}$ & $\begin{array}{c}\mathrm{D} 10 \\
\rightarrow \mathrm{W} 5\end{array}$ & $\begin{array}{l}\text { W10 } \\
\rightarrow \mathrm{A} 5\end{array}$ & $\begin{array}{l}\text { W10 } \\
\rightarrow \text { C5 } 5\end{array}$ & $\begin{array}{l}\text { W10 } \\
\rightarrow \text { D5 }\end{array}$ & AVG \\
\hline AlexNet & 85.27 & 85.29 & 76.30 & 93.58 & 91.18 & 83.70 & 89.51 & 80.82 & 98.52 & 87.37 & 74.14 & 100.0 & 87.14 \\
\hline WDGRL & 75.53 & 61.36 & 56.82 & 91.30 & 70.45 & 76.14 & 65.34 & 54.68 & 84.09 & 70.63 & 61.38 & 90.91 & 71.55 \\
\hline RTN & 80.99 & 70.59 & 69.63 & 91.86 & 80.88 & 93.99 & 70.02 & 59.08 & 91.11 & 74.73 & 59.08 & 100.0 & 78.44 \\
\hline IWAN & 89.90 & 88.24 & 87.41 & 94.22 & 98.53 & 97.78 & 94.43 & 91.61 & 98.52 & 95.29 & 90.24 & 100.0 & 93.85 \\
\hline DAPDA-DW & 91.59 & 95.92 & 90.31 & 96.63 & 91.67 & 90.86 & 96.60 & 92.25 & 96.05 & 92.89 & 91.77 & 100.0 & 93.88 \\
\hline DAPDA & 93.05 & 98.83 & 93.46 & 98.66 & 100.0 & 97.93 & 97.02 & 94.62 & 99.10 & 96.62 & 94.35 & 100.0 & 96.97 \\
\hline
\end{tabular}

Similar to Table 10, Table 11 shows the average classification accuracies of several methods using AlexNet as the baseline for 12 partial transfer tasks on the Office-Caltech dataset. DAPDA achieves better performance than most compared methods in 10 out of 12 partial transfer tasks, and it also achieves the secondhighest accuracy in the remaining 2 tasks. It can be noticed that the performance of the baseline is better than the full domain adaptation methods including WDGRL, 
RevGrad, and RTN. This phenomenon indicates the importance of picking out the source outlier classes such that the errors of misalignment can be alleviated.

Table 12. Average accuracy (\%) of partial domain adaptation tasks on Office-31 dataset (ResNet-50)

\begin{tabular}{c|cccccc|c}
\hline Methods & $\mathrm{A} 31 \rightarrow \mathrm{D} 10$ & $\mathrm{~A} 31 \rightarrow \mathrm{W} 10$ & $\mathrm{D} 31 \rightarrow \mathrm{A} 10$ & $\mathrm{D} 31 \rightarrow \mathrm{W} 10$ & $\mathrm{~W} 31 \rightarrow \mathrm{A} 10$ & $\mathrm{~W} 31 \rightarrow \mathrm{D} 10$ & $\mathrm{AVG}$ \\
\hline ResNet & 65.61 & 54.52 & 73.17 & 94.57 & 71.71 & 94.27 & 75.64 \\
WDGRL & 39.03 & 35.24 & 40.61 & 43.29 & 41.06 & 37.30 & 39.42 \\
ADDA & 43.66 & 43.65 & 42.76 & 46.48 & 45.95 & 40.12 & 43.77 \\
DANN & 41.36 & 41.35 & 41.34 & 46.78 & 44.68 & 38.85 & 42.39 \\
DAN & 42.68 & 46.44 & 65.66 & 53.56 & 65.34 & 58.60 & 55.38 \\
RTN & 66.88 & 75.25 & 85.59 & 97.12 & 85.70 & 98.32 & 84.81 \\
JAN & 35.67 & 43.39 & 51.04 & 53.56 & 51.57 & 41.40 & 46.11 \\
PADA & 82.17 & 86.54 & 92.69 & 99.32 & 95.41 & $\mathbf{1 0 0 . 0}$ & 92.69 \\
IWAN & 90.45 & 89.15 & 95.62 & 99.32 & 94.26 & 99.36 & 94.69 \\
SAN & 90.70 & 83.39 & 87.16 & 99.32 & 91.85 & $\mathbf{1 0 0 . 0}$ & 92.07 \\
ETN & $\mathbf{9 5 . 0 3}$ & 94.52 & $\mathbf{9 6 . 2 1}$ & $\mathbf{1 0 0 . 0}$ & 94.64 & $\mathbf{1 0 0 . 0}$ & $\mathbf{9 6 . 7 3}$ \\
\hline DAPDA-N-EN & 84.78 & 90.12 & 93.65 & 99.57 & 97.34 & $\mathbf{1 0 0 . 0}$ & 94.24 \\
DAPDA & 92.15 & $\mathbf{9 5 . 0 6}$ & 95.13 & $\mathbf{1 0 0 . 0}$ & $\mathbf{9 7 . 4 0}$ & $\mathbf{1 0 0 . 0}$ & 96.62 \\
\hline
\end{tabular}

In Table 12, we use the ResNet-50 as the baseline on the Office-31 dataset. DAPDA performs slightly worse than ETN but better than the other methods in the average accuracy. No noticeable degradation is observed compared to the state-of-the-art methods. It can be noticed that the worst-performing method is WDGRL shown in these three tables. That is because WDGRL first aligns the distributions of source and target domains based on WD criterion and then predicts the labels of target samples, which is not suitable for the partial adaptation problem. Starting with alignment without considering the influence of source outliers could easily cause domain confusion and faulty alignment. This also happens to RevGrad based on adversarial nets and RTN based on MMD metric. WDGRL and RevGrad perform worse than standard AlexNet since negative transfer caused by source outliers is not considered. They try to match source and target domains including matching the source outliers and target data to predict labels of target samples in outlier classes as much as possible. ADDA, IWAN, SAN, PADA, and ETN are all adversarial nets-based methods, playing a minimax game between 
feature extractors and domain classifiers by GRL layer. ADDA is an unweighted version of IWAN and thus performs worse than IWAN in detecting source outlier samples. IWAN and PADA focus on selecting out outliers with class-level weights but both of them match different domains without considering latent structures hidden in each class. While SAN uses a separate domain classifier for each class to explore the latent structures ignoring intra-class compactness. ETN performs well by embedding the source sample weights in the source classifier and the domain -adversarial network.

Table 13. Average accuracy (\%) of partial domain adaptation tasks on Office-Home dataset (ResNet-50)

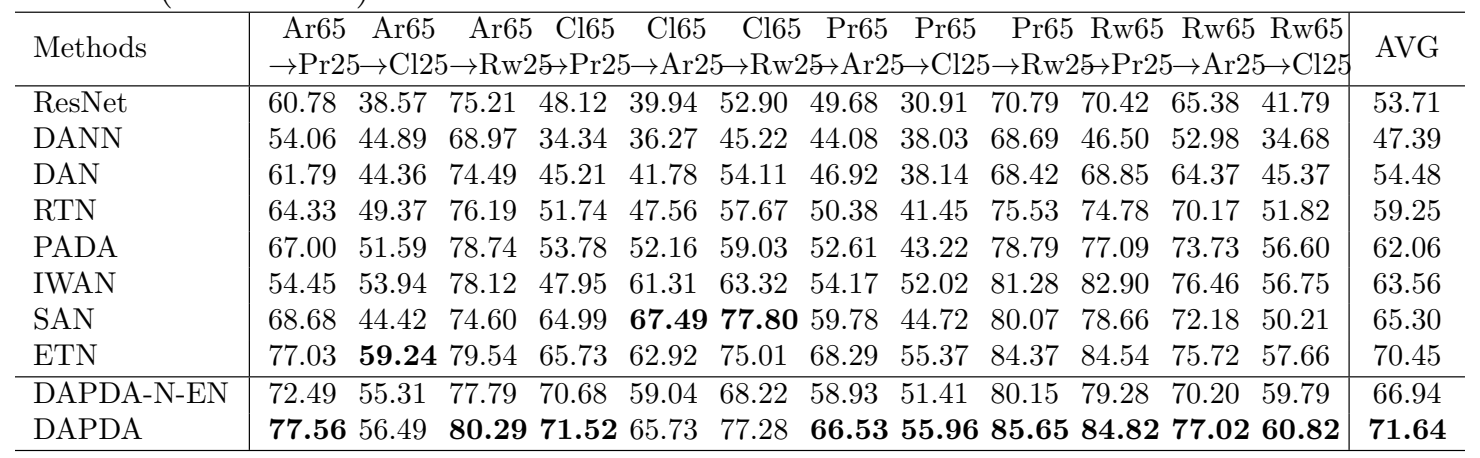

The average classification accuracies of DAPDA and other comparisons on the Office-Home dataset are listed in Table 13. It is worth noting that DAPDA obtains the best performance in 9 out of 12 transfer tasks. We observe that DAPDA enhances the average performance by huge margins not only on Office-Caltech and Office-31 datasets both with small domain gaps but also on Office-Home with large domain gaps. The dual alignment strategy makes DAPDA generalize well on the unlabeled target data.

To further illustrate the effectiveness of our proposed DAPDA, as an example, we visualize the learned feature representations using AlexNet as the baseline in A10 $\rightarrow$ C5 task on the Office-Caltech dataset in Table 10. In Figure 17 and 18, the 10 classes are labeled as 0-9 and 5 shared classes are 0-5 classes. In Figure 17, the 


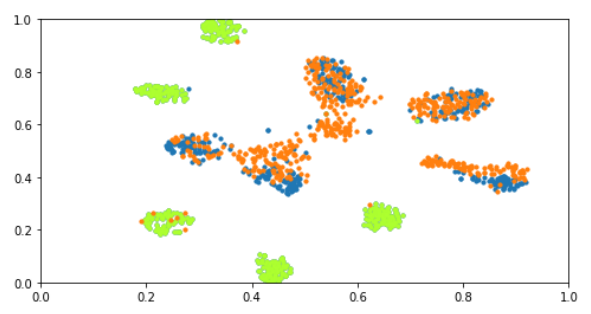

(a) AlexNet

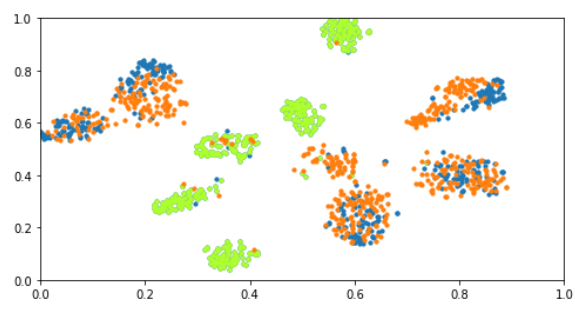

(c) IWAN

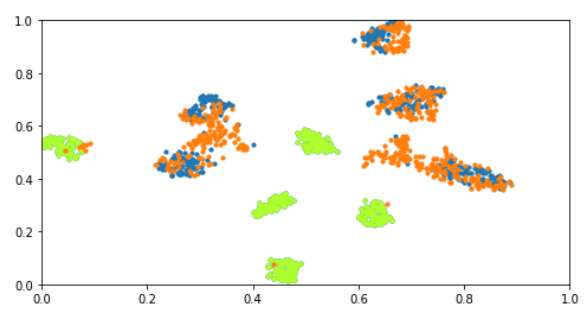

(e) DAPDA-DW

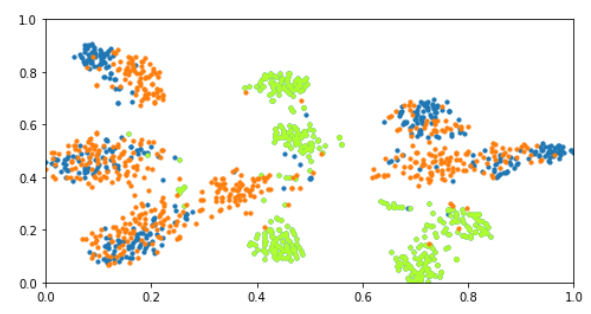

(b) RTN

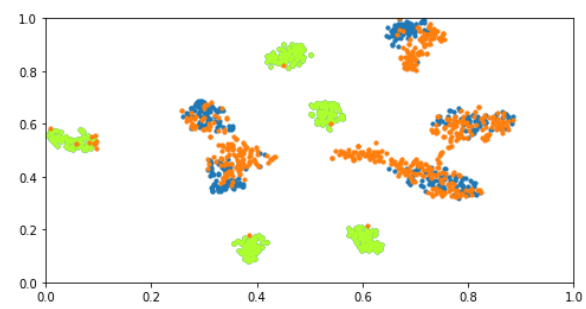

(d) DAPDA-CW

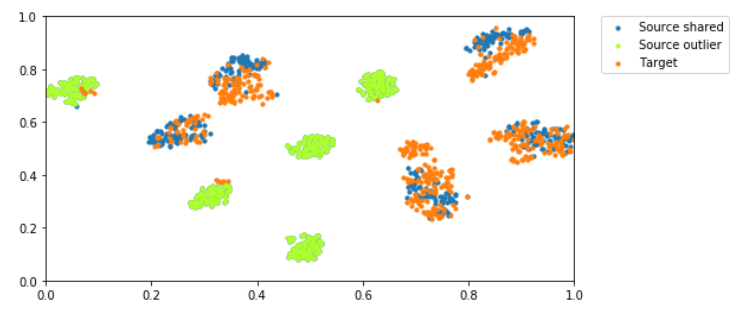

(f) DAPDA

Figure 17. The t-SNE visualization of learned features of compared and proposed methods for $\mathrm{A} 10 \rightarrow \mathrm{C} 5$ task on Office-Caltech dataset. The blue, green, and orange dots represent the source shared samples, source outlier samples, and target samples. The orange dots are expected to be aligned with the blue dots.

blue dots indicate source samples in shared classes and the green dots represent source samples in source outlier classes. The orange dots belong to the target domain. The adaptation is achieved if the orange dots are scarcely aligned with green dots but well-aligned with blue dots. In Figure 18, the dots with the same color belong to the same class for both domains. The alignment is performed effectively if intra-class dots have the same color and inter-class dots have different colors. To preserve the target domain structure, RTN and IWAN both use target domain entropy minimization strategies to encourage low-density separation 


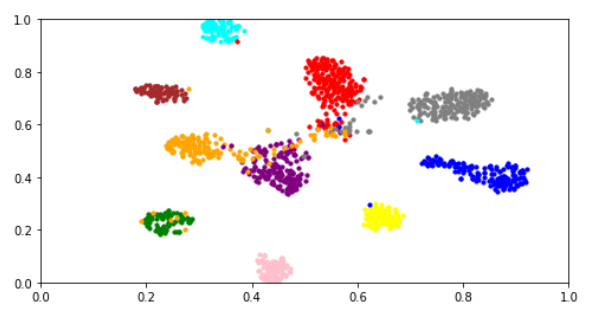

(a) AlexNet

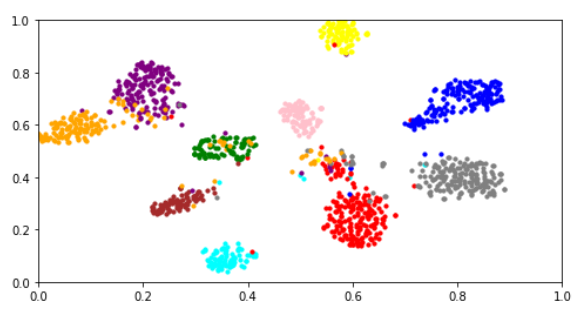

(c) IWAN

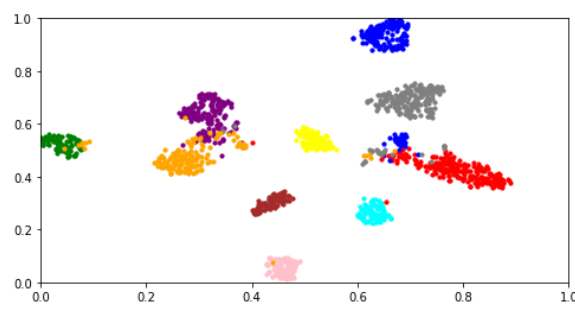

(e) DAPDA-DW

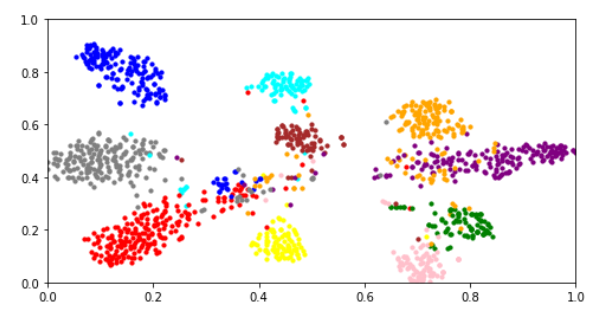

(b) RTN

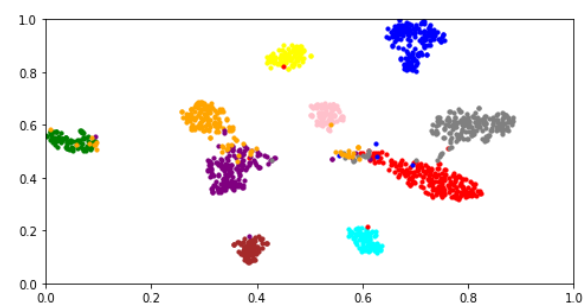

(d) DAPDA-CW

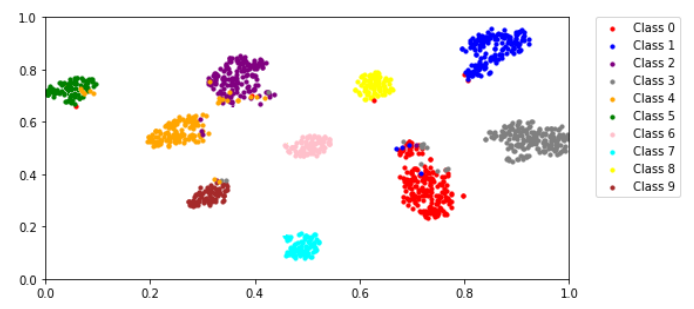

(f) DAPDA

Figure 18. The t-SNE visualization of learned features of compared and proposed methods for A10 $\rightarrow$ C5 task on Office-Caltech dataset. Each color represents a class. The dots with the same color are expected to be aligned.

among classes. Figure 17 (b)-(c) and Figure 18 (b)-(c) show the target samples are not spread to all classes but RTN cannot effectively ensure the intra-class compactness and IWAN misses more source outlier samples. The variants of our proposed methods only consider one style of alignment, i.e., DAPDA-CW focuses on class-wise alignment shown in Figure $17(\mathrm{~d})$, Figure $18(\mathrm{~d})$ and DAPDA-DW focuses on domain-wise alignment shown in Figure 17 (e), Figure 18 (e). Figure 17 (f) and Figure 18 (f) verify that our proposed DAPDA method selects out most source outlier samples and simultaneously ensures the inter-class separation as well as intra-class compactness. 
For parameter sensitivity, there are two tunable parameters: $\alpha$ and $\beta$, where $\alpha$ controls the balance between the intra-domain loss and the other losses, and $\beta$ controls the balance between the inter-domain loss and the other losses. We have conducted parameter sensitivity analysis on four transfer tasks: W31 $\rightarrow$ A10, A10 $\rightarrow \mathrm{C} 5$, Ar65 $\rightarrow \operatorname{Pr} 25$, and $\operatorname{Pr} 65 \rightarrow$ Cl25. From Figure 19, it can be seen that DAPDA could achieve good performance under a range of parameter values. At first, we run DAPDA as $\alpha$ varies from 0.001 to 10 when $\beta=1$. From Figure 19 (a), we can observe that the small $\alpha$ values would contribute to improving the accuracy and the too-large $\alpha$ values would degrade the performance. That is because it will weaken the effects of inter-domain loss term. Next, from Figure 19 (b), we evaluate DAPDA by varying $\beta$ from 0.001 to 10 with $\alpha$ fixed to 1 . It can be observed that small $\beta$ values would result in poor performance but reasonable $\beta$ values would enhance the accuracy. Note that DAPDA is more sensitive to $\beta$ than $\alpha$, and this guides us to determine $\alpha \in\left[\begin{array}{ll}0.01 & 5\end{array}\right]$ and $\beta \in\left[\begin{array}{ll}0.1 & 5\end{array}\right]$. In the experiments, we empirically set $\alpha=\beta=1$.

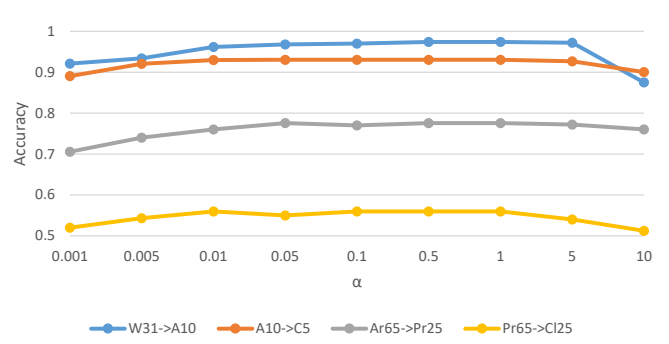

(a)

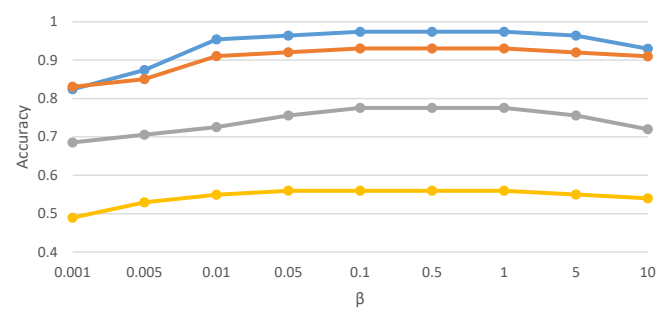

(b)

Figure 19. Parameter sensitivity on the task W31 $\rightarrow$ A10, A10 $\rightarrow$ C5, Ar65 $\rightarrow$ $\operatorname{Pr} 25$, and $\operatorname{Pr} 65 \rightarrow \mathrm{Cl} 25$. (a) parameter $\alpha$; (b) parameter $\beta$.

For convergence analysis, we give an example on the transfer task A10 $\rightarrow$ C5. Figure 20 (a) shows the changing trends of inter-domain loss, intra-domain loss, and the accuracy of soft labels with respect to a number of iterations. Our proposed DAPDA method converges fast on the test target data. We also plot the 
changing trend of the classifier loss, inter-domain loss, intra-domain loss, and the total loss with respect to a number of iterations in Figure 20 (b). As we can see, DAPDA converges to the lowest test error within 80 iterations.

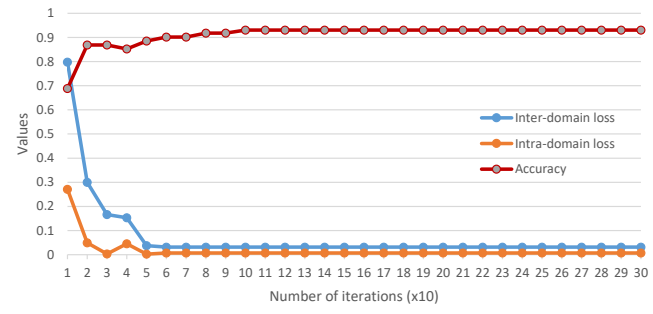

(a)

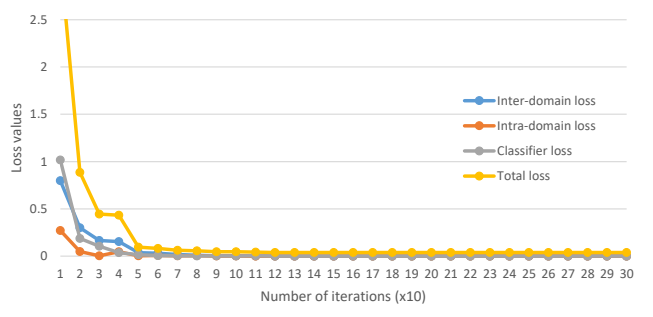

(b)

Figure 20. For the task A10 $\rightarrow$ C5, (a) Loss and accuracy values w.r.t. number of iterations (x10); (b) Loss values w.r.t. number of iterations (x10).

To demonstrate if the trained classifier $C_{y}$ can correctly reweight source classes according to whether they are in the shared label space, we give an example in Figure 21 of this response. It shows the histograms of class weights learned using ResNet-50, DANN, PADA, and DAPDA on task A31 $\rightarrow$ W10. The blue bins indicate source outlier classes, and the orange bins represent source shared classes in the shared label space. From Figure 21 (a) and (b), note that ResNet-50 and DANN can hardly select out outlier classes since there is not a sharp distinction between weights for outlier classes and that for shared classes. As shown in Figure 21 (c), we know that PADA can better distinguish the source outlier and shared classes. However, some weights for the shared classes are still below 0.5 such as Classes 10, 16, and 19. In the meanwhile, some outlier weights are higher than expected such as Classes 1, 7, 8, and 31. To compare with these methods, Figure $21(\mathrm{~d})$ shows the mean value of learned weights, i.e., $\left\{\frac{1}{n_{t}} \sum_{j=1}^{n_{t}} \widehat{q_{t, c_{k}}^{j}}\right\}_{k=1}^{K}$, which are the source class-level weights before normalization in DAPDA. We can notice that the weights for the shared classes are almost up to 1 and the weights for the outlier classes are close to 0 . The low weights for source outlier classes can greatly 
alleviate the raised negative transfer.

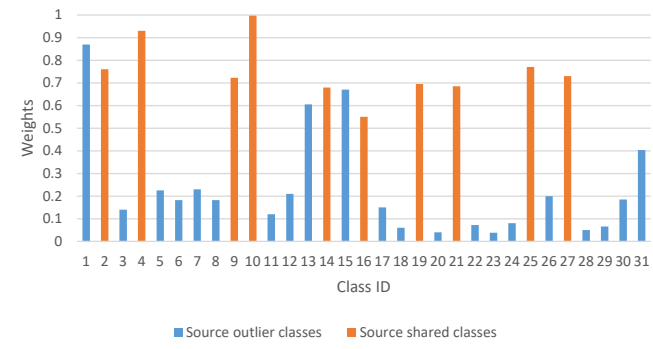

(a) ResNet-50

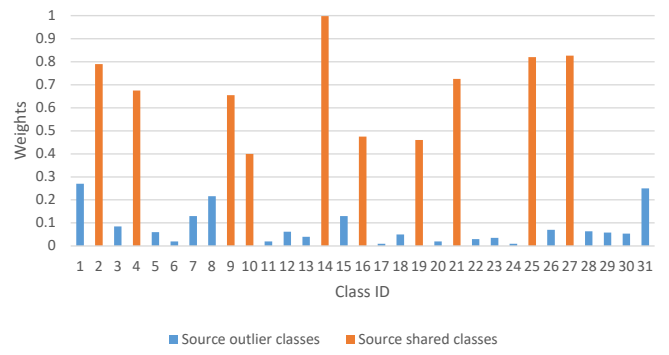

(c) PADA

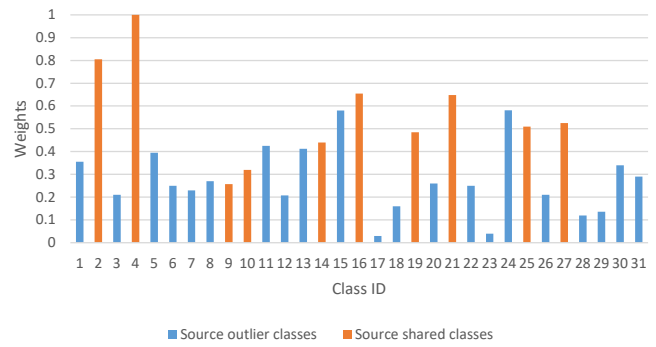

(b) DANN

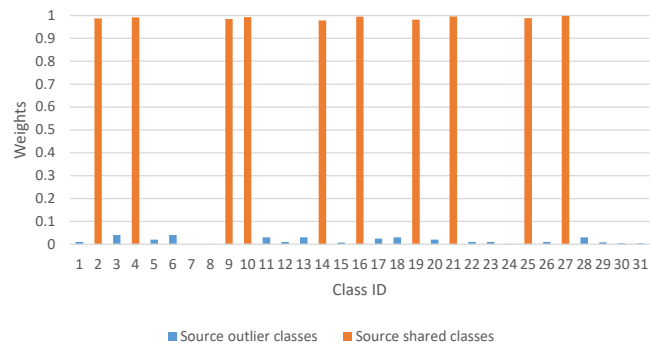

(d) DAPDA

Figure 21. For the task A31 $\rightarrow$ W10, histograms of class weights learned by (a) ResNet-50; (b) DANN; (c) PADA; (d) DAPDA.

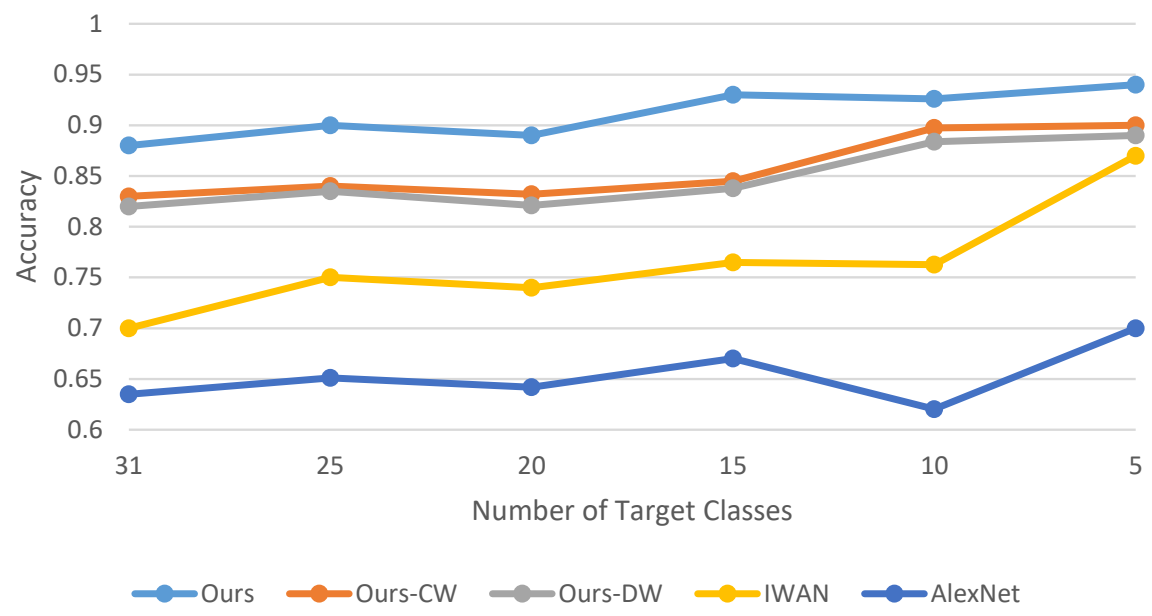

Figure 22. The accuracy curve of varying the number of target classes for A31 $\rightarrow$ W10 task using AlexNet as the baseline.

To evaluate the influence of the number of shared classes on performance, we also conduct experiments to compare classification accuracies by varying the 
number of target classes. Figure 22 shows that our proposed three methods outperform the AlexNet and IWAN to a large extent on $\mathrm{A} 31 \rightarrow \mathrm{W} 10$ transfer task using AlexNet as the baseline. As the number of target classes gets smaller, the performances have relatively better improvements. Our proposed DAPDA method shows an "even" best performance.

Table 14. Average accuracy (\%) of full domain adaptation tasks on Office-Caltech dataset (AlexNet)

\begin{tabular}{c|cccccccccccc|c}
\hline Methods & $\mathrm{A} \rightarrow \mathrm{C}$ & $\mathrm{A} \rightarrow \mathrm{D}$ & $\mathrm{A} \rightarrow \mathrm{W}$ & $\mathrm{C} \rightarrow \mathrm{A}$ & $\mathrm{C} \rightarrow \mathrm{D}$ & $\mathrm{C} \rightarrow \mathrm{W}$ & $\mathrm{D} \rightarrow \mathrm{A}$ & $\mathrm{D} \rightarrow \mathrm{C}$ & $\mathrm{D} \rightarrow \mathrm{W}$ & $\mathrm{W} \rightarrow \mathrm{A}$ & $\mathrm{W} \rightarrow \mathrm{C}$ & $\mathrm{W} \rightarrow \mathrm{D}$ & $\mathrm{AVG}$ \\
\hline DANN & 87.81 & 91.20 & 81.11 & 92.43 & 91.22 & 89.50 & 87.93 & 82.91 & 98.90 & 82.31 & 85.64 & $\mathbf{1 0 0 . 0}$ & 89.25 \\
DAN & 84.06 & 91.71 & 91.82 & 92.00 & 89.27 & 90.57 & 89.98 & 80.27 & 98.51 & 92.08 & 81.19 & $\mathbf{1 0 0 . 0}$ & 90.67 \\
DMM & 83.30 & 93.00 & 92.20 & 92.60 & 91.70 & 90.50 & 93.20 & 84.30 & 99.70 & 92.50 & 85.80 & $\mathbf{1 0 0 . 0}$ & 92.70 \\
WDGRL & 86.99 & 93.68 & 89.47 & 93.54 & 94.74 & 91.58 & 91.69 & 90.24 & 97.89 & 93.67 & 89.43 & $\mathbf{1 0 0 . 0}$ & 92.74 \\
\hline Ours-MMD & 90.16 & 96.53 & 90.24 & 93.72 & 93.63 & 90.51 & 93.88 & 88.10 & 97.60 & 93.55 & 87.92 & $\mathbf{1 0 0 . 0}$ & 92.99 \\
Ours-CORAL & 86.35 & 91.19 & 91.05 & 92.89 & 90.04 & 92.73 & 86.03 & 85.73 & 97.88 & 89.06 & 88.70 & $\mathbf{1 0 0 . 0}$ & 90.97 \\
DAPDA-N-EN & 90.50 & 98.23 & 98.91 & 94.34 & 94.71 & 94.71 & 94.30 & 90.16 & 99.02 & 96.63 & 89.54 & $\mathbf{1 0 0 . 0}$ & 95.08 \\
DAPDA & $\mathbf{9 2 . 2 7}$ & $\mathbf{9 8 . 1 9}$ & $\mathbf{9 9 . 2 5}$ & $\mathbf{9 4 . 4 2}$ & $\mathbf{9 4 . 8 3}$ & $\mathbf{9 4 . 7 6}$ & $\mathbf{9 5 . 0 0}$ & $\mathbf{9 0 . 3 3}$ & $\mathbf{9 9 . 1 0}$ & $\mathbf{9 6 . 4 0}$ & $\mathbf{9 0 . 2 4}$ & $\mathbf{1 0 0 . 0}$ & $\mathbf{9 5 . 4 0}$ \\
\hline
\end{tabular}

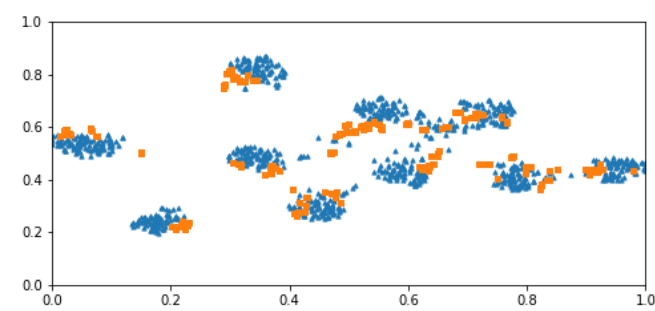

(a) DANN

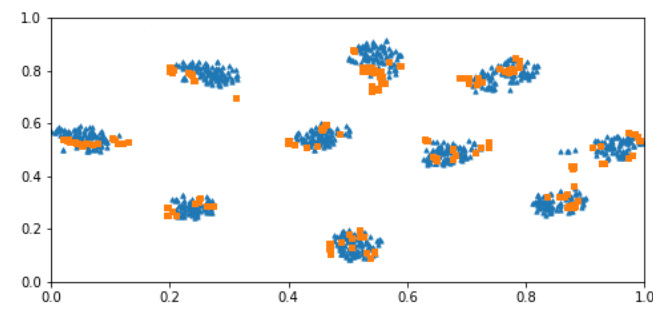

(c) Ours-CORAL

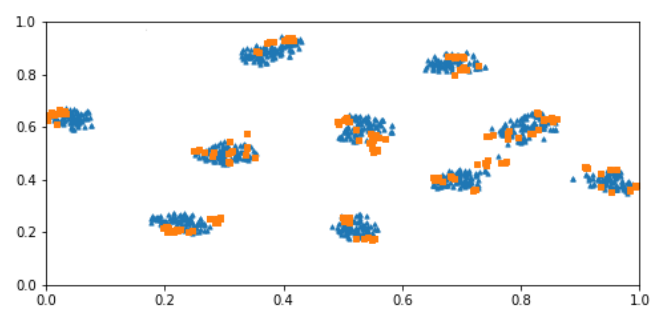

(b) Ours-MMD

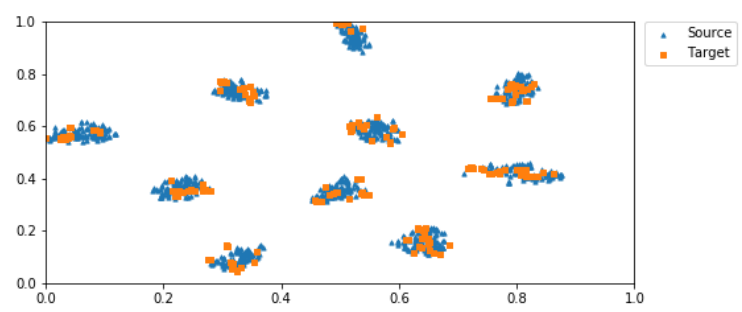

(d) DAPDA

Figure 23. The t-SNE visualization of learned features of compared and proposed methods for $\mathrm{A} \rightarrow \mathrm{C}$ task on Office-Caltech dataset. The blue and orange dots represent source and target samples, respectively. The orange dots are expected to be aligned with the blue dots.

To verify our proposed DAPDA on the full domain adaptation setting, we further conduct experiments on the Office-Caltech dataset. The average classifi- 


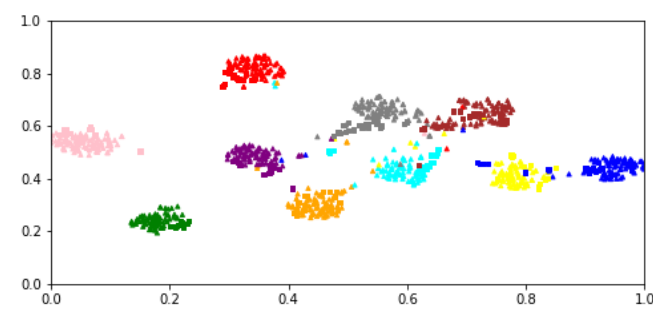

(a) DANN

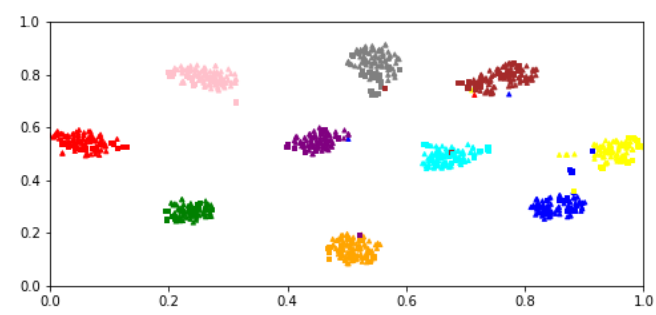

(c) Ours-CORAL

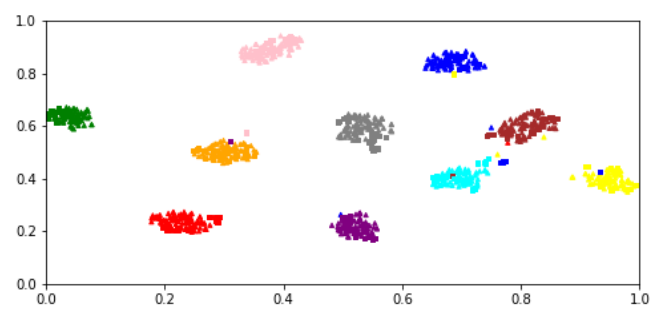

(b) Ours-MMD

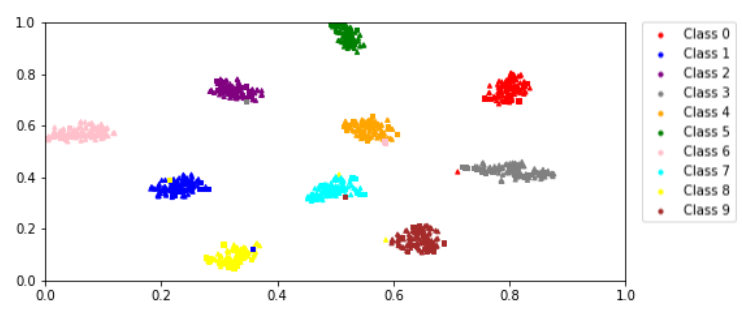

(d) DAPDA

Figure 24. The t-SNE visualization of learned features of compared and proposed methods for $\mathrm{A} \rightarrow \mathrm{C}$ task on Office-Caltech dataset. Each color represents a class. The dots with the same color are expected to be aligned.

cation accuracies are shown in Table 14, which manifests that DAPDA still has superiority in the standard full protocol. That is because we design DAPDA to not only align the source and target domains as much as possible, but also explore the class-invariant information in the shared label space of both domains. The results also show that DAPDA outperforms the Ours-MMD and Ours-CORAL such that the effectiveness of WD can be demonstrated. Moreover, we visualize the learned feature representations of several methods for $\mathrm{A} \rightarrow \mathrm{C}$ transfer task in Table 14. From Figure 23 and Figure 24, it can be observed that DAPDA better aligns source and target domains than other compared methods.

\subsection{Chapter Summary}

In this chapter, we propose a novel dual alignment method for partial domain adaptation (DAPDA). DAPDA can effectively select out source outlier samples and learn domain-invariant feature representations to explore latent structures un- 
der different data distributions across domains. The data distributions can be aligned in domain-wise and class-wise manners. The domain discrepancy can be effectively reduced using the metric of Wasserstein distance with gradient penalty. Experimental results on some datasets demonstrate that DAPDA outperforms several state-of-the-art partial domain adaptation methods. From feature visualization results, the great learning capability of DAPDA is manifested in capturing domain-invariant and target-discriminative representations. From the accuracy curve result, DAPDA shows even high performance. 


\section{CHAPTER 6}

\section{Conclusion and Future Work}

\subsection{Conclusion}

Multi-view data are semantically richer, more useful, however more complex compared to single-view data. Multi-view robust representation learning is a very important technique to be employed to extract valuable information from multiview data. In this dissertation, we consider several challenges in multi-view representation learning.

In chapter 2 , we develop a bipartite graph based multi-view clustering framework to learn the consensus representations across heterogeneous multiple views. This framework explores the consensus information by learning the uniform anchor points contained in different views. Moreover, multiple view-specific bipartite graphs and one view-consensus bipartite graph are constructed based on the similarity between data points and uniformed anchor points. An iterative optimization strategy is designed to update variables in the objective function. Thorough experiments have been conducted on both synthetic and real-world datasets. Results demonstrate the proposed framework outperforms the state-of-the-art models in most settings.

In chapter 3 , we design a joint partition and graph learning model for incomplete multi-view clustering to address the multi-view data with missing view information. This model jointly transforms local incomplete graphs into a unified partition space and employs it to learn a consensus graph. This model addresses both complete and incomplete multi-view clustering problems. An iterative optimization strategy is also adopted to update variables in the objective function. Extensive experiments have been conducted on real-world datasets with different missing settings. The effectiveness has been shown from the results. 
In chapter 4 , we propose an adversarial domain adaptation approach via category transfer. This approach aims to align the source and target domains at the class level. Specially, we minimize the discrepancies to learn category-invariant feature representations and obtain optimal predicted target labels. The experiments are conducted on several benchmark datasets. Simulation results in different transfer tasks show the effectiveness of the approach.

In chapter 5, we develop a dual alignment network for partial domain adaptation. It includes three key components: feature extractor, reweighting network, and dual alignment network. The source and target feature representations are extracted by the feature extractor. They are reweighted by reweighting network via exploring the role of each sample. The dual alignment network aligns source and target domains in both domain and class levels. This model addresses both full and

partial unsupervised domain adaptation problems. Simulation results demonstrate that this model has a better performance than the other baselines.

\subsection{Future Work}

We have shown the success of our methods for multi-view representation learning in image recognition. In my future research, I will continue the current research topics on multi-view learning as well as transfer learning, and expand their applications on wireless communications.

The cognitive radio, as a promising technology in wireless communications, has emerged to enhance spectrum utilization such that the scarcity problem caused by limited radio spectrum can be alleviated [109]. Specifically, cognitive radio senses radio frequency environment and allows unlicensed/secondary users (SUs) to opportunistically utilize the licensed spectrum, which is owned by licensed/primary users (PUs). To maximize spectrum utilization without interfering with the PUs' usages, SUs should have the ability of spectrum sensing to detect the presence of 
primary signals from PUs. In practice, the SUs often have no prior knowledge of the primary signals. Designing a blind sensing approach becomes an urgent issue. Base on the research experience, the following investigations are considered as future research opportunities:

Robust deep multi-view learning methods for automated modulation classification in wireless communication networks. Deep multi-view learning has demonstrated its success in information fusion from multiple data sources for a wide range of critical applications. In my previous work, I have designed a graph-based multi-view learning for cooperative spectrum sensing [110]. It can better fuse the information from different secondary users. Thus, it has the significant potential to be utilized for modulation detection and classification even in the presence of wireless communication environment noise. In future work, I will focus on deep multi-view learning architectures which can deal with the modulation classification in various environments.

Advanced transfer learning algorithms for spectrum sensing in cognitive radio networks. Transfer learning techniques have achieved great successes in various computer vision tasks, such as semi-supervised learning and unsupervised learning. In my previous work, I have developed a deep transfer spectrum sensing framework to detect the presence of arbitrary unknown signals in wireless communication networks [111]. The experiments demonstrated that the generalization ability and robustness of the framework are enhanced. Based on the research I have conducted about transfer learning, it is interesting to apply advanced transfer learning algorithms for wireless communication networks. 


\section{LIST OF REFERENCES}

[1] Z. Ding, M. Shao, and Y. Fu, "Robust multi-view representation: A unified perspective from multi-view learning to domain adaption." in IJCAI, 2018, pp. 5434-5440.

[2] S. Bickel and T. Scheffer, "Multi-view clustering." in ICDM, vol. 4, no. 2004, 2004, pp. 19-26.

[3] Y. Li, M. Yang, and Z. Zhang, "A survey of multi-view representation learning," IEEE transactions on knowledge and data engineering, vol. 31, no. 10, pp. 1863-1883, 2018.

[4] L. Li, H. He, J. Li, and G. Yang, "Adversarial domain adaptation via category transfer," in 2019 International Joint Conference on Neural Networks (IJCNN). IEEE, 2019, pp. 1-8.

[5] L. Li, Z. Wan, and H. He, "Dual alignment for partial domain adaptation," IEEE Transactions on Cybernetics, pp. 1-13, 2020.

[6] Z. Wan and H. He, "Answernet: Learning to answer questions," IEEE Transactions on Big Data, vol. 5, no. 4, pp. 540-549, 2019.

[7] S. Sun, "A survey of multi-view machine learning," Neural computing and applications, vol. 23, no. 7-8, pp. 2031-2038, 2013.

[8] X. Cai, F. Nie, and H. Huang, "Multi-view k-means clustering on big data," in Twenty-Third International Joint conference on artificial intelligence, 2013.

[9] J. Liu, C. Wang, J. Gao, and J. Han, "Multi-view clustering via joint nonnegative matrix factorization," in Proceedings of the 2013 SIAM International Conference on Data Mining. SIAM, 2013, pp. 252-260.

[10] G. Tzortzis and A. Likas, "Kernel-based weighted multi-view clustering," in 2012 IEEE 12th international conference on data mining. IEEE, 2012, pp. 675-684.

[11] C.-D. Wang, J.-H. Lai, and S. Y. Philip, "Multi-view clustering based on belief propagation," IEEE Transactions on Knowledge and Data Engineering, vol. 28, no. 4, pp. 1007-1021, 2015.

[12] X. Cao, C. Zhang, H. Fu, S. Liu, and H. Zhang, "Diversity-induced multiview subspace clustering," in Proceedings of the IEEE conference on computer vision and pattern recognition, 2015, pp. 586-594. 
[13] X. Zhu, S. Zhang, W. He, R. Hu, C. Lei, and P. Zhu, "One-step multi-view spectral clustering," IEEE Transactions on Knowledge and Data Engineering, vol. 31, no. 10, pp. 2022-2034, 2018.

[14] A. Kumar and H. Daumé, "A co-training approach for multi-view spectral clustering," in Proceedings of the 28th International Conference on Machine Learning (ICML-11), 2011, pp. 393-400.

[15] S. Huang, Z. Xu, I. W. Tsang, and Z. Kang, "Auto-weighted multi-view co-clustering with bipartite graphs," Information Sciences, 2019.

[16] X. Zhu, S. Zhang, Y. Li, J. Zhang, L. Yang, and Y. Fang, "Low-rank sparse subspace for spectral clustering," IEEE Transactions on Knowledge and Data Engineering, 2018.

[17] D. Xie, X. Zhang, Q. Gao, J. Han, S. Xiao, and X. Gao, "Multiview clustering by joint latent representation and similarity learning," IEEE transactions on cybernetics, 2019.

[18] F. Nie, G. Cai, and X. Li, "Multi-view clustering and semi-supervised classification with adaptive neighbours," in Thirty-First AAAI Conference on Artificial Intelligence, 2017.

[19] K. Zhan, C. Zhang, J. Guan, and J. Wang, "Graph learning for multiview clustering," IEEE transactions on cybernetics, vol. 48, no. 10, pp. 2887-2895, 2017.

[20] H. Xiao, Y. Chen, and X. Shi, "Knowledge graph embedding based on multiview clustering framework," IEEE Transactions on Knowledge and Data Engineering, 2019.

[21] R. Wang, F. Nie, Z. Wang, H. Hu, and X. Li, "Parameter-free weighted multiview projected clustering with structured graph learning," IEEE Transactions on Knowledge and Data Engineering, 2019.

[22] Q. Chen, Y. Liu, Z. Wang, I. Wassell, and K. Chetty, "Re-weighted adversarial adaptation network for unsupervised domain adaptation," in CVPR, June 2018.

[23] J. Shen, Y. Qu, W. Zhang, and Y. Yu, "Wasserstein distance guided representation learning for domain adaptation," in Thirty-Second AAAI Conference on Artificial Intelligence, 2018.

[24] Y. Cao, M. Long, and J. Wang, "Unsupervised domain adaptation with distribution matching machines," in Thirty-Second AAAI Conference on Artificial Intelligence, 2018. 
[25] M. Long, Y. Cao, J. Wang, and M. I. Jordan, "Learning transferable features with deep adaptation networks," in Proceedings of the 32nd International Conference on International Conference on Machine Learning, 2015, pp. $97-105$.

[26] E. Tzeng, J. Hoffman, T. Darrell, and K. Saenko, "Simultaneous deep transfer across domains and tasks," in ICCV, 2015, pp. 4068-4076.

[27] A. Rozantsev, M. Salzmann, and P. Fua, "Beyond sharing weights for deep domain adaptation," IEEE Transactions on Pattern Analysis and Machine Intelligence (TPAMI), 2018.

[28] K. Saito, K. Watanabe, Y. Ushiku, and T. Harada, "Maximum classifier discrepancy for unsupervised domain adaptation," in CVPR, June 2018.

[29] J. Li, K. Lu, Z. Huang, L. Zhu, and H. T. Shen, "Heterogeneous domain adaptation through progressive alignment," IEEE transactions on neural networks and learning systems, vol. 30, no. 5, pp. 1381-1391, 2018.

[30] J. Zhang, Z. Ding, W. Li, and P. Ogunbona, "Importance weighted adversarial nets for partial domain adaptation," in Proceedings of the IEEE Conference on Computer Vision and Pattern Recognition, 2018, pp. 8156-8164.

[31] Z. Cao, M. Long, J. Wang, and M. I. Jordan, "Partial transfer learning with selective adversarial networks," in Proceedings of the IEEE Conference on Computer Vision and Pattern Recognition, 2018, pp. 2724-2732.

[32] Z. Cao, L. Ma, M. Long, and J. Wang, "Partial adversarial domain adaptation," in Proceedings of the European Conference on Computer Vision (ECCV), 2018, pp. 135-150.

[33] Z. Cao, K. You, M. Long, J. Wang, and Q. Yang, "Learning to transfer examples for partial domain adaptation," in Proceedings of the IEEE Conference on Computer Vision and Pattern Recognition, 2019, pp. 2985-2994.

[34] H. Tong, J. He, M. Li, C. Zhang, and W.-Y. Ma, "Graph based multimodality learning," in Proceedings of the 13th annual ACM international conference on Multimedia. ACM, 2005, pp. 862-871.

[35] H. Wang, Y. Yang, B. Liu, and H. Fujita, "A study of graph-based system for multi-view clustering," Knowledge-Based Systems, vol. 163, pp. 1009-1019, 2019.

[36] Z. Kang, H. Pan, S. C. Hoi, and Z. Xu, "Robust graph learning from noisy data," IEEE transactions on cybernetics, 2019. 
[37] J. Zhao, L. Li, F. Deng, H. He, and J. Chen, "Discriminant geometrical and statistical alignment with density peaks for domain adaptation," IEEE Transactions on Cybernetics, 2020.

[38] H. Wang, Y. Yang, and B. Liu, "Gmc: Graph-based multi-view clustering," IEEE Transactions on Knowledge and Data Engineering, vol. 32, no. 6, pp. 1116-1129, 2019.

[39] Y. Li, F. Nie, H. Huang, and J. Huang, "Large-scale multi-view spectral clustering via bipartite graph," in Proceedings of the twenty-ninth AAAI conference on artificial intelligence, 2015, pp. 2750-2756.

[40] Z. Wan, H. He, and B. Tang, "A generative model for sparse hyperparameter determination," IEEE Transactions on Big Data, vol. 4, no. 1, pp. 2-10, 2018.

[41] L. Li and H. He, "Bipartite graph based multi-view clustering," IEEE Transactions on Knowledge and Data Engineering, pp. 1-1, 2020.

[42] T. He, Y. Liu, T. H. Ko, K. C. Chan, and Y.-S. Ong, "Contextual correlation preserving multiview featured graph clustering," IEEE transactions on cybernetics, 2019.

[43] F. Nie, G. Cai, J. Li, and X. Li, "Auto-weighted multi-view learning for image clustering and semi-supervised classification," IEEE Transactions on Image Processing, vol. 27, no. 3, pp. 1501-1511, 2017.

[44] F. Nie, C.-L. Wang, and X. Li, "K-multiple-means: A multiple-means clustering method with specified k clusters," in Proceedings of the 25th ACM SIGKDD International Conference on Knowledge Discovery \& Data Mining. ACM, 2019, pp. 959-967.

[45] F. R. Chung and F. C. Graham, Spectral graph theory. American Mathematical Soc., 1997, no. 92.

[46] K. Fan, "On a theorem of weyl concerning eigenvalues of linear transformations i," Proceedings of the National Academy of Sciences of the United States of America, vol. 35, no. 11, p. 652, 1949.

[47] S. Boyd, S. P. Boyd, and L. Vandenberghe, Convex optimization. Cambridge university press, 2004.

[48] S. Huang, Z. Xu, I. W. Tsang, and Z. Kang, "Auto-weighted multi-view coclustering with bipartite graphs," Information Sciences, vol. 512, pp. 18-30, 2020 .

[49] W. Zhuge, F. Nie, C. Hou, and D. Yi, "Unsupervised single and multiple views feature extraction with structured graph," IEEE Transactions on Knowledge and Data Engineering, vol. 29, no. 10, pp. 2347-2359, 2017. 
[50] H. Wang, Y. Yang, and T. Li, "Multi-view clustering via concept factorization with local manifold regularization," in 2016 IEEE 16th International Conference on Data Mining (ICDM). IEEE, 2016, pp. 1245-1250.

[51] M. Brbić and I. Kopriva, "Multi-view low-rank sparse subspace clustering," Pattern Recognition, vol. 73, pp. 247-258, 2018.

[52] S. Li, L. Li, J. Yan, and H. He, "Sde: A novel clustering framework based on sparsity-density entropy," IEEE Transactions on Knowledge and Data Engineering, vol. 30, no. 8, pp. 1575-1587, 2018.

[53] L. Li, H. He, and J. Li, "Entropy-based sampling approaches for multiclass imbalanced problems," IEEE Transactions on Knowledge and Data Engineering, 2019.

[54] J. Wen, Y. Xu, and H. Liu, "Incomplete multiview spectral clustering with adaptive graph learning," IEEE transactions on cybernetics, 2018.

[55] X. Liu, X. Zhu, M. Li, L. Wang, E. Zhu, T. Liu, M. Kloft, D. Shen, J. Yin, and W. Gao, "Multiple kernel k-means with incomplete kernels," IEEE transactions on pattern analysis and machine intelligence, 2019.

[56] W. Zhou, H. Wang, and Y. Yang, "Consensus graph learning for incomplete multi-view clustering," in Pacific-Asia Conference on Knowledge Discovery and Data Mining. Springer, 2019, pp. 529-540.

[57] S.-Y. Li, Y. Jiang, and Z.-H. Zhou, "Partial multi-view clustering," in Twenty-Eighth AAAI Conference on Artificial Intelligence, 2014.

[58] H. Zhao, H. Liu, and Y. Fu, "Incomplete multi-modal visual data grouping." in IJCAI, 2016, pp. 2392-2398.

[59] M. Hu and S. Chen, "Doubly aligned incomplete multi-view clustering," arXiv preprint arXiv:1903.02785, 2019.

[60] L. Li, Z. Wan, and H. He, "Incomplete multi-view clustering with joint partition and graph learning," IEEE Transactions on Knowledge and Data Engineering, 2020 (under revision).

[61] J. Wen, Z. Zhang, Y. Xu, and Z. Zhong, "Incomplete multi-view clustering via graph regularized matrix factorization," in Proceedings of the European Conference on Computer Vision (ECCV), 2018, pp. 0-0.

[62] H. Wang, L. Zong, B. Liu, Y. Yang, and W. Zhou, "Spectral perturbation meets incomplete multi-view data," arXiv preprint arXiv:1906.00098, 2019.

[63] Z. Tao, H. Liu, S. Li, Z. Ding, and Y. Fu, "From ensemble clustering to multi-view clustering," in IJCAI, 2017. 
[64] Z. Kang, Z. Guo, S. Huang, S. Wang, W. Chen, Y. Su, and Z. Xu, "Multiple partitions aligned clustering," arXiv preprint arXiv:1909.06008, 2019.

[65] Z. Kang, X. Zhao, C. Peng, H. Zhu, J. T. Zhou, X. Peng, W. Chen, and Z. Xu, "Partition level multiview subspace clustering," Neural Networks, vol. 122, pp. 279-288, 2020.

[66] A. Y. Ng, M. I. Jordan, and Y. Weiss, "On spectral clustering analysis and an algorithm," in Advances in neural information processing systems, 2002, pp. 849-856.

[67] W. Shao, L. He, and S. Y. Philip, "Multiple incomplete views clustering via weighted nonnegative matrix factorization with 12,1 regularization," in Joint European Conference on Machine Learning and Knowledge Discovery in Databases. Springer, 2015, pp. 318-334.

[68] I. Goodfellow, J. Pouget-Abadie, M. Mirza, B. Xu, D. Warde-Farley, S. Ozair, A. Courville, and Y. Bengio, "Generative adversarial nets," in Advances in neural information processing systems, 2014, pp. 2672-2680.

[69] E. Tzeng, J. Hoffman, K. Saenko, and T. Darrell, "Adversarial discriminative domain adaptation," in Proceedings of the IEEE Conference on Computer Vision and Pattern Recognition, 2017, pp. 7167-7176.

[70] R. Volpi, P. Morerio, S. Savarese, and V. Murino, "Adversarial feature augmentation for unsupervised domain adaptation," in CVPR, 2017.

[71] Z. Pei, Z. Cao, M. Long, and J. Wang, "Multi-adversarial domain adaptation," in AAAI Conference on Artificial Intelligence, 2018.

[72] L. Li, H. He, J. Li, and G. Yang, "Adversarial domain adaptation via category transfer," in 2019 International Joint Conference on Neural Networks (IJCNN), 2019, pp. 1-8.

[73] M. Arjovsky, S. Chintala, and L. Bottou, "Wasserstein gan," arXiv preprint arXiv:1701.07875, 2017.

[74] X. Wang, H. He, and L. Li, "A hierarchical deep domain adaptation approach for fault diagnosis of power plant thermal system," IEEE Transactions on Industrial Informatics, vol. 15, no. 9, pp. 5139-5148, 2019.

[75] J. Zhao, L. Li, F. Deng, H. He, and J. Chen, "Discriminant geometrical and statistical alignment with density peaks for domain adaptation," IEEE Transactions on Cybernetics, pp. 1-14, 2020.

[76] Y. Ganin, E. Ustinova, H. Ajakan, P. Germain, H. Larochelle, F. Laviolette, M. Marchand, and V. Lempitsky, "Domain-adversarial training of neural networks," The Journal of Machine Learning Research, vol. 17, no. 1, pp. 2096-2030, 2016. 
[77] Z. Wan, L. Li, H. Li, H. He, and Z. Ni, "One-shot unsupervised domain adaptation for object detection," in 2020 International Joint Conference on Neural Networks (IJCNN), 2020, pp. 1-8.

[78] A. Gretton, K. M. Borgwardt, M. J. Rasch, B. Schölkopf, and A. Smola, "A kernel two-sample test," Journal of Machine Learning Research, vol. 13, no. Mar, pp. 723-773, 2012.

[79] E. Tzeng, J. Hoffman, N. Zhang, K. Saenko, and T. Darrell, "Deep domain confusion: Maximizing for domain invariance," in CVPR, 2014.

[80] B. Sun, J. Feng, and K. Saenko, "Return of frustratingly easy domain adaptation," in Thirtieth AAAI Conference on Artificial Intelligence, 2016.

[81] B. Sun and K. Saenko, "Deep coral: Correlation alignment for deep domain adaptation," in ECCV. Springer, 2016, pp. 443-450.

[82] H. Ajakan, P. Germain, H. Larochelle, F. Laviolette, and M. Marchand, "Domain-adversarial neural networks," arXiv preprint arXiv:1412.4446, 2014.

[83] J. Zhang, Z. Ding, W. Li, and P. Ogunbona, "Importance weighted adversarial nets for partial domain adaptation," in CVPR, June 2018.

[84] N. Courty, R. Flamary, A. Habrard, and A. Rakotomamonjy, "Joint distribution optimal transportation for domain adaptation," in NIPS, 2017, pp. 3730-3739.

[85] B. Bhushan Damodaran, B. Kellenberger, R. Flamary, D. Tuia, and N. Courty, "Deepjdot: Deep joint distribution optimal transport for unsupervised domain adaptation," in Proceedings of the European Conference on Computer Vision (ECCV), 2018, pp. 447-463.

[86] I. Redko, A. Habrard, and M. Sebban, "Theoretical analysis of domain adaptation with optimal transport," in Machine Learning and Knowledge Discovery in Databases. Springer, Cham, 2017, pp. 737-753.

[87] I. Gulrajani, F. Ahmed, M. Arjovsky, V. Dumoulin, and A. C. Courville, "Improved training of wasserstein gans," in Advances in neural information processing systems, 2017 , pp. 5767-5777.

[88] C. Villani, Optimal transport: old and new. Springer Science \& Business Media, 2008, vol. 338.

[89] Y. LeCun, L. Bottou, Y. Bengio, and P. Haffner, "Gradient-based learning applied to document recognition," Proceedings of the IEEE, vol. 86, no. 11, pp. 2278-2324, 1998. 
[90] J. J. Hull, "A database for handwritten text recognition research," IEEE Transactions on pattern analysis and machine intelligence, vol. 16, no. 5, pp. 550-554, 1994.

[91] Y. Netzer, T. Wang, A. Coates, A. Bissacco, B. Wu, and A. Y. Ng, "Reading digits in natural images with unsupervised feature learning," in NIPS workshop on deep learning and unsupervised feature learning, vol. 2011, no. 2, 2011, p. 5 .

[92] B. Gong, Y. Shi, F. Sha, and K. Grauman, "Geodesic flow kernel for unsupervised domain adaptation," in 2012 IEEE Conference on Computer Vision and Pattern Recognition. IEEE, 2012, pp. 2066-2073.

[93] R. Aljundi, R. Emonet, D. Muselet, and M. Sebban, "Landmarks-based kernelized subspace alignment for unsupervised domain adaptation," in $C V P R$, 2015, pp. 56-63.

[94] J. Donahue, Y. Jia, O. Vinyals, J. Hoffman, N. Zhang, E. Tzeng, and T. Darrell, "Decaf: A deep convolutional activation feature for generic visual recognition," in International conference on machine learning, 2014, pp. 647-655.

[95] E. Zhong, W. Fan, Q. Yang, O. Verscheure, and J. Ren, "Cross validation framework to choose amongst models and datasets for transfer learning," in ECML/PKDD. Springer, 2010, pp. 547-562.

[96] M. Long, J. Wang, Y. Cao, J. Sun, and S. Y. Philip, "Deep learning of transferable representation for scalable domain adaptation," IEEE Transactions on Knowledge and Data Engineering, vol. 28, no. 8, pp. 2027-2040, 2016.

[97] L. Li, Z. Wan, and H. He, "Dual alignment for partial domain adaptation," IEEE Transactions on Cybernetics, pp. 1-13, 2020.

[98] L. Li, H. He, J. Li, and W. Li, "EDOS: Entropy difference-based oversampling approach for imbalanced learning," in 2018 International Joint Conference on Neural Networks (IJCNN). IEEE, 2018, pp. 1-8.

[99] Z. Wan, H. Li, H. He, and D. Prokhorov, "Model-free real-time ev charging scheduling based on deep reinforcement learning," IEEE Transactions on Smart Grid, vol. 10, no. 5, pp. 5246-5257, 2019.

[100] Z. Wan, C. Jiang, M. Fahad, Z. Ni, Y. Guo, and H. He, "Robot-assisted pedestrian regulation based on deep reinforcement learning," IEEE Transactions on Cybernetics, pp. 1-14, 2018.

[101] M. Long, H. Zhu, J. Wang, and M. I. Jordan, "Unsupervised domain adaptation with residual transfer networks," in Advances in Neural Information Processing Systems, 2016, pp. 136-144. 
[102] H. Narayanan and S. Mitter, "Sample complexity of testing the manifold hypothesis," in Advances in Neural Information Processing Systems, 2010, pp. 1786-1794.

[103] Y. Grandvalet and Y. Bengio, "Semi-supervised learning by entropy minimization," in Advances in neural information processing systems, 2005, pp. $529-536$.

[104] K. Saenko, B. Kulis, M. Fritz, and T. Darrell, "Adapting visual category models to new domains," in European conference on computer vision. Springer, 2010, pp. 213-226.

[105] H. Venkateswara, J. Eusebio, S. Chakraborty, and S. Panchanathan, "Deep hashing network for unsupervised domain adaptation," in Proceedings of the IEEE Conference on Computer Vision and Pattern Recognition, 2017, pp. $5018-5027$.

[106] A. Krizhevsky, I. Sutskever, and G. E. Hinton, "Imagenet classification with deep convolutional neural networks," in Advances in neural information processing systems, 2012, pp. 1097-1105.

[107] K. He, X. Zhang, S. Ren, and J. Sun, "Deep residual learning for image recognition," in Proceedings of the IEEE conference on computer vision and pattern recognition, 2016, pp. 770-778.

[108] M. Long, H. Zhu, J. Wang, and M. I. Jordan, "Deep transfer learning with joint adaptation networks," in Proceedings of the 34 th International Conference on Machine Learning, 2017, pp. 2208-2217.

[109] H. Jiang, L. Li, H. He, and L. Liu, "Evolutionary search for energy-efficient distributed cooperative spectrum sensing," in 2020 International Conference on Computing, Networking and Communications (ICNC), 2020, pp. 567-571.

[110] L. Li, H. Jiang, and H. He, "Graph-based multi-view learning for cooperative spectrum sensing," in 2021 International Joint Conference on Neural Networks (IJCNN), 2021, pp. 1-8.

[111] L. Li, H. Jiang, and H. He, "Deep transfer cooperative sensing in cognitive radio," IEEE Wireless Communications Letters, pp. 1-1, 2021. 


\section{BIBLIOGRAPHY}

Akyildiz, I. F., Lee, W.-Y., Vuran, M. C., and Mohanty, S., "Next generation/dynamic spectrum access/cognitive radio wireless networks: A survey," Computer networks, vol. 50, no. 13, pp. 2127-2159, 2006.

Akyildiz, I. F., Lo, B. F., and Balakrishnan, R., "Cooperative spectrum sensing in cognitive radio networks: A survey," Physical communication, vol. 4, no. 1, pp. 40-62, 2011.

Alnwaimi, G., Arshad, K., and Moessner, K., "Dynamic spectrum allocation algorithm with interference management in co-existing networks," IEEE Communications Letters, vol. 15, no. 9, pp. 932-934, 2011.

Andrews, J. G., Claussen, H., Dohler, M., Rangan, S., and Reed, M. C., "Femtocells: Past, present, and future," IEEE Journal on Selected Areas in communications, vol. 30, no. 3, pp. 497-508, 2012.

Arulkumaran, K., Deisenroth, M. P., Brundage, M., and Bharath, A. A., "Deep reinforcement learning: A brief survey," IEEE Signal Processing Magazine, vol. 34, no. 6, pp. 26-38, 2017.

Asadi, A., Wang, Q., and Mancuso, V., "A survey on device-to-device communication in cellular networks," IEEE Communications Surveys 83 Tutorials, vol. 16, no. 4, pp. 1801-1819, 2014.

Axell, E., Leus, G., Larsson, E. G., and Poor, H. V., "Spectrum sensing for cognitive radio: State-of-the-art and recent advances," IEEE signal processing magazine, vol. 29, no. 3, pp. 101-116, 2012.

Azarafrooz, M. and Chandramouli, R., "Distributed learning in secondary spectrum sharing graphical game," in 2011 IEEE Global Telecommunications Conference-GLOBECOM 2011. IEEE, 2011, pp. 1-5.

Belkin, M., Niyogi, P., and Sindhwani, V., "Manifold regularization: A geometric framework for learning from labeled and unlabeled examples," Journal of machine learning research, vol. 7, no. Nov, pp. 2399-2434, 2006.

Bello, I., Pham, H., Le, Q. V., Norouzi, M., and Bengio, S., "Neural combinatorial optimization with reinforcement learning," arXiv preprint arXiv:1611.09940, 2016.

Cai, H., Zheng, V. W., and Chang, K. C.-C., "A comprehensive survey of graph embedding: Problems, techniques, and applications," IEEE Transactions on Knowledge and Data Engineering, vol. 30, no. 9, pp. 1616-1637, 2018. 
Cai, Q., Chen, S., Li, X., Hu, N., He, H., Yao, Y.-D., and Mitola, J., "An integrated incremental self-organizing map and hierarchical neural network approach for cognitive radio learning," in The 2010 International Joint Conference on Neural Networks (IJCNN). IEEE, 2010, pp. 1-6.

Chandhar, P. and Das, S. S., "Area spectral efficiency of co-channel deployed ofdma femtocell networks," IEEE Transactions on Wireless Communications, vol. 13, no. 7, pp. 3524-3538, 2014.

Chandrasekhar, V. and Andrews, J. G., "Uplink capacity and interference avoidance for two-tier femtocell networks," arXiv preprint cs/0702132, 2007.

Chandrasekhar, V. and Andrews, J. G., "Spectrum allocation in tiered cellular networks," IEEE Transactions on Communications, vol. 57, no. 10, 2009.

Chandrasekhar, V., Andrews, J. G., and Gatherer, A., "Femtocell networks: a survey," IEEE Communications magazine, vol. 46, no. 9, 2008.

Chang, H.-H., Song, H., Yi, Y., Zhang, J., He, H., and Liu, L., "Distributive dynamic spectrum access through deep reinforcement learning: A reservoir computing based approach," IEEE Internet of Things Journal, 2018.

Chen, X. and Huang, J., "Distributed spectrum access with spatial reuse," IEEE Journal on Selected Areas in Communications, vol. 31, no. 3, pp. 593-603, 2013.

Cheng, P., Deng, R., and Chen, J., "Energy-efficient cooperative spectrum sensing in sensor-aided cognitive radio networks," IEEE Wireless Communications, vol. 19, no. 6, pp. 100-105, 2012.

Cheng, S.-M., Lien, S.-Y., Chu, F.-S., and Chen, K.-C., "On exploiting cognitive radio to mitigate interference in macro/femto heterogeneous networks," IEEE Wireless Communications, vol. 18, no. 3, 2011.

Cplex, I. I., "V12. 1: User's manual for cplex," International Business Machines Corporation, vol. 46, no. 53, p. 157, 2009.

Dai, H., Dai, B., and Song, L., "Discriminative embeddings of latent variable models for structured data," in International Conference on Machine Learning, 2016, pp. 2702-2711.

Davis, L., "Handbook of genetic algorithms," 1991.

Deng, Z., Dong, Y., and Zhu, J., "Batch virtual adversarial training for graph convolutional networks," arXiv preprint arXiv:1902.09192, 2019.

Dohler, M., Heath, R. W., Lozano, A., Papadias, C. B., and Valenzuela, R. A., "Is the phy layer dead?" IEEE Communications Magazine, vol. 49, no. 4, 2011. 
Doppler, K., Rinne, M., Wijting, C., Ribeiro, C. B., and Hugl, K., "Device-todevice communication as an underlay to lte-advanced networks," IEEE communications magazine, vol. 47, no. 12, pp. 42-49, 2009.

Etkin, R., Parekh, A., and Tse, D., "Spectrum sharing for unlicensed bands," IEEE Journal on selected areas in communications, vol. 25, no. 3, pp. 517-528, 2007.

Fan, C., Li, B., Zhao, C., Guo, W., and Liang, Y.-C., "Learning-based spectrum sharing and spatial reuse in mm-wave ultradense networks," IEEE Transactions on Vehicular Technology, vol. 67, no. 6, pp. 4954-4968, 2018.

Feng, D., Lu, L., Yi, Y.-W., Li, G. Y., Feng, G., and Li, S., "Qos-aware resource allocation for device-to-device communications with channel uncertainty," IEEE Transactions on Vehicular Technology, vol. 65, no. 8, pp. 6051-6062, 2015.

Feng, F., He, X., Tang, J., and Chua, T.-S., "Graph adversarial training: Dynamically regularizing based on graph structure," IEEE Transactions on Knowledge and Data Engineering, 2019.

Fette, B. A., Cognitive radio technology. Elsevier, 2006.

Fodor, G., Dahlman, E., Mildh, G., Parkvall, S., Reider, N., Miklós, G., and Turányi, Z., "Design aspects of network assisted device-to-device communications," IEEE Communications Magazine, vol. 50, no. 3, pp. 170-177, 2012.

Force, F. S. P. T., "Report of the spectrum efficiency working group, nov. 2002," URL http://www. fcc. gov/sptf/reports. html.

Ganesan, G. and Li, Y., "Cooperative spectrum sensing in cognitive radio, part ii: multiuser networks," IEEE Transactions on wireless communications, vol. 6 , no. 6, pp. 2214-2222, 2007.

Gen, M., Cheng, R., and Wang, D., "Genetic algorithms for solving shortest path problems," in Proceedings of 1997 IEEE International Conference on Evolutionary Computation (ICEC'97). IEEE, 1997, pp. 401-406.

Haykin, S., Thomson, D. J., and Reed, J. H., "Spectrum sensing for cognitive radio," Proceedings of the IEEE, vol. 97, no. 5, pp. 849-877, 2009.

He, H. and Jiang, H., "Deep learning based energy efficiency optimization for distributed cooperative spectrum sensing," IEEE Wireless Communications, 2019.

He, H. and Yan, J., "Cyber-physical attacks and defences in the smart grid: a survey," IET Cyber-Physical Systems: Theory \& Applications, vol. 1, no. 1, pp. 13-27, 2016. 
Jameel, F., Hamid, Z., Jabeen, F., Zeadally, S., and Javed, M. A., "A survey of device-to-device communications: Research issues and challenges," IEEE Communications Surveys $\&$ Tutorials, vol. 20, no. 3, pp. 2133-2168, 2018.

Jiang, H., He, H., Liu, L., and Yi, Y., "Q-learning for non-cooperative channel access game of cognitive radio networks," in 2018 International Joint Conference on Neural Networks (IJCNN). IEEE, 2018, pp. 1-7.

Jiang, N., Deng, Y., and Nallanathan, A., "Deep reinforcement learning for discrete and continuous massive access control optimization," in ICC 2020-2020 IEEE International Conference on Communications (ICC). IEEE, 2020, pp. 1-7.

Kaelbling, L. P., Littman, M. L., and Moore, A. W., "Reinforcement learning: A survey," Journal of artificial intelligence research, vol. 4, pp. 237-285, 1996.

Khalil, E., Dai, H., Zhang, Y., Dilkina, B., and Song, L., "Learning combinatorial optimization algorithms over graphs," in Advances in Neural Information Processing Systems, 2017, pp. 6348-6358.

Kingma, D. P. and Ba, J., "Adam: A method for stochastic optimization," arXiv preprint arXiv:1412.6980, 2014.

Kingma, D. P., Mohamed, S., Rezende, D. J., and Welling, M., "Semi-supervised learning with deep generative models," in Advances in neural information processing systems, 2014, pp. 3581-3589.

Kipf, T. N. and Welling, M., "Semi-supervised classification with graph convolutional networks," arXiv preprint arXiv:1609.0290\%, 2016.

Kiumarsi, B., Vamvoudakis, K. G., Modares, H., and Lewis, F. L., "Optimal and autonomous control using reinforcement learning: A survey," IEEE transactions on neural networks and learning systems, vol. 29, no. 6, pp. 2042-2062, 2017.

Krizhevsky, A., Sutskever, I., and Hinton, G. E., "Imagenet classification with deep convolutional neural networks," Advances in neural information processing systems, vol. 25, pp. 1097-1105, 2012.

Lee, D. H., Choi, K. W., Jeon, W. S., and Jeong, D. G., "Resource allocation scheme for device-to-device communication for maximizing spatial reuse," in 2013 IEEE Wireless Communications and Networking Conference (WCNC). IEEE, 2013, pp. 112-117.

Lee, N., Lin, X., Andrews, J. G., and Heath, R. W., "Power control for d2d underlaid cellular networks: Modeling, algorithms, and analysis," IEEE Journal on Selected Areas in Communications, vol. 33, no. 1, pp. 1-13, 2014. 
Li, H., "Multi-agent q-learning of channel selection in multi-user cognitive radio systems: A two by two case," in 2009 IEEE International Conference on Systems, Man and Cybernetics. IEEE, 2009, pp. 1893-1898.

Li, H., "Multi-agent q-learning for competitive spectrum access in cognitive radio systems," in 2010 Fifth IEEE Workshop on Networking Technologies for Software Defined Radio Networks (SDR). IEEE, 2010, pp. 1-6.

Li, Z., Yu, F. R., and Huang, M., "A distributed consensus-based cooperative spectrum-sensing scheme in cognitive radios," IEEE Transactions on Vehicular Technology, vol. 59, no. 1, pp. 383-393, 2009.

Lillicrap, T. P., Hunt, J. J., Pritzel, A., Heess, N., Erez, T., Tassa, Y., Silver, D., and Wierstra, D., "Continuous control with deep reinforcement learning," arXiv preprint arXiv:1509.02971, 2015.

Luo, C., Ji, J., Wang, Q., Chen, X., and Li, P., "Channel state information prediction for $5 \mathrm{~g}$ wireless communications: A deep learning approach," IEEE Transactions on Network Science and Engineering, 2018.

Luo, Z.-Q. and Zhang, S., "Dynamic spectrum management: Complexity and duality," IEEE journal of selected topics in signal processing, vol. 2, no. 1, pp. 57-73, 2008.

Ma, J., Cui, P., Kuang, K., Wang, X., and Zhu, W., "Disentangled graph convolutional networks," in International Conference on Machine Learning, 2019, pp. $4212-4221$.

Ma, J., Tang, W., Zhu, J., and Mei, Q., "A flexible generative framework for graph-based semi-supervised learning," in Advances in Neural Information Processing Systems, 2019, pp. 3276-3285.

Ma, J., Zhao, G., and Li, Y., "Soft combination and detection for cooperative spectrum sensing in cognitive radio networks," IEEE Transactions on Wireless Communications, vol. 7, no. 11, pp. 4502-4507, 2008.

Maaten, L. v. d. and Hinton, G., "Visualizing data using t-sne," Journal of machine learning research, vol. 9, no. Nov, pp. 2579-2605, 2008.

Meng, Y., Li, J.-D., Li, H.-Y., and Liu, P., "Graph-based user satisfaction-aware fair resource allocation in ofdma femtocell networks," IEEE Transactions on Vehicular Technology, vol. 64, no. 5, pp. 2165-2169, 2015.

Meng, Y., Li, J., Li, H., and Pan, M., "A transformed conflict graph-based resource-allocation scheme combining interference alignment in ofdma femtocell networks." IEEE Trans. Vehicular Technology, vol. 64, no. 10, pp. 47284737, 2015. 
Mitola, J., "Cognitive radio. an integrated agent architecture for software defined radio." 2002.

Mitola, J. and Maguire, G. Q., "Cognitive radio: making software radios more personal," IEEE personal communications, vol. 6, no. 4, pp. 13-18, 1999.

Mnih, V., Kavukcuoglu, K., Silver, D., Graves, A., Antonoglou, I., Wierstra, D., and Riedmiller, M., "Playing atari with deep reinforcement learning," arXiv preprint arXiv:1312.5602, 2013.

Mnih, V., Kavukcuoglu, K., Silver, D., Rusu, A. A., Veness, J., Bellemare, M. G., Graves, A., Riedmiller, M., Fidjeland, A. K., Ostrovski, G., et al., "Humanlevel control through deep reinforcement learning," nature, vol. 518, no. 7540, pp. 529-533, 2015.

Naparstek, O. and Cohen, K., "Deep multi-user reinforcement learning for distributed dynamic spectrum access," IEEE Transactions on Wireless Communications, vol. 18, no. 1, pp. 310-323, 2019.

Ng, Y. C., Colombo, N., and Silva, R., "Bayesian semi-supervised learning with graph gaussian processes," in Advances in Neural Information Processing Systems, 2018, pp. 1683-1694.

Nguyen, L. V., Nguyen, D. H., and Swindlehurs, A. L., "Svm-based channel estimation and data detection for massive mimo systems with one-bit adcs," in ICC 2020-2020 IEEE International Conference on Communications (ICC). IEEE, 2020, pp. 1-6.

Ni, Z., He, H., Zhao, D., Xu, X., and Prokhorov, D. V., "Grdhp: A general utility function representation for dual heuristic dynamic programming," IEEE transactions on neural networks and learning systems, vol. 26, no. 3, pp. 614627, 2014.

Nigam, K. and Ghani, R., "Analyzing the effectiveness and applicability of cotraining," in Proceedings of the ninth international conference on Information and knowledge management, 2000, pp. 86-93.

Pan, S., Hu, R., Fung, S.-f., Long, G., Jiang, J., and Zhang, C., "Learning graph embedding with adversarial training methods," IEEE transactions on cybernetics, 2019.

Peha, J. M., "Approaches to spectrum sharing," IEEE Communications magazine, vol. 43, no. 2, pp. 10-12, 2005.

Qu, M., Bengio, Y., and Tang, J., "Gmnn: Graph markov neural networks," arXiv preprint arXiv:1905.06214, 2019. 
Quan, Z., Cui, S., and Sayed, A. H., "Optimal linear cooperation for spectrum sensing in cognitive radio networks," IEEE Journal of selected topics in signal processing, vol. 2, no. 1, pp. 28-40, 2008.

Rost, P., Bernardos, C. J., De Domenico, A., Di Girolamo, M., Lalam, M., Maeder, A., Sabella, D., and Wübben, D., "Cloud technologies for flexible $5 \mathrm{~g}$ radio access networks," IEEE Communications Magazine, vol. 52, no. 5, pp. 68-76, 2014.

Rowley, H. A., Baluja, S., and Kanade, T., "Neural network-based face detection," IEEE Transactions on pattern analysis and machine intelligence, vol. 20, no. 1, pp. 23-38, 1998.

Sabeti, P., Farhang, A., Macaluso, I., Marchetti, N., and Doyle, L., "Blind channel estimation for massive mimo: A deep learning assisted approach," arXiv preprint arXiv:2002.10292, 2020.

Sakr, A. H., Tabassum, H., Hossain, E., and Kim, D. I., "Cognitive spectrum access in device-to-device-enabled cellular networks," IEEE Communications Magazine, vol. 53, no. 7, pp. 126-133, 2015.

Sen, P., Namata, G., Bilgic, M., Getoor, L., Galligher, B., and Eliassi-Rad, T., "Collective classification in network data," AI magazine, vol. 29, no. 3, pp. 93-93, 2008.

Silver, D., Schrittwieser, J., Simonyan, K., Antonoglou, I., Huang, A., Guez, A., Hubert, T., Baker, L., Lai, M., Bolton, A., et al., "Mastering the game of go without human knowledge," nature, vol. 550, no. 7676, pp. 354-359, 2017.

Srivastava, N., Hinton, G., Krizhevsky, A., Sutskever, I., and Salakhutdinov, R., "Dropout: a simple way to prevent neural networks from overfitting," The journal of machine learning research, vol. 15, no. 1, pp. 1929-1958, 2014.

Subhedar, M. and Birajdar, G., "Spectrum sensing techniques in cognitive radio networks: A survey," International Journal of Next-Generation Networks, vol. 3, no. 2, pp. 37-51, 2011.

Sutton, R. S. and Barto, A. G., Reinforcement learning: An introduction. MIT press, 2018.

Tan, L., Feng, Z., Li, W., Jing, Z., and Gulliver, T. A., "Graph coloring based spectrum allocation for femtocell downlink interference mitigation," in Wireless Communications and Networking Conference (WCNC), 2011 IEEE. IEEE, 2011, pp. 1248-1252.

Tehrani, M. N., Uysal, M., and Yanikomeroglu, H., "Device-to-device communication in 5g cellular networks: challenges, solutions, and future directions," IEEE Communications Magazine, vol. 52, no. 5, pp. 86-92, 2014. 
Tieleman, T. and Hinton, G., "Lecture 6.5-rmsprop: Divide the gradient by a running average of its recent magnitude," COURSERA: Neural networks for machine learning, vol. 4, no. 2, pp. 26-31, 2012.

Tsiropoulos, G. I., Yadav, A., Zeng, M., and Dobre, O. A., "Cooperation in 5g hetnets: Advanced spectrum access and d2d assisted communications," IEEE Wireless Communications, vol. 24, no. 5, pp. 110-117, 2017.

Uygungelen, S., Auer, G., and Bharucha, Z., "Graph-based dynamic frequency reuse in femtocell networks," in Vehicular Technology Conference (VTC Spring), 2011 IEEE 73rd. IEEE, 2011, pp. 1-6.

Vassiliades, V., Cleanthous, A., and Christodoulou, C., "Multiagent reinforcement learning: Spiking and nonspiking agents in the iterated prisoner's dilemma," IEEE transactions on neural networks, vol. 22, no. 4, pp. 639-653, 2011.

Veličković, P., Cucurull, G., Casanova, A., Romero, A., Lio, P., and Bengio, Y., "Graph attention networks," arXiv preprint arXiv:1710.10903, 2017.

Wang, B., Wu, Y., and Liu, K. R., "Game theory for cognitive radio networks: An overview," Computer networks, vol. 54, no. 14, pp. 2537-2561, 2010.

Weissensteiner, A., "A q-learning approach to derive optimal consumption and investment strategies," IEEE transactions on neural networks, vol. 20, no. 8, pp. 1234-1243, 2009.

Wu, F., Zhang, T., Souza Jr, A. H. d., Fifty, C., Yu, T., and Weinberger, K. Q., "Simplifying graph convolutional networks," arXiv preprint arXiv:1902.07153, 2019.

Xing, Y., Chandramouli, R., Mangold, S., et al., "Dynamic spectrum access in open spectrum wireless networks," IEEE journal on selected areas in communications, vol. 24, no. 3, pp. 626-637, 2006.

Xu, C., Cui, Z., Hong, X., Zhang, T., Yang, J., and Liu, W., "Graph inference learning for semi-supervised classification," arXiv preprint arXiv:2001.06137, 2020 .

Xu, Y., Wang, C., Chen, J., Wang, J., Xu, Y., Wu, Q., and Anpalagan, A., "Loadaware dynamic spectrum access for small-cell networks: a graphical game approach," IEEE Transactions on Vehicular Technology, vol. 65, no. 10, pp. 8794-8800, 2016.

Xu, Y., Wang, J., Wu, Q., Anpalagan, A., and Yao, Y.-D., "Opportunistic spectrum access in cognitive radio networks: Global optimization using local interaction games," IEEE Journal of Selected Topics in Signal Processing, vol. 6, no. 2, pp. 180-194, 2012. 
Xu, Y., Wang, J., Wu, Q., Zheng, J., Shen, L., and Anpalagan, A., "Dynamic spectrum access in time-varying environment: Distributed learning beyond expectation optimization," IEEE Transactions on Communications, vol. 65, no. 12 , pp. 5305-5318, 2017.

Xu, Y., Wu, Q., Shen, L., Wang, J., and Anpalagan, A., "Opportunistic spectrum access with spatial reuse: Graphical game and uncoupled learning solutions," IEEE Transactions on Wireless Communications, vol. 12, no. 10, pp. 48144826, 2013.

Yan, J., He, H., Zhong, X., and Tang, Y., "Q-learning-based vulnerability analysis of smart grid against sequential topology attacks," IEEE Transactions on Information Forensics and Security, vol. 12, no. 1, pp. 200-210, 2016.

Yang, C., Li, J., Semasinghe, P., Hossain, E., Perlaza, S. M., and Han, Z., "Distributed interference and energy-aware power control for ultra-dense d2d networks: A mean field game," IEEE Transactions on Wireless Communications, vol. 16, no. 2, pp. 1205-1217, 2016.

Yang, Z., Cohen, W. W., and Salakhutdinov, R., "Revisiting semi-supervised learning with graph embeddings," arXiv preprint arXiv:1603.08861, 2016.

Yin, R., Yu, G., Zhang, H., Zhang, Z., and Li, G. Y., "Pricing-based interference coordination for d2d communications in cellular networks," IEEE Transactions on Wireless Communications, vol. 14, no. 3, pp. 1519-1532, 2014.

Yu, C., Zhang, M., Ren, F., and Tan, G., "Emotional multiagent reinforcement learning in spatial social dilemmas," IEEE transactions on neural networks and learning systems, vol. 26, no. 12, pp. 3083-3096, 2015.

Yucek, T. and Arslan, H., "A survey of spectrum sensing algorithms for cognitive radio applications," IEEE communications surveys $\&$ tutorials, vol. 11, no. 1, pp. 116-130, 2009.

Zhang, G., Hu, J., Heng, W., Li, X., and Wang, G., "Distributed power control for d2d communications underlaying cellular network using stackelberg game," in 2017 IEEE Wireless Communications and Networking Conference (WCNC). IEEE, 2017, pp. 1-6.

Zhang, L., Jiang, T., and Luo, K., "Dynamic spectrum allocation for the downlink of ofdma-based hybrid-access cognitive femtocell networks," IEEE Transactions on Vehicular Technology, vol. 65, no. 3, pp. 1772-1781, 2016.

Zhang, W., Guo, Y., Liu, H., Chen, Y., Wang, Z., and Mitola III, J., "Distributed consensus-based weight design for cooperative spectrum sensing," IEEE Transactions on Parallel and Distributed Systems, vol. 26, no. 1, pp. 54-64, 2014. 
Zhang, Y., Pal, S., Coates, M., and Ustebay, D., "Bayesian graph convolutional neural networks for semi-supervised classification," in Proceedings of the AAAI Conference on Artificial Intelligence, vol. 33, 2019, pp. 5829-5836.

Zhang, Y. and Wang, S., "Resource allocation for cognitive radio-enabled femtocell networks with imperfect spectrum sensing and channel uncertainty," IEEE Transactions on Vehicular Technology, vol. 65, no. 9, pp. 7719-7728, 2016.

Zhang, Z., Cui, P., and Zhu, W., "Deep learning on graphs: A survey," arXiv preprint arXiv:1812.04202, 2018.

Zhao, Q. and Swami, A., "A survey of dynamic spectrum access: Signal processing and networking perspectives," in Acoustics, speech and signal processing, $200 \%$. ICASSP 200\%. IEEE international conference on, vol. 4. IEEE, 2007, pp. IV-1349.

Zhou, D., Bousquet, O., Lal, T. N., Weston, J., and Schölkopf, B., "Learning with local and global consistency," in Advances in neural information processing systems, 2004, pp. 321-328.

Zhou, X., Shen, F., Liu, L., Liu, W., Nie, L., Yang, Y., and Shen, H. T., "Graph convolutional network hashing," IEEE transactions on cybernetics, 2018.

Zhu, X., Ghahramani, Z., and Lafferty, J. D., "Semi-supervised learning using gaussian fields and harmonic functions," in Proceedings of the 20th International conference on Machine learning (ICML-03), 2003, pp. 912-919. 